\title{
Exploiting Reuse and Vectorization in Blocked Stencil Computations on CPUs and GPUs
}

\author{
Tuowen Zhao \\ School of Computing \\ University of Utah \\ ztuowen@cs.utah.edu
}

\author{
Protonu Basu \\ Facebook \\ protonu@fb.com
}

\author{
Samuel Williams \\ Computational Research Division \\ Lawrence Berkeley National Lab \\ swwilliams@lbl.gov
}

\author{
Mary Hall \\ School of Computing \\ University of Utah \\ mhall@cs.utah.edu
}

\author{
Hans Johansen \\ Computational Research Division \\ Lawrence Berkeley National Lab \\ hjohansen@lbl.gov
}

\begin{abstract}
Stencil computations in real-world scientific applications may contain multiple interrelated stencils, have multiple input grids, and use higher order discretizations with high arithmetic intensity and complex expression structures. In combination, these properties place immense demands on the memory hierarchy that limit performance. Blocking techniques like tiling are used to exploit reuse in caches. Additional fine-grain data blocking can also reduce TLB, hardware prefetch, and cache pressure.

In this paper, we present a code generation approach designed to further improve tiled stencil performance by exploiting reuse within the block, increasing instruction-level parallelism, and exposing opportunities for the backend compiler to eliminate redundant computation. It also enables efficient vector code generation for CPUs and GPUs. For a wide range of complex stencil computations, we are able to achieve substantial speedups over tiled baselines for the Intel KNL, Intel Skylake-X, and NVIDIA P100 architectures.
\end{abstract}

\section{CCS CONCEPTS}

- Software and its engineering $\rightarrow$ Source code generation; • Computing methodologies $\rightarrow$ Parallel programming languages.

\section{KEYWORDS}

Compiler Optimization, Stencil, vectorization

\section{ACM Reference Format:}

Tuowen Zhao, Protonu Basu, Samuel Williams, Mary Hall, and Hans Johansen. 2019. Exploiting Reuse and Vectorization in Blocked Stencil Computations on CPUs and GPUs. In The International Conference for High Performance Computing, Networking, Storage, and Analysis (SC '19), November 17-22, 2019, Denver, CO, USA. ACM, New York, NY, USA, 13 pages. https://doi.org/10.1145/3295500.3356210

Permission to make digital or hard copies of all or part of this work for personal or classroom use is granted without fee provided that copies are not made or distributed for profit or commercial advantage and that copies bear this notice and the full citation on the first page. Copyrights for components of this work owned by others than the author(s) must be honored. Abstracting with credit is permitted. To copy otherwise, or republish, to post on servers or to redistribute to lists, requires prior specific permission and/or a fee. Request permissions from permissions@acm.org.

SC '19, November 17-22, 2019, Denver, CO, USA

(c) 2019 Copyright held by the owner/author(s). Publication rights licensed to ACM. ACM ISBN 978-1-4503-6229-0/19/11 ..\$15.00

https://doi.org/10.1145/3295500.3356210

\section{INTRODUCTION}

Stencil computations are ubiquitous in scientific applications that solve partial differential equations using the finite difference or finite volume methods, where the derivative at each point in space is calculated as a weighted sum of neighboring point values (a "stencil"). A stencil's order of accuracy is the exponent on the relationship between grid spacing (array size) and error - both small grid spacings (large arrays) and high order can result in low error. A stencil's order greatly impacts the optimizations needed to achieve high performance. Low-order discretizations result in smaller stencils that have limited data reuse, are typically bound by memory bandwidth, and thus underutilize the compute capability afforded by manycore, wide vector, and GPU architectures. Much of the prior work in this field has been based on lower order stencils and has thus focused on techniques to reduce main memory data movement [6, 13, 14, 20, 21, 23, 28, 33, 36, 38, 41, 45].

As processor architectures become more compute-intensive [37], computational scientists are increasingly turning to high-order schemes that perform more computation per point (more computeintensive) but can attain equal error with larger grid spacings (smaller arrays). Although higher-order stencils inherently result in higher arithmetic intensity, they also place immense pressure on register file, cache, TLB, and hardware prefetchers. Worse still, to further utilize available compute capability, stencil computations are often a composition of multiple high-order stencils, such as the $8^{t h}$-order hypterm kernel, described in [12], and depicted in Figure 1. It computes five stencils that operate on eight input fields.

Prior work on optimizing high-order stencils leverages the associativity of the weighted sums in a stencil computation; such operations can therefore be safely reordered to achieve the same result within round-off tolerances. Consequently, execution order can be optimized to exploit data reuse, and thus reduce memory load/store operations and reduce register pressure [3, 10, 24, 25, 29]. Prior associative reordering methods for stencils are limited in several ways. Most focus on reuse of individual data elements, with an eye towards optimizing scalar registers [24, 25]. Where reuse of vectors is considered to support vector code generation, it is limited to isotropic, constant-coefficient stencils [3], or arises from a post-pass vectorization, preceded by DLT (data-layout transformation) optimization [29]. In some cases, cross-iteration reuse is identified as a byproduct of loop unrolling $[10,25]$. Only one of 
these approaches targets GPUs, and it exploits reuse just within an expression [24].

This paper addresses these limitations, describing a vector code generator for general stencil computations targeting both CPUs and GPUs. It identifies data reuse without unrolling within a finegrain block of a stencil computation. For further optimization gains, this approach to reuse analysis and vectorization can also work in tandem with a fine-grained blocked data layout that decomposes the original grid domain into small, fixed-size multi-dimensional subdomains [2, 17, 40], such as bricks [44], which have been shown to achieve performance portability across CPU and GPU. Bricks are stored contiguously in memory to enable a number of optimizations. First, accesses within a brick are part of a single address stream, mitigating the negative impact of blocking on hardware prefetchers and TLB. Second, when combined with vector folding [39], an individual dimension can be smaller than the vector width; this flexibility can reduce cache and register pressure for complex stencils like hypterm. Other stencil optimizations such as temporal blocking and wavefront parallelism are beyond the scope of this paper, but are complementary and can be combined with our method.

This paper makes the following contributions: (1) it presents a vector code generation algorithm for general stencil computations that exploits data reuse within a block without unrolling, and targets both CPUs and GPUs; (2) it compares the effectiveness of the code generation approach for iteration space tiling vs. bricks on CPUs, isolating the benefits of each; (3) it offers the first description of node-level vector code generation for bricks; (4) it presents performance results on 24 stencils, including real-world proxy stencils such as hypterm, demonstrating performance gains on Intel Knights Landing (Xeon Phi) processors (up to $3.4 \times$ ), Intel Xeon Skylake-X (1.3×), and NVIDIA P100 (1.6×).

\section{BACKGROUND AND MOTIVATION}

In this section, we motivate our approach, using the hypterm kernel, with code and the compiler's expression tree shown in Figure 1. Stencils like hypterm exhibit high temporal reuse across stencil iterations, e.g., cons $[i m x][k][j][i+1]$ and cons $[i m x][k][j][i-1]$ two iterations later. It is common to use tiling to exploit this reuse in caches or unrolling/unroll-and-jam to enable optimizations for reuse in registers. Additional array common subexpressions within and across expressions, such as the results of the shaded operators at the bottom of Figure 1, can also be reused in registers. Due to the complexity of hypterm, exploiting such register reuse can lead to severe register pressure; exposing cross-iteration reuse using unrolling may increase register pressure, and even cause instruction cache misses. In addition, hypterm has high arithmetic intensity, with 358 floating-point operations per iteration. Achieving high performance also demands efficient use of wide SIMD units in CPUs and SIMT threads in GPUs.

Another consideration is that hypterm places immense pressure on the TLB and hardware prefetcher due to the number of independent data streams. One $k$-j plane of hypterm requires 133 simultaneously active read or write data streams (corresponding to different registers, cache lines and potentially, TLB entries). Tiling will exacerbate this problem. To reduce the number of data streams for such stencils, prior work has developed variations of blocked data layouts, where the original grid domain is decomposed into small, fixed-sized multi-dimensional subdomains [2, 17, 40]. In this paper, we expand on the concept of bricks, where these subdomains are stored contiguously in memory [44]. Using an $8 \times 8 \times 8$ brick size and stencil radius $\leq 8$, we access the elements within a brick using a single stream as opposed to 64 streams for a tiled code. The computation inside one brick would be similar to an $8 \times 8 \times 8$ tiled stencil.

Taken together, this paper describes a vector code generator that can balance the aforementioned optimization requirements of high-order stencils such as hypterm. Our approach exploits reuse within a multi-dimensional data block, arising from either tiling, which reorders the computation, or bricks, which also reorganizes the data layout. As stencils are known to pose challenges to vectorization due to issues of alignment [15], the approach must expose aligned vector operations. Additionally, our approach further reduces arithmetic intensity by exposing opportunities for array common subexpression elimination [10]. The remainder of this section provides the foundation for the code generation approach.

\subsection{Stencils as Gather or Scatter Operations}

The kernel of a stencil computation typically contains a weighted sum of neighboring points. Such sums are most commonly expressed as gather operations, as in the 5-point 2D stencil code of Figure 2(a), where the value of this sum is calculated for each iteration of a loop nest by gathering its neighboring inputs (some of which are widely spaced in memory), individually weighting them, and summing them. Figure 3(a) visualizes this gather computational pattern for the 5-point stencil code.

However, one can observe that these weighted sums are associative and can be reordered without changing the meaning of the computation. This concept is associative reordering. Therefore, an alternative implementation of the 5-point stencil is a scatter operation, where one input is weighted and scattered to all the neighboring points that use it as a term in the sum. Figure 3(b) shows the resultant scatter pattern for the 5-point stencil. Scatters have several advantages including minimizing the number of loads, and improving instruction level parallelism, but may increase the number of stores. For high-order stencils that access a large number of inputs to compute each output point, an approach that favors reducing loads is preferable to one that reduces stores. We also find that the output data often resides in registers, particularly on GPUs, or in L1 cache, so store cost is typically low. Scatter also matches the strengths and weaknesses of bricks. Loads are more costly, because accesses that cross brick boundaries introduce indexing overhead due to the adjacency list, and unaligned loads are not applicable.

In the following, we will select some portions of the computation with high reuse to be computed using scatter.

\subsection{Overview of Approach}

The current code generator uses a domain-specific frontend, implemented in Python, that accepts stencil descriptions as input, shown in Figure 2(c). The output of the code generator is integrated into $\mathrm{C}$ code as in Figure 2(d). From this specification, we can generate either tiled or brick code that incorporates scatter, as used in the experiments of Section 5. The data layout for the tiled code is a 


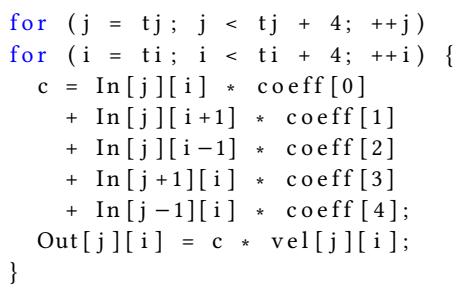

(a) Stencil in a tiled region.

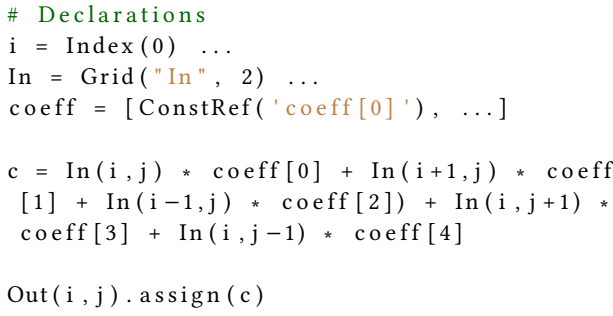

(c) Input to the code generator: kernel. py

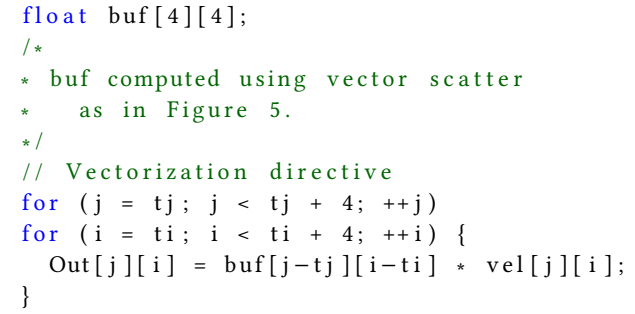

(b) Split stencil with buffer to expose reuse.

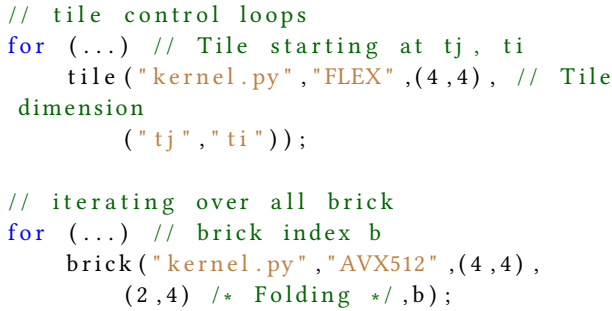

(d) Adding kernel to $\mathrm{C}$ code.

Figure 2: A 5-point stencil example.

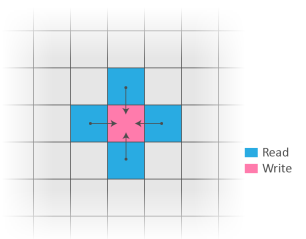

(a) Gather

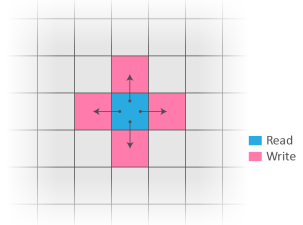

(b) Scatter
Figure 3: Gather vs. scatter operations for 5 points in $2 D$.

\section{REUSE-BASED EXPRESSION SPLITTING}

This section describes how to split a stencil kernel into compute stages based on its reuse pattern. We first build an expression directed acyclic graph (DAG) from the code. For simplicity, we illustrate the algorithm using the running example of Figure 2 whose expression DAG is shown in Figure 4, but also refer to the DAG for hypterm in Figure 1 when discussing operator grouping. The code generation framework obtains this graph by identifying the assignments to grids in the original abstract syntax tree (AST), and the operators, constants and grid references on the right hand side. The output grid is the target of the DAG, and the operand grids are annotated with their name and offset from the iterator. In Figure 2, with iterator $[j, i]$, reference $\operatorname{In}[j][i-1]$ is represented by node In: $0,-1$. Neighboring associative operators of the same type are combined to a single operator with three or more terms, resulting in the 5-way addition in Figure 4. Observe that such DAGs can represent a broad variety of stencil codes: variable coefficients, multiple stencils, and several inputs multiplied by the same coefficient.

In this phase we start from the expression DAG using two major steps (1) identify reuse profitability and select associative operators to be reordered by postorder traversal of the expression DAG; (2) identify opportunities to group operators across expressions into stages to further improve data reuse.

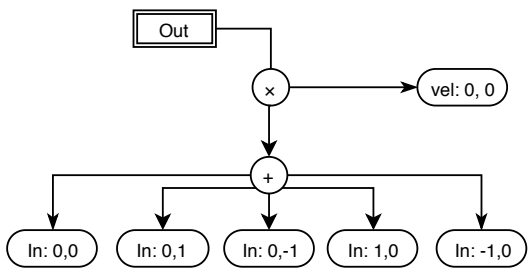

Figure 4: Expression DAG for the 5-point stencil of Figure 2.

\subsection{Reuse Profitability in Operators}

The first step of the code generation algorithm is to mark associative operators in the expression DAG for reordering based on a profitability analysis. We define the profitability of scatter using the reduction of the number of inputs that are simultaneously live for each iteration step. We calculate profitability using a postorder traversal of the expression DAG, and we then mark the operators to be reordered that exceed a profitability threshold.

The postorder traversal collects $R(E)$, grids and offsets from the DAG for expression $E$, as in Equation 1. The number of elements in this set is then the number of unique reads when calculating this subexpression as how they appear in the expression DAG.

$$
R(E)=\{\langle g, \vec{o}\rangle \mid \text { grid } g \text { appears in } E \text { with offset } \vec{o}\}
$$

When $E$ is an associative operator, it consists of multiple terms (subexpressions), $E_{i}$. When we shift the terms of the associative operator between iterations we are effectively adding a per-term constant $\vec{\delta}_{i}$ to the offset of the corresponding term. This shift, $\vec{\delta}_{i}$, is sometimes referred to as the retiming vector in loop shifting [29]. If we add the shifts to all terms of the operator then we can collect the set of grids and offsets with Equation 2. The number of elements in this set is then the number of unique reads when calculating the associative operator with each term shifted by $\Delta=\left\{\vec{\delta}_{i}\right\}$. 


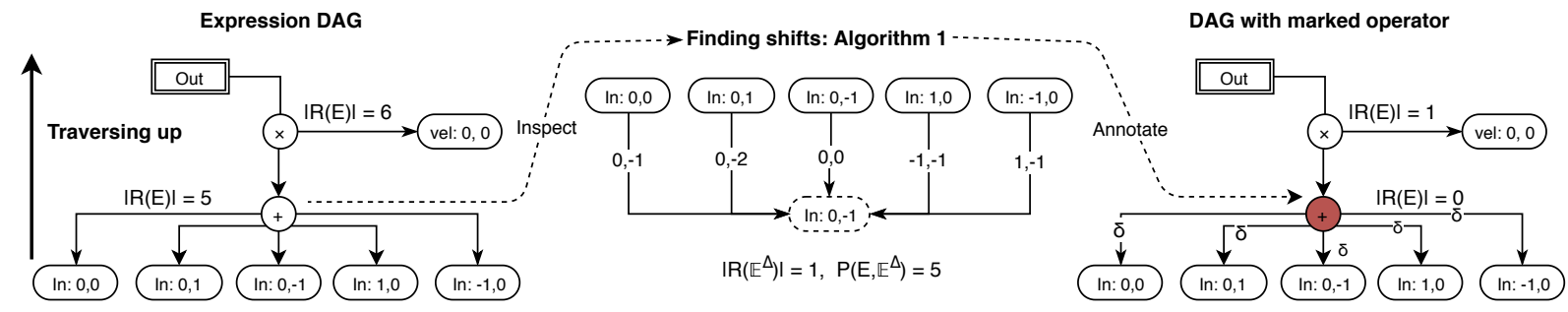

Figure 5: Illustration of profitability analysis and operator marking for the 5-point stencil in Figure 2. During DAG traversal (dashed arrow), Algorithm 1 is used to try to minimize distinct references by adding shifts to each term (numbers on edge). If the operator should be reordered (per Equation 3), the DAG is marked with shift $\delta_{i}$.

$$
R\left(\mathbb{E}^{\Delta}\right)=\bigcup_{i}\left\{\left\langle g, \vec{o}+\vec{\delta}_{i}\right\rangle \mid\langle g, \vec{o}\rangle \in R\left(E_{i}\right)\right\}
$$

Using the cardinality of the two sets from Equation 1 and Equation 2, we can evaluate the profitability $P$ of a reordered expression $E^{\Delta}$ as in Equation 3. Reordering is marked as profitable if reads are reduced by a large fraction; that is, when $P$ exceeds a global threshold $t_{1} \geq 1$.

$$
P\left(E, \mathbb{E}^{\Delta}\right)=\frac{|R(E)|}{\left|R\left(\mathbb{E}^{\Delta}\right)\right|} \geq t_{1}
$$

Note that $|R(E)|$ is a property of the stencil computation. In order to maximize $P$, we only need to find a set of shifts, $\Delta$, that minimize $\left|R\left(\mathbb{E}^{\Delta}\right)\right|$

This process is illustrated in Figure 5 for the 5 -point stencil. During post-order traversal, when an associative operator is encountered, Algorithm 1 is used to inspect its terms; this is denoted in the figure by a dashed arrow. We note that in the original computation there are five distinct references, thus $|R(E)|=5$. The algorithm assigns each term with one shift value that is marked on the edges; with shift this produces the same reference: In: $0,-1$. Thus, from Equation 2, the number of distinct references after the shift is 1 . This gives us a reuse profitability of 5 from Equation 3 . Assuming this profitability is above the predefined threshold $t_{1}$, this addition is marked in the original graph with the shift values produced from Algorithm 1, $\delta$, annotated to edges leading to each term.

The number of possible shift amounts for one term is related to the radius of the stencil; the total search space is exponential in the number of terms. This search space is potentially too large to search exhaustively. However, the set of offsets that minimizes $P\left(E^{\Delta}\right)$ has the properties of Equation 4: the minimum only arises when for each term, it either has no common grid input with any other terms, or it has at least one read that can be reused after shifting.

$$
\begin{aligned}
& \forall E_{i}, \text { either } \\
& \qquad\left\{\begin{array}{l}
\forall\langle g, \vec{o}\rangle \in R\left(E_{i}\right), g \notin R\left(\mathbb{E}-\left\{E_{i}\right\}\right), \text { or } \\
\exists\langle g, \vec{o}\rangle \in R\left(E_{i}\right),\left\langle g, \vec{o}+\vec{\delta}_{i}\right\rangle \in R\left(\left(\mathbb{E}-\left\{E_{i}\right\}\right)^{\Delta-\left\{\delta_{i}\right\}}\right)
\end{array}\right.
\end{aligned}
$$

Proof. Equation 4 can be proven using contradiction by assuming the negation: $\left|R\left(\mathbb{E}^{\Delta^{*}}\right)\right|$ is the minimum but has an $E_{i}$ that shares a common grid input and does not have reuse with shift $\delta_{i}$. In this case, we can change $\delta_{i}$ so that at least one read is reused with some other term. We have the new $\left|R\left(\mathbb{E}^{\Delta^{\prime}}\right)\right|$, which will be smaller by at least one. It contradicts the assumption that $\Delta^{*}$ gives the minimum. Thus, Equation 4 must be true.

Based on (4), we developed a fast greedy algorithm in Algorithm 1. The complexity of this algorithm is $O\left(n^{4} \log n\right)$ where $n=\sum_{i}\left|R\left(E_{i}\right)\right|$. This $n$ is bounded by the number of reads in the original stencil code, $N$. In fact, this is only a loose upper bound for the complexity, and for associative operators that only have one offset for all reads in one term, such as the hypterm stencil in Figure 1 and many of other stencils, its complexity is only $O(n)$. After the scatter is decided, we can then compute the profitability and mark associative operators for grouping.

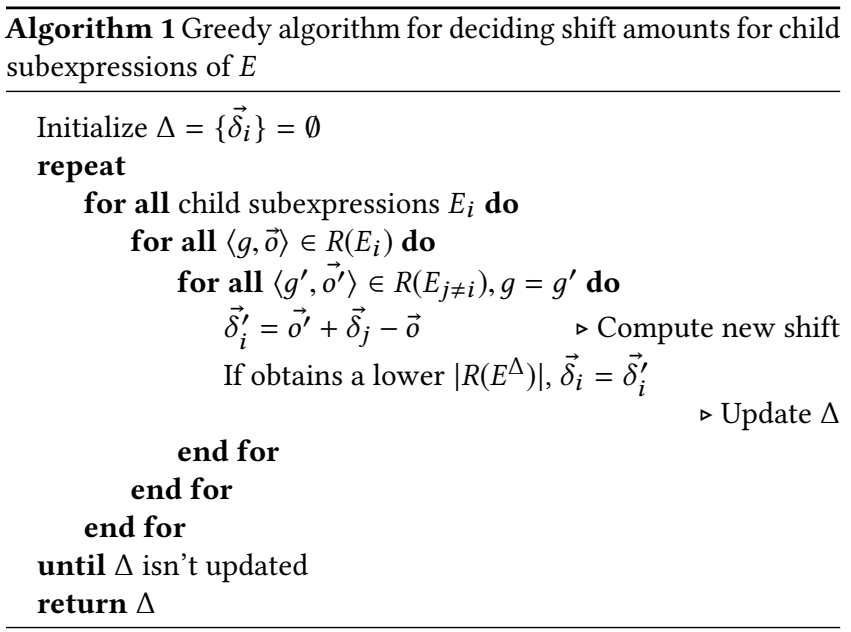

\subsection{Cross-operator Reuse}

Step 2 in our framework will enable optimization of loads across parts of the expression DAG by attempting to group reordered operators utilizing the same data as input to be computed together. Each marked computation can be computed once all the values from its subexpression are available, which includes all subexpressions that are marked. This creates a dependence graph that includes all marked subexpressions where edges are contracted from the original expression DAG.

Beyond optimizing for reuse across subexpressions, operator grouping also impacts the number of buffers that are simultaneously 


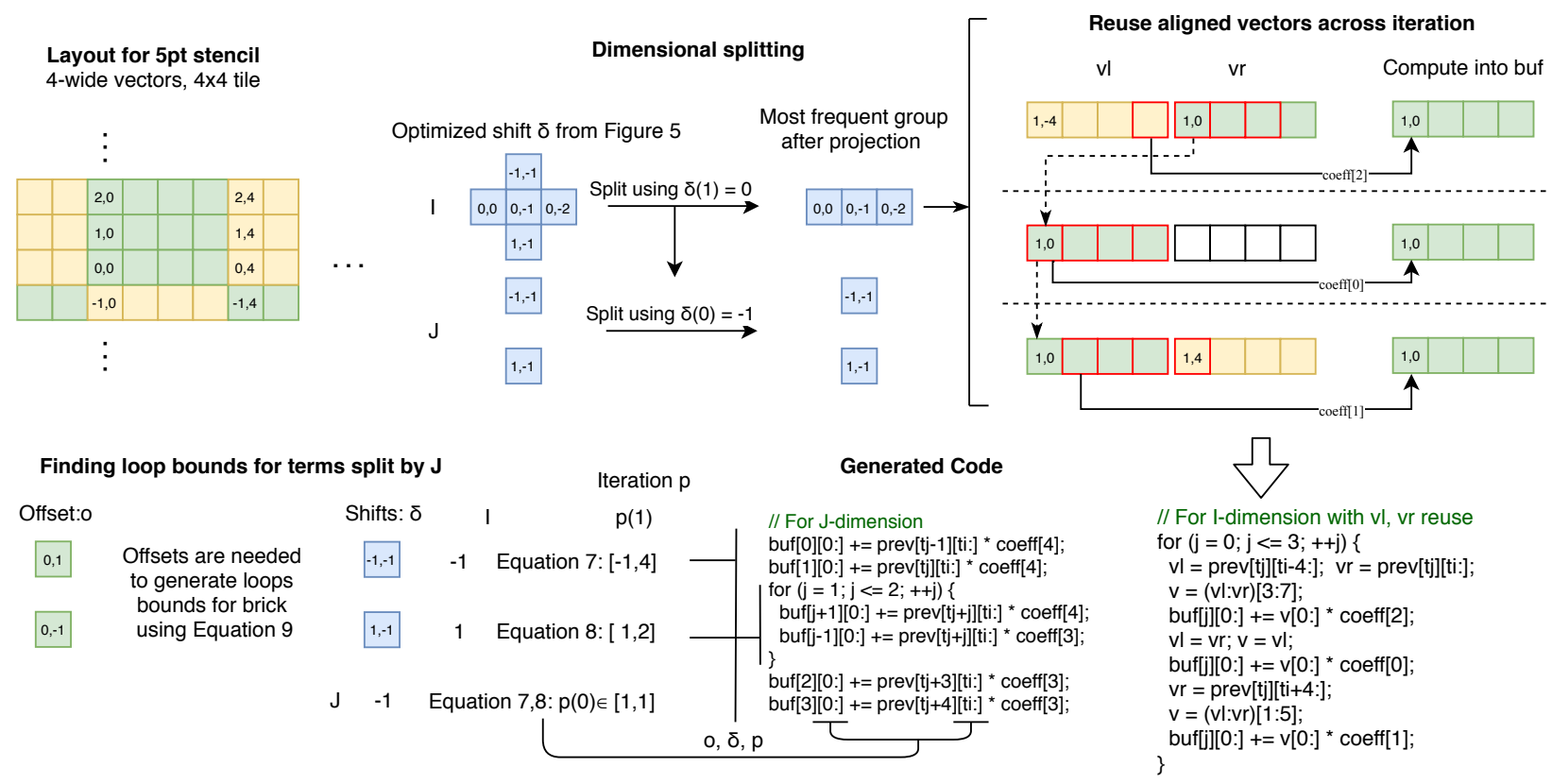

Figure 6: Vector code generation for 5-point stencil example in Figure 2,5. The generated code employs dimensional splitting and reuses aligned vector load.

live. We use a modified topological sort based on a priority tuple measure similar to the one used in [25] to break ties during the sort when multiple alternatives are present. Applying this process results in a linearized sequence that tries to balance buffer usage and the number of operators that can be computed concurrently. This step has a complexity of at most $O\left(\mathrm{~m}^{2}\right)$ where $m$ is the number of marked subexpressions.

We can then scan the sequence and merge nearby operators into one stage based on a measure of hardware pressure, which is derived from (1) the number of distinct inputs; and, (2) the number of buffers accessed. Both of them can represent the grouped operators' pressure on the registers. The first value can be computed using Algorithm 1. To determine viability, we compute a weighted sum of the two measures after the grouping and compare against a global threshold $t_{2}$ representing the architectural constraint, as in Equation 5, where $\mathbb{E}$ represents the group of operators' expressions and $|B|$ is the number of buffers in the current group. Merging can be attempted at most twice for each buffer. This results in a similar complexity of Algorithm 1 where $n=N$, the number of reads in the original stencil code. Since $N>1, m<N$, we have a total complexity of $O\left(N^{4} \log N\right)$. As noted previously, for stencils whose associative operator has only one offset for each term, this complexity reduces to $O\left(m^{2}+N\right)$.

$$
\left|R\left(\mathbb{E}^{\Delta}\right)\right|+k|B| \leq t_{2}
$$

Consider the example in Figure 1. Our approach recognizes that there is potential for reuse within the group of associative operators colored by blue. Note that adding the blue operator on the left to a group consisting of the blue operator on the right will not affect the first term of Equation 5 . The equation only approaches the threshold by the extra buffer. However, when adding the associative operator colored red to this group, both terms increase and the threshold is reached faster, preventing aggressive grouping.

\section{VECTOR CODE GENERATION}

Once all compute stages are created, we obtain a sequence of groups of operators in the expression DAG. For any groups not identified by the algorithm as profitable for reordering, a gather, using the original computation, will be generated. Other stages contain associative operators that are profitable for reordering; these operators will have a shift, $\vec{\delta}_{i}$, associated with each term, $\left\{E_{i}\right\}$, of the operator. We can then generate code for each stage in the sequence using either gather or scatter. In this section, we describe how vector code is generated from $\left\{E_{i}\right\}$ and $\Delta$ with loops. We also present additional optimizations that we used. A walk through of the code generation is shown in Figure 6 for the 5-point stencil.

The techniques in this section are based on scanning the iteration space and the expression DAG side-by-side, which results in a complexity of $O(|S||V|)$, where $|S|$ is the size of the tile and $|V|$ is the number of nodes in the expression DAG.

\subsection{Vector Scatter}

While we focus on scattered computation with vectors, this is derived from reordering using scalar values. We first use the shifts computed for each term. For iteration $\vec{p}$ within the tile, we can determine the shifted loop index, the source of the scatter, by subtracting $\vec{\delta}_{i}$ as in Equation 6. Each of the sources will scatter the corresponding subexpression that is shifted by adding $\vec{\delta}_{i}$ to all the original array indices. The scatter can then walk through all such $\overrightarrow{p^{\prime}}$ 
and compute the value for each term to the destination with $\overrightarrow{p^{\prime}}+\vec{\delta}_{i}$.

$$
\overrightarrow{p^{\prime}}=\vec{p}-\vec{\delta}_{i}
$$

When generating vector code, we add restrictions to the destination so that only destinations that are aligned are written. To accommodate wide vectors and small data blocking, we assume the vector is a multidimensional vector-sized rectangle, that exhibits a length $v(d)$ on each tile dimension $d$. Then we have an aligned destination whose offset within the tile is a multiple of the vector length on all dimensions. With this reduced set of destinations, the meaning of the calculation is preserved since every location in the block is in one of the aligned vectors.

We notice that we can generate some loops to reduce the code size, since $\vec{\delta}_{i}$ reflects the amount of loop shifting applied to the term. $T(d)$ represents the tile size on dimension $d$. We can formulate the loop bounds as in Equations 7-9. Equation 7 represents the total range of the shifted iteration space. All or part of this range can use loops while some iterations might need to be peeled from either side of this range.

$$
\left\{\begin{array}{l}
0-\max _{i}\left(\delta_{i}(d)\right) \\
T(d)-v(d)-\min _{i}\left(\delta_{i}(d)\right)
\end{array}\right.
$$

Equation 8 represents when the writes for all terms are inside the original tiled space. A loop can be generated on the tiled code for all iterations in this range.

$$
\left\{\begin{array}{l}
0-\min _{i}\left(\delta_{i}(d)\right), \\
T(d)-v(d)-\max _{i}\left(\delta_{i}(d)\right)
\end{array}\right.
$$

Equation 9 represents when all reads are inside the blocked data region. For bricks, a loop can be generated by combining Equation 8 and Equation 9.

$$
\left\{\begin{array}{l}
0-\min _{i}\left(o_{i}(d)+\delta_{i}(d)\right), \\
T(d)-v(d)-\max _{i}\left(o_{i}(d)+\delta_{i}(d)\right)
\end{array}\right.
$$

The lower left of Figure 6 shows how loop bounds are inferred for two of the terms that are split from the original stencil using Equations 7 and 8.

\subsection{Optimizations}

We employ two additional optimizations when generating scatter for one stage, (1) further data reuse within vector registers using aligned vector load and vector align instructions; and, (2) dimensional splitting which divides the stencil expression and calculates it along one dimension at a time [18] to further reduce peeling. These optimizations are illustrated in the upper half of Figure 6.

We noticed that more reuse can be exploited with an aligned vector load. This optimization is especially useful for bricks where a logical vector may cross the brick boundary and must be merged using vector aligns. This merging, using alignr intrinsics on the $\mathrm{CPU}$, is happening for almost every iteration when we are traversing the contiguous direction while the two aligned vectors will be the same for several iterations. These aligned loads can then be cached and reused for consecutive iterations. Upper right of Figure 6 shows that we can create two temporary vectors $v l$ and $v r$ to cache the aligned vectors. With this optimization, we only load these values three times, $2+0+1$, instead of five, $2+1+2$.
We also employ dimensional splitting of the stencil to further reduce the final code size. We observe that Equation 8 is only related to the shift selected for each term, and it is often more constrained than Equation 9. If we directly generate code for the 5-point stencil example in Figure 6, the calculation for terms on the I-dimension is unnecessarily peeled because of the two terms on the J-dimension. We can then group the terms based on their values to increase the range of Equation 8. We achieve this by picking one dimension at a time and group the terms based on their shifts on the other dimensions. We then select the most frequent groups to be computed together. Larger loops can be created for the other dimensions where they have a common shift value. We repeat this process for each of the dimensions to fully split the stencils. This process is applied to the 5-point stencil in Figure 6. For stencils such as CNS, Figure 1, we can achieve a perfect split as peeling only happens for one dimension at a time. Without dimensional splitting, no loops are possible for the stencil with $8 \times 8 \times 8$ tile.

\subsection{Vectorizing on GPU}

As observed by prior work [46], GPUs offer the same vector merging capability as alignr intrinsics on the CPU using either shared memory or shuffle instructions, __shfl_up and __shfl_down. This allows us to transfer our vectorization method onto NVIDIA GPUs and use each warp as one vector that has a length of 32. Also, through use of vector folding and combinations of multiple shuffles we enable support for smaller data blocks such as $8 \times 8 \times 8$ or $4 \times 4 \times 4$.

\section{EXPERIMENTAL RESULTS}

This section presents performance results for the generated code for both CPUs and GPUs, applied to tiled or brick code.

\subsection{Target Architectures}

Intel Knights Landing. The Intel Xeon Phi 7250 Knights Landing (KNL) has 68 physical cores organized into a 2D on-chip mesh of 34 tiles each with two CPU cores $^{1}$ and a shared $1 \mathrm{MB}$ L2 cache. Each core has a private $32 \mathrm{~KB} \mathrm{L1}$ data cache, implements 4-way multithreading, and has two AVX-512 vector processing units (VPUs). AVX-512 instructions operate on 8 double-precision or 16 singleprecision floating-point data elements in a SIMD fashion. Its theoretical peak performance is $2611.2 \mathrm{GFLOP} / \mathrm{s}$ double-precision fused multiply-and-add. Each chip has both standard DDR4 DRAM memory and high-bandwidth MCDRAM memory that we configured as a last level cache using the quadcache mode, which yields a peak STREAM performance of $332 \mathrm{~GB} / \mathrm{s}$.

Intel Xeon Gold (Skylake-X). The Intel Xeon Gold $6130 \mathrm{CPU}$ has 16 physical cores. Each core has a private $32 \mathrm{~KB}$ L1 data cache and $1 \mathrm{MB}$ L2 cache, implements 4-way multithreading, and has two AVX-512 vector processing units (VPUs). Each core has a nominal frequency of $2.1 \mathrm{GHz}$. The whole CPU has a theoretical peak performance is $1075.2 \mathrm{GFLOP} / \mathrm{s}$. Concurrently, 6 DDR4 memory controllers provide a STREAM bandwidth of $85 \mathrm{~GB} / \mathrm{s}$.

NVIDIA P100. The P100 GPU has 56 streaming multiprocessors. Each streaming multiprocessor has 64 single-precision and 32 double-precision CUDA cores and has a warp size of 32. Each

\footnotetext{
${ }^{1}$ We use 32 tiles for a total of 64 cores in all our experiments to isolate system overhead.
} 

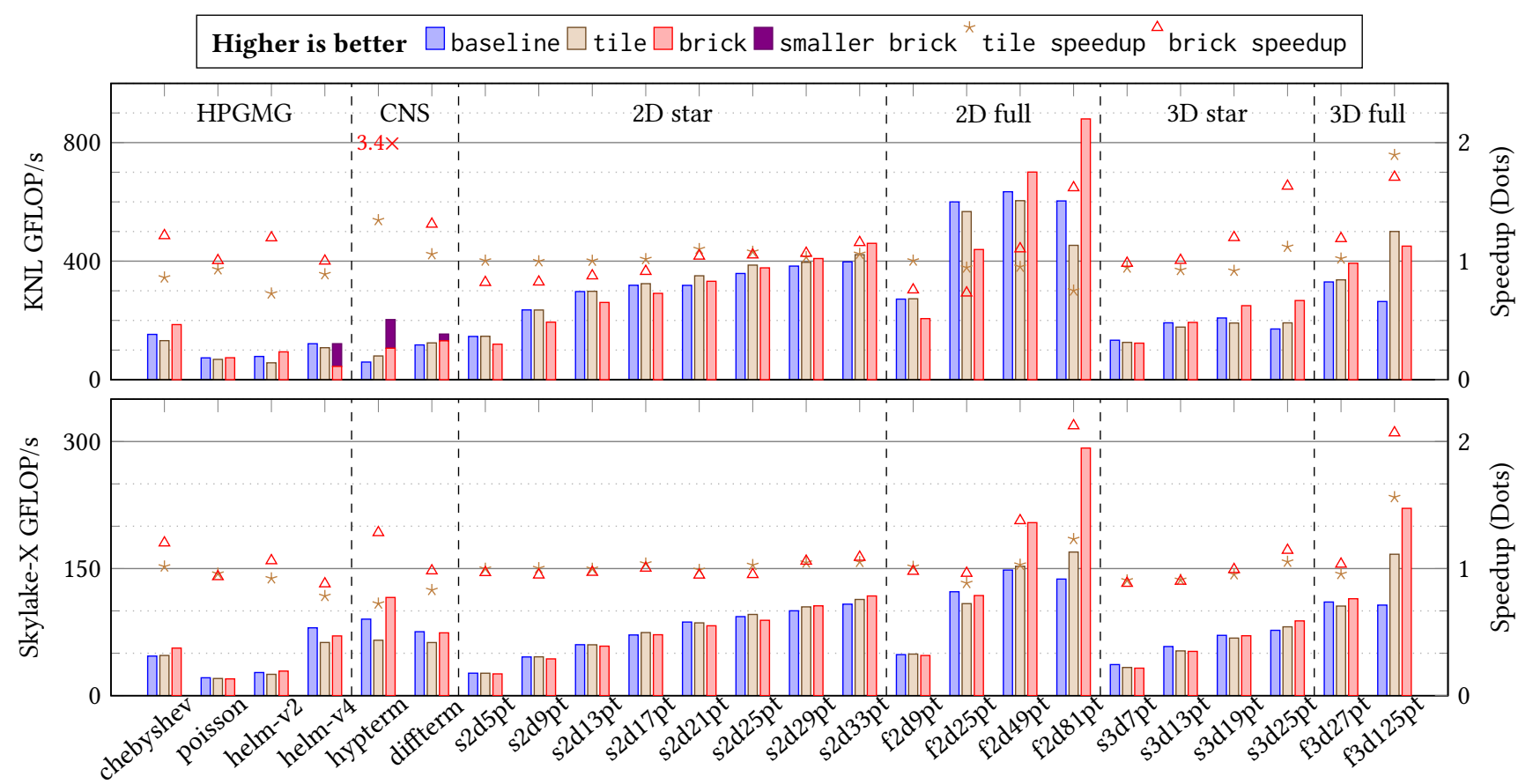

Figure 7: Performance on KNL and Skylake-X. For real-world stencil kernels, smaller blocking sizes may be beneficial because more input grids can put higher pressure on the cache. For higher-order and real-world stencils, the brick approach often provides the best speedup: On KNL, hypterm $-3.4 \times$ and f3d125pt $-1.9 \times$. On Skylake-X, hypterm $-1.3 \times$ and f3d125pt $-2.1 \times$.

streaming multiprocessor has a dedicated texture/L1 cache. The $\mathrm{P} 100$ has a theoretical peak single-precision performance of $9.3 \mathrm{TFLOP} / \mathrm{s}$, a peak double-precision performance of $4.7 \mathrm{TFLOP} / \mathrm{s}$, and a GPUSTREAM [9] bandwidth of $586 \mathrm{~GB} / \mathrm{s}$.

\subsection{Benchmarks and Proxy Codes}

We use three categories of stencil kernels of varying order shown in Table 1. The first category includes smoothers from the HPGMG benchmark suite [1]. The second category includes high-order stencil computations from the Compressible Navier-Stokes computation [12]. The third category includes synthetic stencils to capture the memory-compute ratio of different kinds of stencil shapes and radii. Many of the real stencils are a composition of these kernel patterns. Stencils are named according to their class ("f" or "s") and the number of points where each point is weighted individually. Class "s" refers to stencils for the Laplacian second derivatives, that only operate on elements along each of the axes (star-shaped) in each dimension. For Class "s", the stencil radius is just half the order; there are no off-axis points in the stencil. Class "f" refers to stencils for the "compact" Laplacian that touch all points in a cube (dense), with Manhattan distance equal to the radius. The radius is again just half the order; in some applications these stencils can produce more accurate solutions.

The baseline code is written in C. Our code generator generates code from the stencil written as python expressions in a python script, as in Figure 2(c). This specification can be used as a standalone DSL or as an intermediate output from the parser. The thresholds described in Section 3 are exposed as parameters to the

\begin{tabular}{|l|r|r||l|r|r|}
\hline Name & Grid & FLOPS & Name & Grid & FLOPS \\
\hline \multicolumn{7}{|c|}{ Stencils from HPGMG } \\
\hline chebyshev & 6 & 39 & poisson & 2 & 21 \\
\hline helm-v2 & 7 & 22 & helm-v4 & 7 & 115 \\
\hline \hline \multicolumn{6}{|c|}{ Stencils from CNS } \\
\hline hypterm & 13 & 358 & diffterm & 11 & 415 \\
\hline \hline \multicolumn{6}{|c|}{ 2D Star-Shaped Stencils } \\
\hline s2d5pt & 2 & 9 & s2d9pt & 2 & 17 \\
\hline s2d13pt & 2 & 25 & s2d17pt & 2 & 33 \\
\hline s2d21pt & 2 & 41 & s2d25pt & 2 & 49 \\
\hline s2d29pt & 2 & 49 & s2d33pt & 2 & 49 \\
\hline \hline \multicolumn{7}{|c|}{ 2D Full Stencils } \\
\hline f2d9pt & 2 & 17 & f2d25pt & 2 & 49 \\
\hline f2d49pt & 2 & 97 & f2d81pt & 2 & 161 \\
\hline \hline \multicolumn{7}{|c|}{ 3D Full Stencils } \\
\hline s3d7pt & 3D Star-Shaped Stencils \\
\hline s3d19pt & 2 & 13 & s3d13pt & 2 & 25 \\
\hline \hline \multicolumn{7}{|c|}{ 33 } & f3d125pt & 2 & 249 \\
\hline f3d27pt & 2 & 37 & s3d25pt & 2 & 49 \\
\hline
\end{tabular}

Table 1: Stencils used in experiments. Grid represents the number of grids the stencil operates on, while FLOPS represents the number of FLOPS performed per point.

code generator. We used $t_{1}=1.5$ (reuse estimate), $t_{2}=20$ (simultaneously active buffers), and $k=2$ (weight assigned to buffers) 


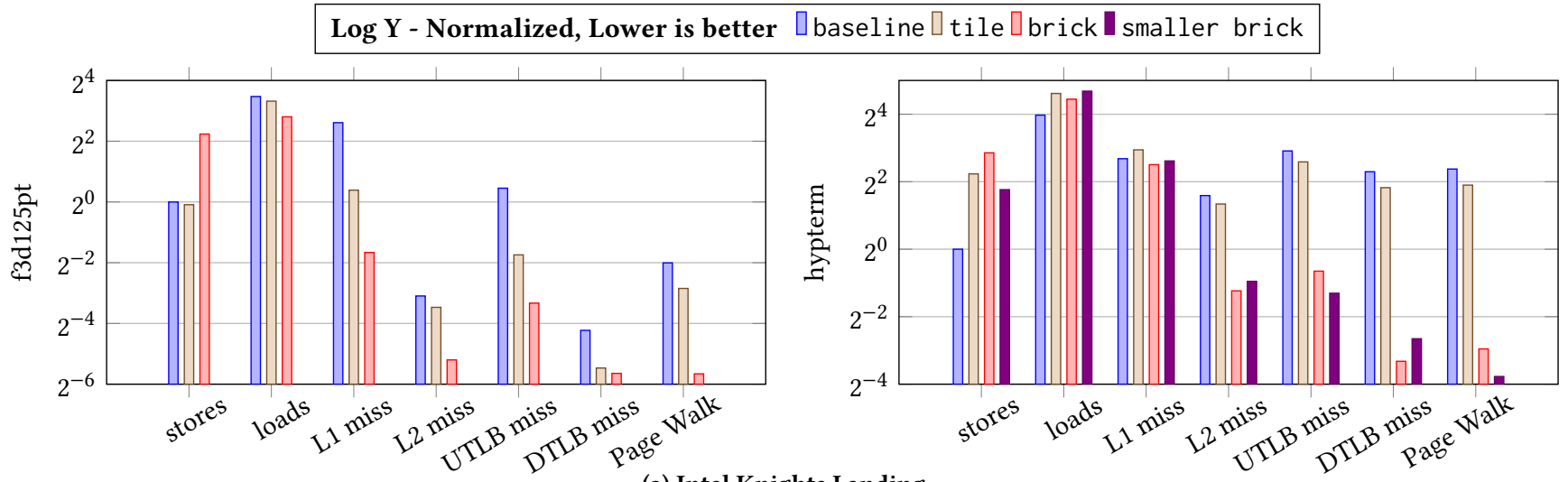

(a) Intel Knights Landing
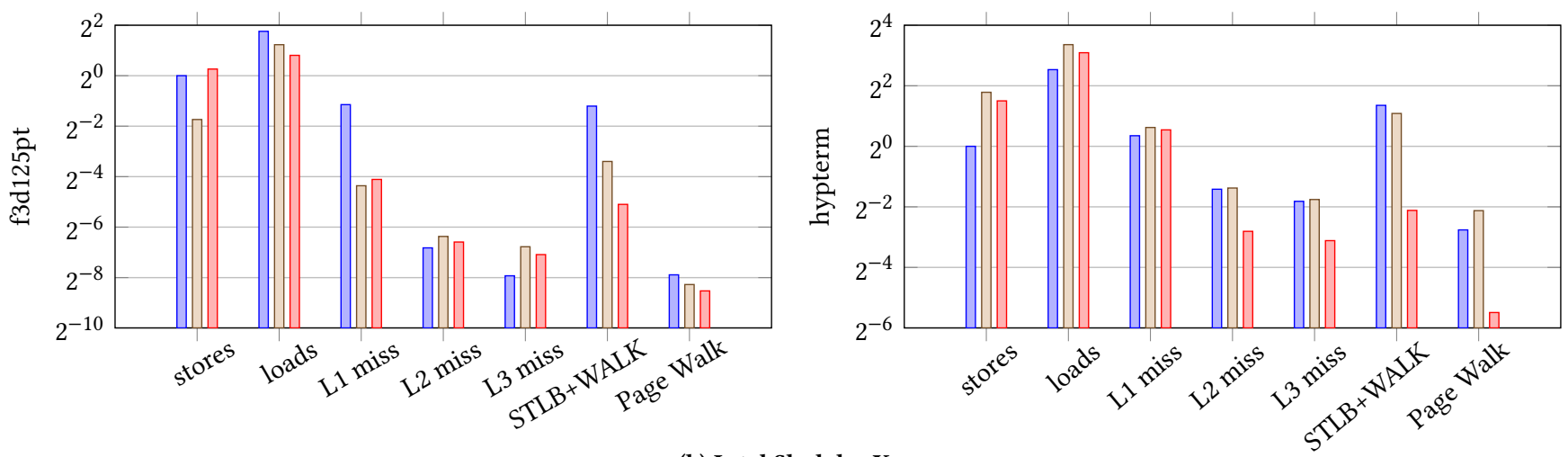

(b) Intel Skylake-X

Figure 8: Profiling metrics on KNL and Skylake-X. Each bar is the raw counter value, normalized to the baseline total number of stores. The generated code provides a dramatic reduction in cache and TLB misses and page walks. On KNL, L1 L2 and TLB misses are reduced as much as $19 \times, 7 \times$, and $49 \times$ respectively. On Skylake-X, we also reduced L1 misses by up to $8 \times$ and TLB-related metrics up to $14 \times$. These indicates much better data locality and cache reuse.

for our experiments. For all the stencils our code generator runs in under two seconds. Further discussion on the relationship between the brick library, the code generator, and our choices for these parameters is discussed in Section 5.5.

\subsection{CPU Performance: KNL and Skylake-X}

Figure 7 shows performance in GFLOP/s for the 24 stencils in our experiment, running on KNL and Skylake-X. We compare the performance of three code versions:

- a baseline version where the stencils are expressed as a gather. The stencils are tiled on all dimensions and parallelized using OpenMP. 2D stencils are parallelized using threads on the outermost tile control loop (J-dimension). 3D stencils are parallelized using threads on the two outermost tile control loops (K-, J-dimensions). The innermost tiled loop (I-dimension) is vectorized using \#pragma omp simd. For 3D stencils we use three different tile sizes: $4 \times 4 \times 4,4 \times 4 \times 8$, and $8 \times 8 \times 8$. For 2 D stencils we use two sizes: $8 \times 8$, and $4 \times 8$. This version reports the best performance out of these tile sizes.
- a tile version, that uses the same thread schedule and tiling as baseline but applies our code generator. Only reusing aligned loads is not applied as it attempts to be more general by keeping the array-format references. Vectorization is done using \#pragma omp simd.

- a brick version, where fine-grained data blocking is used [44] with our code generator. The thread schedule is kept the same, and vectorization also uses the OpenMP simd directive. Aligned data loads and merge are implemented using instrinsics to load vectors across brick boundaries. Reusing aligned load is applied. Here we try to separate the effect of different brick sizes by using brick to denote the performance for sizes $8 \times 8 \times 8$ and $8 \times 8$ and use smaller brick to represent other smaller sizes presents in the baseline.

The baseline and tile are compiled with either -02 or -0fast, whichever achieves the best performance. The brick version is compiled using -02 only.

The results for the two CPU architectures in Figure 7 suggest several observations. The trend of performance relative to the shape and size of the stencil can be shown in the synthetic stencils. For star-shaped stencils, both $2 \mathrm{D}$ and 3D, the relative performance of tile to the baseline increases with larger stencil diameters due 
to more temporal reuse. For lower order stencils, brick is slower than both other versions due to indexing overhead. However, since higher order stencils exhibit much higher temporal reuse, brick becomes the fastest version due to both the data blocking from bricks and operator reordering.

For the full stencils, on KNL, tile is able to obtain speedup on $3 \mathrm{D}$ but not on 2D stencils. This is due to 3D stencils exhibiting much higher reuse for each loaded input. Due to the alignment constraints, for each element read, f3d125pt is able to scatter to at most 25 points, while for $\mathrm{f} 2 \mathrm{~d} 81 \mathrm{pt}$ it can only have up to 9 points.

Although tile is often slower than brick, it is faster for f $3 \mathrm{~d} 125 \mathrm{pt}$ on KNL. Here, the generated code size becomes the bottleneck for brick because Equation 9 limits the region that can use loops. For tile, the code size for the entire kernel including outer loops is around $34 \mathrm{~KB}$ for $4 \times 4 \times 8$, which is when tile gives the best performance. Due to the extra indexing calculation and much wider peeled region from data indirection, the code size for bricks is $54 \mathrm{~KB}$. This is much higher than the L1 instruction cache size of 32KB.

We used Intel VTune Amplifier to obtain profiling results to further dissect the performance difference between different versions. We selected two of the complex stencils to show in Figure 8. When comparing brick to baseline on KNL, cache misses are reduced by up to $19 \times$, and TLB misses are reduced by up to $49 \times$ Similarly, on Skylake-X, brick also reduced L1 misses by up to $8 \times$ and TLB-related metrics up to $14 \times$. In addition, even though the code generator increases the number of stores, these appear to be serviced from L1 due to the decrease in cache misses as compared to baseline. The effect of better locality is more pronounced on KNL with more threads and much smaller cache per thread. For f3d125pt, our code generation can also reduce the total number of loads.

For the real-world stencils we noticed that tile offers similar or worse performance compared to baseline. This is due to the inherently higher L1 cache pressure for these stencils. Table 1 shows the number of grids referenced for each of these stencils. While we prefer each of the grids to be located in the fastest cache available for reuse, the buffers from vector scatter increase L1 pressure and detract from the better locality it provides. As seen in Figure 8, the L1 miss count is even across all versions of the code for hypterm. As noted previously, KNL threads have less cache capacity. baseline and tile tend to achieve the best performance on $4 \times 4 \times 8$ tiles or sometimes $4 \times 4 \times 4$ tiles. The smaller brick version, which relies on vector folding, improves KNL performance due to reduced stores and improved TLB behavior.

\subsection{GPU Performance: NVIDIA P100}

Figure 9 presents NVIDIA P100 performance. We compare the performance of three code variants:

- baseline version (a gather) that is tiled using the threadblock decomposition of CUDA, where each CUDA block is comprised of $4 \times 4 \times 32$ (K, J, I) threads for $3 \mathrm{D}$ or $8 \times 32$ for 2D. Each thread computes one stencil output. We also could spawn 32 threads for each block and iterate on the (K, J) dimension to further imitate the number of threads used for the brick version, but it is always slower.
- tile version that applies our code generation on a tiled thread-block, where it compute (K, J, I) elements using I threads. The tile size is the same as the baseline. Only aligned loads and reuse of those are not applied.

- brick version where fine-grained data blocking is used [44] with the code generator. The subdomain that each CUDA block will compute is the same as baseline, but only 32 threads (one warp) are in each block to compute all stencils in the subdomain, like a vector with width of 32 . We also tried smaller sizes such as $4 \times 4 \times 8$ and $4 \times 4 \times 4$ for real-world stencils; these are reported as smaller brick. Note that the I-dimension of these sizes are smaller than the vector width on the GPU which is the warpsize, 32.

In Figure 9, the baseline shows very little performance variation. This is because FLOP/s are limited by data dependences. Our approach reduces this bottleneck by exploiting input reuse. The NVCC compiler can generate code where the buffers introduced by vector scatter reside in registers. With sufficient registers, one element is read and scattered to multiple destinations in registers so that the write cost is low compared to read. The effect of holding buffers in registers is reflected in the drop of performance between (s2d21pt,s2d25pt) and (s3d13pt,s3d19pt). Also note that due to alignment, fewer registers are required than for gather. For example, for $\mathrm{f} 3 \mathrm{~d} 125 \mathrm{pt}$ only 25 destination register are used when scattering one input, whereas in gather 125 input are used.

We used NVProf to obtain several metrics related to memory performance for the $\mathrm{f} 3 \mathrm{~d} 125 \mathrm{pt}$ and hypterm stencils in Figure 10. Our code generator improves register and cache reuse significantly; the number of global loads are reduced by as much as $12 \times$. Higher cache reuse results in lower L1 pressure that contributes to reduction in the volume of global load traffic seen at L2. Some of the generated code temporarily stages values in local memory, which can be identified by the local load and store metric (L-Load/Store). The tile code also shows higher L2 traffic. The performance impact of these increases is significantly outweighed by the higher global and L2 loads of the baseline code. hypterm, one of the more complex stencils, exhibits increased register and cache pressure, resulting in extra local load and stores and HBM traffic even when using bricks. However, with a reduced brick size and vector folding, this effect is completely eliminated. It is possible that TLB behavior is improved on the GPU as it was on the CPU, but such metrics are unavailable in NVProf.

\subsection{Discussion}

In this subsection, we consider the performance impact of varying the configuration of the compiler and code generator to tease out contributions of different aspects of our approach. Suppose, for example, the brick library were used without the vector code generation. The brick library uses template expansion for address calculation and incorporates indirection to represent neighboring bricks; consequently, without code generation, backend compilers will introduce redundant index calculations and cannot vectorize on CPUs. In fact, stencils in the naive brick library version might even be slower than the baseline. For the more compute-intensive stencils such as the $125 \mathrm{pt}$, our code generator provides an $18 \times$ speedup when compared to the naive brick library version on KNL. 


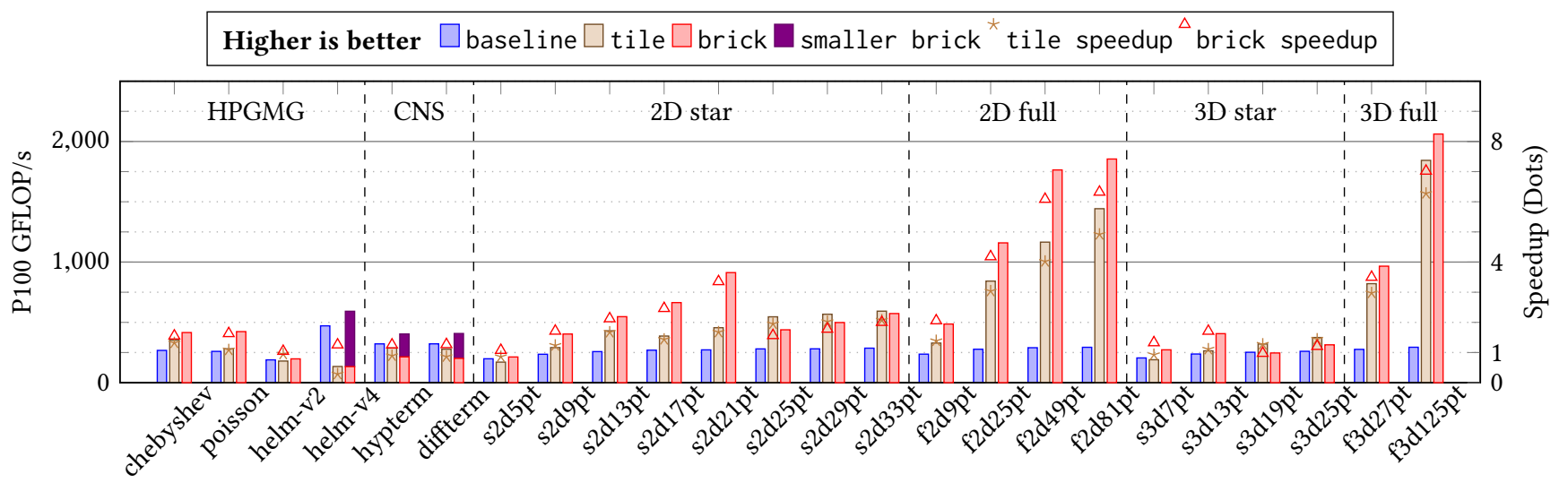

Figure 9: Performance on NVIDIA P100. Brick code generation produces the best performance for many of the stencils. For synthetic stencils, using either 32 threads or full block results has little effect, but smaller block sizes reduce cache pressure for real stencils. Bricks speedup many stencils, for example poisson (1.6×) and f3d125pt (7.0×).

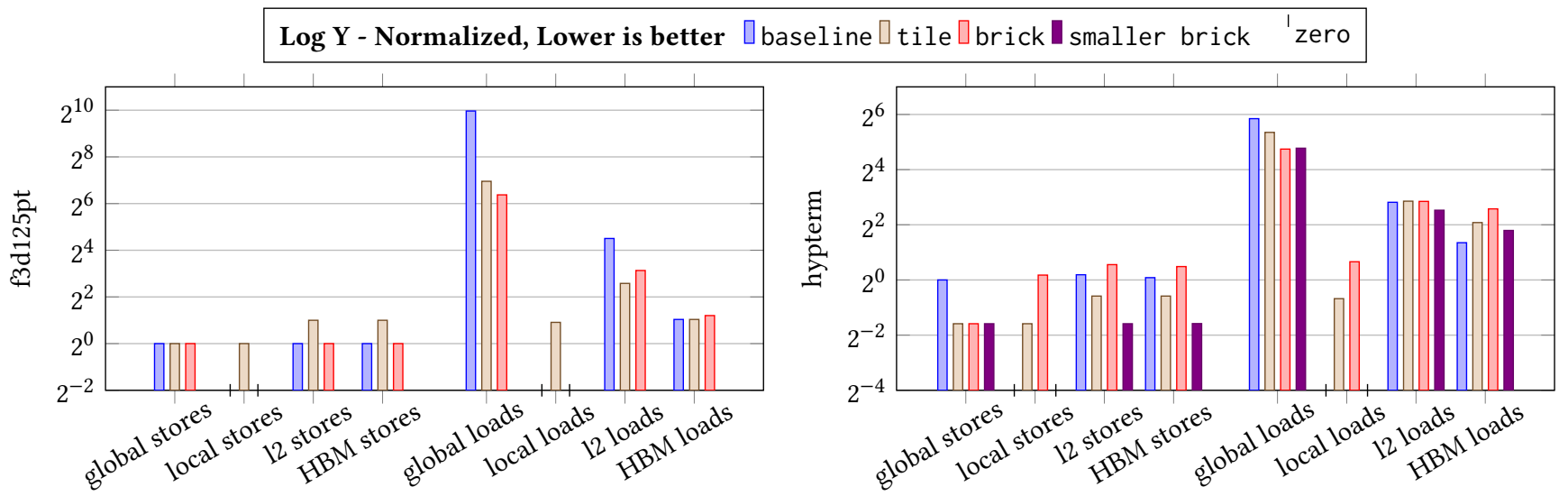

Figure 10: Profiling metrics on P100. Each bar represent transaction count normalized to the total number of global stores of baseline. brick version reduces global loads by up to $12 \times$ and L2 loads by up to $2.6 \times$ implying much better locality.

Even on P100, where these issues are less pronounced, the fully optimized code is $7.5 \times$ faster.

The thresholds used in the experiment are fixed using lenient values. These thresholds are especially relaxed for simple stencils. Our reuse estimate of $t_{1}=1.5$ applies to all associative operators that have reuse potential in our tests. Aside from the value 1 that denotes no potential reuse, the lowest reuse potential is $12 / 7 \approx 1.86$ in helm-v2. This is due to the fact that helm-v2 contains terms using multiple grid references that are not perfectly reused after applying the shifts.

Our estimate of $t_{2}=20, k=2$ is a proxy for how much register pressure the algorithm incurs. These criteria are also only effective for complex stencils and are rarely met for simple stencils. All synthetic stencils have a value for Equation 5 of 3. For complex stencils like helm-v4 and hypterm, it is theoretically possible to reach this limit. However, this does not happen as our code generator strategy requires reuse between operators in one stage but limits the number of operators that can be put in one stage. This requirement may be proven to be too greedy and further tuning of these parameters is yet to be explored.

\section{RELATED WORK}

Optimization efforts for stencil computations can be broadly classified as memory access optimization techniques, and optimization methods to improve computation, although in practice, there is often significant interaction between them.

Most of the optimization effort has focused on stencils applied on large grids that are usually bound by capacity or compulsory cache misses, leading to a variety of studies on spatial and temporal tiling [6, 7, 11, 13, 19-23, 26-28, 33-36, 38, 41, 45]. In addition, domain-specific compilers have recently been developed for parallel code generation from a stylized stencil specification [4, 30, 42, 43] or from a code excerpt [16].

The aforementioned tiling techniques have focused on loop or iteration space tiling. In addition to loop tiling, researchers have also tiled or blocked data (space). Data along with loop tiling efforts have been addressed by $[2,17,31,40]$. TiDA [31] uses coarse-grained data blocking, where the entire grid is tiled into sub-grids, each with its own ghost zone. Fine-grained data blocking is explored in Bricks [44] and Briquettes [17], YASK [40] and RTM on the Cell processor [2]. All the fine-grained blocking techniques targeted 
large, compute-intensive stencils, and the small data blocks (bricks) do not have per-block ghost zones.

The fine-grained data blocks used in our research are similar to briquettes in [17], but there is significant difference in our approaches. Briquettes were designed to perform 3D stencils split into $1 \mathrm{D}$ stencils, thus requiring multiple sweeps to compute the output. Furthermore, a data transpose was required between each 1D stencil sweep to ensure good SIMDization. Their code generation required data staging tailored for $1 \mathrm{D}$ stencils. In contrast to Briquettes, we optimize 3D stencils without manual dimensional splitting and perform complex stencil reordering in addition to fine-grained data blocking to improve computation by reducing reads and improving SIMDization.

YASK is a C++ template-based approach to generating code for large stencils with fine-grained data blocks. YASK autotuned their data block size, and used smaller data blocks than our method (e.g. $2 \times 2 \times 4$ instead of $8^{3}$ ). They can generate code for clusters of vectors using unrolling and common expression elimination to improve reuse, which is less feasible for complex stencils. They did not directly target stencil reordering as presented in our paper.

Stencil reordering, one of the main characteristics of our approach, has been explored in different ways. Manual optimization of stencil computations has led to techniques such as semi-stencils to reduce loads [8] and using common subexpression elimination after unrolling to reduce floating-point computations, improve register reuse, reduce register pressure, or improve instruction level parallelism [5, 7, 25]. Some works target specific properties of the associative operation in stencils. Deitz et al. [10] describes an automated approach to common subexpression elimination for sum-ofproduct computation and is not applicable to uniquely weighted or variable coefficient stencils where no common subexpressions exist. Basu et al. [3] uses partial sums to reorder constant-coefficient isotropic stencils. Stock et al. [29] uses statement splitting to enable loop shifting to expose reuse of the same input and autotuning to determine the shift amount. They also do not target code generation for the GPU. In comparison, the research presented in this paper illustrates a new powerful stencil reordering method which works on general stencils without manual optimizations. Our method targets tiled stencil computation to improve cache and register reuse, and is designed to work with fine-grained data blocking on modern architectures with wide SIMD units.

High-order PDEs can also be implemented as dense matrix operations as in [32]; this paper and other previous compiler/codegeneration research treats higher-order stencils as computations on structured grids. Using dense linear algebra primitives, although technically feasible for finite difference and finite volume methods, would be inefficient for our stencils as most of the entries in the resultant matrices would be zero.

\section{CONCLUSION}

High-order stencil computations are simultaneously best-suited to the trends in computer architecture (limited bandwidth coupled with high arithmetic intensity) and most-often underperforming. To that end, in this paper, we introduce a novel compiler optimization to exploit reuse and vectorization in block stencil computations. When coupled with a fine-grained blocked data layout (bricks) this produces code that reduces vector loads and alignment operations, exposes opportunities to eliminate redundant computation, and reduces the data footprint of stencils in the memory hierarchy. We show our approach improves the performance of real stencils compared to a tiled baseline by up to $3.4 \times$ on a Intel Knights Landing (Xeon Phi) processor, up to $1.3 \times$ on Intel Xeon Skylake-X, and up to $1.6 \times$ on NVIDIA P100.

\section{ACKNOWLEDGMENTS}

This research was supported by the Exascale Computing Project (17-SC-20-SC), a joint project of the U.S. Department of Energy's Office of Science and National Nuclear Security Administration. This research used resources in Lawrence Berkeley National Laboratory and the National Energy Research Scientific Computing Center, which are supported by the U.S. Department of Energy Office of Sciences Advanced Scientific Computing Research program under contract number DE-AC02-05CH11231.

\section{REFERENCES}

[1] 2016. High-Performance Geometric Multigrid. http://hpgmg.org

[2] Mauricio Araya-Polo, Félix Rubio, Raúl de la Cruz, Mauricio Hanzich, José María Cela, and Daniele Paolo Scarpazza. 2009. 3D Seismic Imaging Through Reversetime Migration on Homogeneous and Heterogeneous Multi-core Processors. Sci. Program. 17, 1-2 (Jan. 2009), 185-198. https://doi.org/10.1155/2009/382638

[3] Protonu Basu, Mary Hall, Samuel Williams, Brian Van Straalen, Leonid Oliker, and Phillip Colella. 2015. Compiler-directed transformation for higher-order stencils. In Parallel and Distributed Processing Symposium (IPDPS), 2015 IEEE International. IEEE, 313-323.

[4] M. Christen, O. Schenk, and H. Burkhart. 2011. PATUS: A Code Generation and Autotuning Framework for Parallel Iterative Stencil Computations on Modern Microarchitectures. In Parallel Distributed Processing Symposium (IPDPS). https: //doi.org/10.1109/IPDPS.2011.70

[5] Kaushik Datta. 2009. Auto-tuning Stencil Codes for Cache-Based Multicore Platforms. Ph.D. Dissertation. EECS Department, University of California, Berkeley.

[6] Kaushik Datta, Shoaib Kamil, Samuel Williams, Leonid Oliker, John Shalf, and Katherine Yelick. 2009. Optimization and Performance Modeling of Stencil Computations on Modern Microprocessors. SIAM Rev. 51, 1 (2009), 129-159.

[7] Kaushik Datta, Mark Murphy, Vasily Volkov, Samuel Williams, Jonathan Carter, Leonid Oliker, David Patterson, John Shalf, and Katherine Yelick. 2008. Stencil Computation Optimization and Auto-Tuning on State-of-the-art Multicore Architectures. In Supercomputing (SC).

[8] Raúl De La Cruz, Mauricio Araya-Polo, and José María Cela. 2010. Introducing the Semi-stencil Algorithm. In International Conference on Parallel Processing and Applied Mathematics: Part I (PPAM). 11.

[9] Tom Deakin, James Price, Matt Martineau, and Simon McIntosh-Smith. 2016. GPU-STREAM v2. 0: benchmarking the achievable memory bandwidth of manycore processors across diverse parallel programming models. In International Conference on High Performance Computing. Springer, 489-507.

[10] Steven J Deitz, Bradford L Chamberlain, and Lawrence Snyder. 2001. Eliminating redundancies in sum-of-product array computations. In Proceedings of the 15th international conference on Supercomputing. ACM, 65-77.

[11] Craig C. Douglas, Jonathan Hu, Markus Kowarschik, Ulrich Rüde, and Christian Weiss. 2000. Cache Optimization for Structured and Unstructured Grid Multigrid. Elect. Trans. Numer. Anal 10 (2000), 21-40.

[12] Matthew Emmett, Weiqun Zhang, and John B Bell. 2014. High-order algorithms for compressible reacting flow with complex chemistry. Combustion Theory and Modelling 18, 3 (2014), 361-387.

[13] M. Frigo and V. Strumpen. 2005. Evaluation of cache-based superscalar and cacheless vector architectures for scientific computations. In Proc. ACM International Conference on Supercomputing (ICS).

[14] P. Ghysels, P. Kosiewicz, and W. Vanroose. 2012. Improving the arithmetic intensity of multigrid with the help of polynomial smoothers. Numerical Linear Algebra with Applications 19, 2 (2012), 253-267.

[15] Tom Henretty, Kevin Stock, Louis-Noël Pouchet, Franz Franchetti, J Ramanujam, and P Sadayappan. 2011. Data layout transformation for stencil computations on short-vector simd architectures. In Compiler Construction. Springer, 225-245.

[16] Justin Holewinski, Louis-Noël Pouchet, and P. Sadayappan. 2012. Highperformance code generation for stencil computations on GPU architectures. In International Conference on Supercomputing (ICS). 
[17] Jagan Jayaraj. 2013. A strategy for high performance in computational fluid dynamics. Ph.D. Dissertation. University of Minnesota.

[18] Jagan Jayaraj, Pei-Hung Lin, Paul R Woodward, and Pen-Chung Yew. 2014. CFD builder: A library builder for computational fluid dynamics. In Parallel \& Distributed Processing Symposium Workshops (IPDPSW), 2014 IEEE International. IEEE, $1029-1038$.

[19] Markus Kowarschik and Christian WeiSS. 2001. DiMEPACK - A Cache-Optimized Multigrid Library. In International Conference on Parallel and Distributed Processing Techniques and Applications (PDPTA), volume I.

[20] Sriram Krishnamoorthy, Muthu Baskaran, Uday Bondhugula, J. Ramanujam Atanas Rountev, and P Sadayappan. 2007. Effective automatic parallelization of stencil computations. In Proc. ACM SIGPLAN conference on Programming language design and implementation (PLDI).

[21] J. McCalpin and D. Wonnacott. 1999. Time skewing: A value-based approach to optimizing for memory locality. Technical Report DCS-TR-379. Department of Computer Science, Rutgers University.

[22] Paulius Micikevicius. 2009. 3D Finite Difference Computation on GPUs Using CUDA. In Proceedings of 2nd Workshop on General Purpose Processing on Graphics Processing Units (GPGPU-2). 6.

[23] Anthony Nguyen, Nadathur Satish, Jatin Chhugani, Changkyu Kim, and Pradeep Dubey. 2010. 3.5-D Blocking Optimization for Stencil Computations on Modern CPUs and GPUs. In Proc. ACM/IEEE International Conference for High Performance Computing, Networking, Storage and Analysis (SC).

[24] Prashant Singh Rawat, Fabrice Rastello, Aravind Sukumaran-Rajam, Louis-Noël Pouchet, Atanas Rountev, and P. Sadayappan. 2018. Register Optimizations for Stencils on GPUs. In Proceedings of the 23rd ACM SIGPLAN Symposium on Principles and Practice of Parallel Programming (PPoPP '18). ACM, New York, NY, USA, 168-182. https://doi.org/10.1145/3178487.3178500

[25] Prashant Singh Rawat, Aravind Sukumaran-Rajam, Atanas Rountev, Fabrice Rastello, Louis-Noël Pouchet, and P. Sadayappan. 2018. Associative Instruction Reordering to Alleviate Register Pressure. In Proceedings of the International Conference for High Performance Computing, Networking, Storage, and Analysis (SC '18). IEEE Press, Piscataway, NJ, USA, Article 46, 13 pages. http://dl.acm.org/ citation.cfm?id=3291656.3291718

[26] G. Rivera and C. Tseng. 2000. Tiling Optimizations for 3D Scientific Computations. In Supercomputing (SC)

[27] S. Sellappa and S. Chatterjee. 2004. Cache-Efficient Multigrid Algorithms. International fournal of High Performance Computing Applications 18, 1 (2004), $115-133$.

[28] Y. Song and Z. Li. 1999. New tiling techniques to improve cache temporal locality. In Proc. ACM SIGPLAN Conference on Programming Language Design and Implementation (PLDI).

[29] Kevin Stock, Martin Kong, Tobias Grosser, Louis-Noël Pouchet, Fabrice Rastello, Jagannathan Ramanujam, and Ponnuswamy Sadayappan. 2014. A framework for enhancing data reuse via associative reordering. In ACM SIGPLAN Notices, Vol. 49. ACM, 65-76.

[30] Yuan Tang, Rezaul Alam Chowdhury, Bradley C. Kuszmaul, Chi-Keung Luk, and Charles E. Leiserson. 2011. The pochoir stencil compiler. In ACM symposium on Parallelism in algorithms and architectures.

[31] Didem Unat, Tan Nguyen, Weiqun Zhang, Muhammed Nufail Farooqi, Burak Bastem, George Michelogiannakis, Ann Almgren, and John Shalf. 2016. TiDA High-Level Programming Abstractions for Data Locality Management. Springer International Publishing, Cham, 116-135.

[32] Jerry E. Watkins, Joshua Romero, and Antony Jameson. 2016. Multi-GPU, Implicit Time Stepping for High-order Methods on Unstructured Grids. In 46th AIAA Fluid Dynamics Conference. American Institute of Aeronautics and Astronautics. https://doi.org/10.2514/6.2016-3965

[33] Gerhard Wellein, Georg Hager, Thomas Zeiser, Markus Wittmann, and Holger Fehske. 2009. Efficient Temporal Blocking for Stencil Computations by Multicore-Aware Wavefront Parallelization. In International Computer Software and Applications Conference. https://doi.org/10.1109/COMPSAC.2009.82

[34] S. Williams, J. Carter, L. Oliker, J. Shalf, and K. Yelick. 2008. Lattice Boltzmann Simulation Optimization on Leading Multicore Platforms. In Interational Conference on Parallel and Distributed Computing Systems (IPDPS).

[35] Samuel Williams, Leonid Oliker, Jonathan Carter, and John Shalf. 2011. Extracting ultra-scale Lattice Boltzmann performance via hierarchical and distributed autotuning. In Supercomputing (SC).

[36] S. Williams, J. Shalf, L. Oliker, S. Kamil, P. Husbands, and K. Yelick. 2006. The potential of the Cell processor for scientific computing. In Proc. Conference on Computing Frontiers.

[37] S. Williams, A. Watterman, and D. Patterson. 2009. Roofline: An Insightful Visual Performance Model for Floating-Point Programs and Multicore Architectures. Commun. ACM (April 2009).

[38] D. Wonnacott. 2000. Using Time Skewing to Eliminate Idle Time due to Memory Bandwidth and Network Limitations. In Proc. Interational Conference on Parallel and Distributed Computing Systems.
[39] C. Yount. 2015. Vector Folding: Improving Stencil Performance via Multidimensional SIMD-vector Representation. In 2015 IEEE 17th International Conference on High Performance Computing and Communications, 2015 IEEE 7th International Symposium on Cyberspace Safety and Security, and 2015 IEEE 12th International Conference on Embedded Software and Systems. 865-870.

[40] Charles Yount, Josh Tobin, Alexander Breuer, and Alejandro Duran. 2016. YASKyet Another Stencil Kernel: A Framework for HPC Stencil Code-generation and Tuning. In Proceedings of the Sixth International Workshop on Domain-Specific Languages and High-Level Frameworks for HPC (WOLFHPC '16). 10.

[41] T. Zeiser, G. Wellein, A. Nitsure, K. Iglberger, U. Rude, and G. Hager. 2008. Introducing a parallel cache oblivious blocking approach for the lattice Boltzmann method. Progress in Computational Fluid Dynamics 8 (2008).

[42] N. Zhang, M. Driscoll, C. Markley, S. Williams, P. Basu, and A. Fox. 2017. Snowflake: A Lightweight Portable Stencil DSL. In 2017 IEEE International Parallel and Distributed Processing Symposium Workshops (IPDPSW). 795-804.

[43] Yongpeng Zhang and Frank Mueller. 2012. Auto-generation and auto-tuning of 3D stencil codes on GPU clusters. In International Symposium on Code Generation and Optimization (CGO).

[44] T. Zhao, S. Williams, M. Hall, and H. Johansen. 2018. Delivering PerformancePortable Stencil Computations on CPUs and GPUs Using Bricks. In 2018 IEEE/ACM International Workshop on Performance, Portability and Productivity in HPC (P3HPC). 59-70. https://doi.org/10.1109/P3HPC.2018.00009

[45] Xing Zhou, Jean-Pierre Giacalone, María Jesús Garzarán, Robert H. Kuhn, Yang $\mathrm{Ni}$, and David Padua. 2012. Hierarchical overlapped tiling. In Proc. International Symposium on Code Generation and Optimization (CGO).

[46] Gerhard Zumbusch. 2013. Vectorized Higher Order Finite Difference Kernels. In Proceedings of the 11th International Conference on Applied Parallel and Scientific Computing (PARA'12). Springer-Verlag, Berlin, Heidelberg, 343-357. https://doi. org/10.1007/978-3-642-36803-5_25 


\section{Appendix: Artifact Description/Artifact Evaluation}

\begin{abstract}
SUMMARY OF THE EXPERIMENTS REPORTED
We developed a code generator using our approach described in the paper and applied to the 24 stencil kernels shown in the experiment section. We run the code generator and compile the output and baseline code individually for each of the platforms we use. These code variants are compiled using Intel Compiler 2019 and run on the KNL node on NERSC's Cori. They are also compiled using Intel Compiler 2019 and run on Notchpeak Skylake-X node from University of Utah's CHPC. For GPU, they are compiled using CUDA 9.2 and run on Kingspeak P100 node from University of Utah's CHPC.
\end{abstract}

\section{ARTIFACT AVAILABILITY}

Software Artifact Availability: All author-created software artifacts are maintained in a public repository under an OSI-approved license.

Hardware Artifact Availability: There are no author-created hardware artifacts.

Data Artifact Availability: There are no author-created data artifacts.

Proprietary Artifacts: None of the associated artifacts, authorcreated or otherwise, are proprietary.

List of URLs and/or DOIs where artifacts are available:

https://bitbucket.org/ztuowen/vecscatter-artifact/sr , $\hookrightarrow$ c/master/

\section{BASELINE EXPERIMENTAL SETUP, AND MODIFICATIONS MADE FOR THE PAPER} P100

Relevant hardware details: KNL 7250, Xeon Gold 6130, Nvidia

Compilers and versions: Intel C++ Compiler 2019.3 (GCC 8.2 compatibility) (KNL), Intel C++ Compiler 2019.2 (GCC 8.1 compatibility) (Skylake-X), CUDA 9.2 (GCC 6.4.0)

Libraries and versions: Intel OpenMP corresponding to each compiler version

Paper Modifications: No hardware or software modification. Our code generation generates source code that is input to the available compiler. tion.

Output from scripts that gathers execution environment informa-

P100 node:

LMOD_FAMILY_COMPILER_VERSION $=6.4 .0$

SLURM_NODELIST $=k p 362$

SLURM_CHECKPOINT_IMAGE_DIR=/var/slurm/checkpoint
MANPATH=/uufs/chpc. utah. edu/sys/installdir/gcc/6.4.0 $\hookrightarrow$ /share/man:/uufs/chpc.utah.edu/sys/installdir/lm」 $\hookrightarrow$ od/7.7.29/share/man:/usr/kerberos/man:/usr/local

$\hookrightarrow$ /share/man:/usr/share/man/overrides:/usr/share/m」

$\hookrightarrow$ an:/uufs/kingspeak. peaks/sys/pkg/slurm/std/share 」

$\hookrightarrow \quad /$ man

SLURM_JOB_NAME=bash

XDG_SESSION_ID $=87380$

_ModuleTable003_=UEMtMTgvQ29tcGlsZXIva3AvZ2NjLzYuNC4

$\hookrightarrow \quad$ wIiwiL3V1ZnMvY2hwYy51dGFoLmVkdS9zeXMvbW9kdWxlZml

$\hookrightarrow$ sZXMvQ0hQQy0xOC9Db21waWxlci9nY2MvNi40LjAiLCIvdXV」

$\hookrightarrow \quad$ mcy9jaHBjLnV0YWguZWR1L3N5cy9tb2R1bGVmaWxlcy9DSFB

$\hookrightarrow \quad$ DLTE4L0NvbXBpbGVyL2N1ZGEvOS4yI iwiL3V1ZnMvY2hwYy5」

$\hookrightarrow \quad$ 1dGFoLmVkdS9zeXMvbW9kdWxlZmlsZXMvQ0hQQy0xOC9MaW5 」

$\hookrightarrow$ 1eCIsIi91dWZzL2NocGMudXRhaC51ZHUvc3lzL21vZHVsZWZ 」

$\hookrightarrow$ pbGVZLONIUEMtMTgVQ29yZSIsI i91dWZzL2NocGMudXRhaC5

$\hookrightarrow$ 1ZHUvc3lzL2luc3RhbGxkaXIvbG1vZC83LjcuMjkvbW9kdWx 」

$\hookrightarrow \quad 1 Z m l s Z X M v Q 29 y Z S I s f S x b I n N 5 c 3 R 1 b U J h c 2 V N U E F U S C J d P S I$

$\hookrightarrow \quad$ vdXVmcy9jaHBjLnV0YWguZWR1L3N5cy9tb2R1bGVmaWxl

HOSTNAME $=\mathrm{kp} 362$

SLURM_TOPOLOGY_ADDR=kp362

SLURMD_NODENAME $=\mathrm{kp} 362$

LMOD_FAMILY_CUDA $=$ cuda

SLURM_PRIO_PROCESS $=0$

CUDA_BINDIR=/uufs/chpc.utah.edu/sys/installdir/cuda/ 」 $\hookrightarrow \quad 9.2 .148 / \mathrm{bin}$

SLURM_SRUN_COMM_PORT $=45416$

CUDA_INCDIR=/uufs/chpc.utah.edu/sys/installdir/cuda/ 」

$\hookrightarrow \quad$ 9.2.148/include

__LMOD_REF_COUNT_MODULEPATH=/uufs/chpc.utah.edu/sys / 」

$\hookrightarrow$ modulefiles/CHPC-18/Compiler/kp/gcc/6.4.0:1;/uuf 」

$\hookrightarrow$ s/chpc.utah.edu/sys/modulefiles/CHPC-18/Compiler 」

$\hookrightarrow /$ gcc/6.4.0:1;/uufs/chpc.utah.edu/sys/modulefiles」

$\hookrightarrow /$ /CHPC-18/Compiler/cuda/9.2:1;/uufs/chpc.utah.edu

$\hookrightarrow$ /sys/modulefiles/CHPC-18/Linux:1;/uufs/chpc.utah」

$\hookrightarrow$.edu/sys/modulefiles/CHPC-18/Core: 1 ; /uufs/chpc.u

$\hookrightarrow$ tah.edu/sys/installdir/lmod/7.7.29/modulefiles/C J

$\hookrightarrow$ ore: 1

TERM=rxvt-unicode-256color

SHELL=/bin/bash

CUDA_LIBDIR=/uufs/chpc. utah.edu/sys/installdir/cuda/ 」

$\hookrightarrow \quad 9.2 .148 / 1$ ib64

HISTSIZE $=1000$

SLURM_PTY_WIN_ROW=55

SLURM_JOB_QOS=QOS

LMOD_SYSTEM_DEFAULT_MODULES $=c h p c$

MODULEPATH_ROOT=/uufs/chpc.utah.edu/sys/modulefiles/ 」

$\hookrightarrow \quad$ CHPC -18

SSH_CLIENT=155.98.69.105 4543222

SLURM_TOPOLOGY_ADDR_PATTERN=node

TMPDIR $=/ \mathrm{tmp}$ 
CUDA_ROOTDIR=/uufs/chpc.utah.edu/sys/installdir/cuda 」 $\leftrightarrow \quad / 9.2 .148$

LMOD_FAMILY_CUDA_VERSION=9.2

LMOD_PKG=/uufs/chpc.utah.edu/sys/installdir/lmod/7.7 ।

$\hookrightarrow \quad .29$

SLURM_CPU_BIND_VERBOSE $=$ quiet

QTDIR=/usr/lib64/qt-3.3

QTINC=/usr/lib64/qt-3.3/include

LMOD_VERSION=7.7.29

SSH_TTY=/dev/pts $/ 82$

__LMOD_REF_COUNT_LOADEDMODULES=chpc/1.0:1; cuda/9.2:1」

$\hookrightarrow \quad ; \mathrm{gcc} / 6.4 .0: 1$

SLURM_CPU_BIND_LIST =

QT_GRAPHICSSYSTEM_CHECKED $=1$

OSVER=7.6.1810

USER=USER

SLURM_NNODES $=1$

LD_LIBRARY_PATH=/uufs/chpc.utah.edu/sys/installdir/g 」

$\hookrightarrow \mathrm{cc} / 6.4 .0 / \mathrm{ib64:/uufs/chpc.utah.edu/sys/installdi}$ 」

$\hookrightarrow$ r/gcc/6.4.0/lib:/uufs/chpc.utah.edu/sys/installd

$\hookrightarrow \mathrm{ir} /$ cuda/9.2.148/lib64:/uufs/kingspeak.peaks/sys/」

$\hookrightarrow \mathrm{pkg} / \mathrm{slurm} / \mathrm{std} / \mathrm{lib}$
LS_COLORS $=r s=0: \mathrm{di}=38 ; 5 ; 27: \ln =38 ; 5 ; 51: \mathrm{mh}=44 ; 38 ; 5 ; 15: \mathrm{p}$ 」

$\hookrightarrow \quad \mathrm{i}=40 ; 38 ; 5 ; 11: \mathrm{so}=38 ; 5 ; 13: \mathrm{do}=38 ; 5 ; 5: \mathrm{bd}=48 ; 5 ; 232 ; 38$ 」

$\hookrightarrow \quad ; 5 ; 11: \mathrm{cd}=48 ; 5 ; 232 ; 38 ; 5 ; 3:$ or $=48 ; 5 ; 232 ; 38 ; 5 ; 9: \mathrm{mi}=0$ 」

$\hookrightarrow \quad 5 ; 48 ; 5 ; 232 ; 38 ; 5 ; 15:$ su $=48 ; 5 ; 196 ; 38 ; 5 ; 15: \mathrm{sg}=48 ; 5 ; 1$ 」

$\hookrightarrow \quad 1 ; 38 ; 5 ; 16: \mathrm{ca}=48 ; 5 ; 196 ; 38 ; 5 ; 226: \mathrm{tw}=48 ; 5 ; 10 ; 38 ; 5 ; 1$ 」

$\hookrightarrow 6: \mathrm{ow}=48 ; 5 ; 10 ; 38 ; 5 ; 21: \mathrm{st}=48 ; 5 ; 21 ; 38 ; 5 ; 15: \mathrm{ex}=38 ; 5 ;$ 」

$\hookrightarrow \quad 34: * . \operatorname{tar}=38 ; 5 ; 9: * . \operatorname{tgz}=38 ; 5 ; 9: * . \operatorname{arc}=38 ; 5 ; 9: * . \operatorname{arj}=$ 」

$\hookrightarrow 38 ; 5 ; 9: *$. taz $=38 ; 5 ; 9: * .1$ ha $=38 ; 5 ; 9: * .1 z 4=38 ; 5 ; 9: *$.」

$\hookrightarrow \quad l z h=38 ; 5 ; 9: * .1 z m a=38 ; 5 ; 9: * . t l z=38 ; 5 ; 9: * . t x z=38 ; 5$ 」

$\hookrightarrow \quad ; 9: * . t z o=38 ; 5 ; 9: * . t 7 z=38 ; 5 ; 9: * . z i p=38 ; 5 ; 9: * . z=38$ ।

$\hookrightarrow ; 5 ; 9: * . z=38 ; 5 ; 9: * . d z=38 ; 5 ; 9: * . g z=38 ; 5 ; 9: * .1 r z=38$

$\hookrightarrow \quad ; 5 ; 9: * .1 z=38 ; 5 ; 9: * .1 z 0=38 ; 5 ; 9: * . x z=38 ; 5 ; 9: * . b z 2=$

$\hookrightarrow \quad 38 ; 5 ; 9: * . b z=38 ; 5 ; 9: *$. tbz $=38 ; 5 ; 9: * . t b z 2=38 ; 5 ; 9: *$.

$\hookrightarrow \mathrm{tz}=38 ; 5 ; 9: * . \mathrm{deb}=38 ; 5 ; 9: * . \mathrm{rpm}=38 ; 5 ; 9: *$.jar $=38 ; 5 ; 9$,

$\hookrightarrow \quad: *$.war $=38 ; 5 ; 9: *$. ear $=38 ; 5 ; 9: * . \operatorname{sar}=38 ; 5 ; 9: *$. $r a r=38$ 」

$\hookrightarrow \quad ; 5 ; 9: *$.alz=38;5;9:*.ace $=38 ; 5 ; 9: * . z o o=38 ; 5 ; 9: * . c p$ ।

$\hookrightarrow \quad$ io $=38 ; 5 ; 9: * .7 z=38 ; 5 ; 9: * . r z=38 ; 5 ; 9: * . c a b=38 ; 5 ; 9: *$ 」

$\hookrightarrow \quad . j p g=38 ; 5 ; 13: * . j p e g=38 ; 5 ; 13: *$.gi $f=38 ; 5 ; 13: * . b m p=$

$\hookrightarrow \quad 38 ; 5 ; 13: * . p b m=38 ; 5 ; 13: *$.pgm $=38 ; 5 ; 13: * . p p m=38 ; 5 ; 1$ 」

$\hookrightarrow \quad 3: *$. tga $=38 ; 5 ; 13: * . x b m=38 ; 5 ; 13: * . x p m=38 ; 5 ; 13: * . t i$ 」

$\hookrightarrow \mathrm{f}=38 ; 5 ; 13: *$.tiff $=38 ; 5 ; 13: *$.png $=38 ; 5 ; 13: *$.svg $=38$;

$\hookrightarrow \quad 5 ; 13: *$. svgz $=38 ; 5 ; 13: * . m n g=38 ; 5 ; 13: * . p c x=38 ; 5 ; 13:$ 」

$\hookrightarrow \quad$ *.mov $=38 ; 5 ; 13: * . \mathrm{mpg}=38 ; 5 ; 13: * . \mathrm{mpeg}=38 ; 5 ; 13: * . \mathrm{m} 2 \mathrm{v}$ 」

$\hookrightarrow \quad=38 ; 5 ; 13: * . \mathrm{mkv}=38 ; 5 ; 13: *$. webm $=38 ; 5 ; 13: *$. ogm $=38 ; 5$ 」

$\hookrightarrow \quad ; 13: * . \mathrm{mp} 4=38 ; 5 ; 13: * . \mathrm{m} 4 \mathrm{v}=38 ; 5 ; 13: * . \mathrm{mp} 4 \mathrm{v}=38 ; 5 ; 13: *$ 」

$\hookrightarrow \quad$ vob $=38 ; 5 ; 13: *$. qt $=38 ; 5 ; 13: *$.nuv $=38 ; 5 ; 13: *$. wmv $=38$ 」

$\hookrightarrow \quad ; 5 ; 13: *$. asf $=38 ; 5 ; 13: * . r m=38 ; 5 ; 13: * . r m v b=38 ; 5 ; 13:$ 」

$\hookrightarrow \quad * . f l c=38 ; 5 ; 13: *$ avi $=38 ; 5 ; 13: * . f l i=38 ; 5 ; 13: * . f l v=$ ।

$\hookrightarrow \quad 38 ; 5 ; 13: * . g l=38 ; 5 ; 13: * . d l=38 ; 5 ; 13: * . x c f=38 ; 5 ; 13:$ 」

$\hookrightarrow \quad * . x w d=38 ; 5 ; 13: *$. yuv $=38 ; 5 ; 13: *$. cgm $=38 ; 5 ; 13: *$. emf $=$ 」

$\hookrightarrow \quad 38 ; 5 ; 13: *$. axv $=38 ; 5 ; 13: *$. anx $=38 ; 5 ; 13: *$. ogv $=38 ; 5 ; 1$

$\hookrightarrow \quad 3: *$. og $x=38 ; 5 ; 13: *$. aac $=38 ; 5 ; 45: *$. au $=38 ; 5 ; 45: * . f l a$ ।

$\hookrightarrow \quad \mathrm{c}=38 ; 5 ; 45: * . \mathrm{mid}=38 ; 5 ; 45: *$. midi $=38 ; 5 ; 45: * . \mathrm{mka}=38$;

$\hookrightarrow \quad 5 ; 45: *$. mp $3=38 ; 5 ; 45: * . \mathrm{mpc}=38 ; 5 ; 45: *$. ogg $=38 ; 5 ; 45: *$ 」

$\hookrightarrow \quad . r a=38 ; 5 ; 45: *$. wav $=38 ; 5 ; 45: * . a x a=38 ; 5 ; 45: *$. oga $=38$ 」

$\hookrightarrow \quad ; 5 ; 45: *$. spx $=38 ; 5 ; 45: * . x s p f=38 ; 5 ; 45:$

LMOD_sys $=$ Linux

UUFSCELL=kingspeak . peaks

SLURM_STEP_NUM_NODES $=1$

SSH_AUTH_SOCK=/tmp/ssh-bviKxD3bkd/agent. 23093

SRUN_DEBUG $=3$

SLURM_JOBID $=6956232$

_ModuleTable004_=cy9DSFBDLTE4L $0 x p b n V 40$ i 91 dWZzL2NocGM

$\hookrightarrow$ udXRhaC51ZHUvc3lzL21vZHVsZWZpbGVZLONIUEMtMTgvQ29」

$\hookrightarrow \quad$ yZTovdXVmcy9jaHBjLnVOYWguZWR1L3N5cy9pbnNOYWxsZGI」

$\hookrightarrow \quad y L 2 x t b 2 Q v N y 43 L j I 5 L 21 v Z H V s Z W Z p b G V z L 0 N v c m U i L H 0=$

TMOUT $=0$

__LMOD_REF_COUNT__LMFILES_=/uufs/chpc.utah.edu/sys/m 」

$\hookrightarrow$ odulefiles/CHPC-18/Core/chpc/1.0.1ua:1;/uufs/chp」

$\hookrightarrow$ c.utah.edu/sys/modulefiles/CHPC-18/Core/cuda/9.2 」

$\hookrightarrow$.lua: 1 ;/uufs/chpc.utah.edu/sys/modulefiles/CHPC-」

$\hookrightarrow$ 18/Core/gcc/6.4.0.1ua:1

SLURM_NTASKS $=1$

SLURM_LAUNCH_NODE_IPADDR $=10.242 .67 .31$

SLURM_STEP_ID $=0$ 
__LMOD_REF_COUNT_NLSPATH=/uufs/chpc.utah.edu/sys/ins 」

$\hookrightarrow$ talldir/intel/compilers_and_libraries_2018.1.163」

$\hookrightarrow /$ linux/compiler/lib/intel64/locale/\%1_\%t/\%N:2;/u」

$\hookrightarrow$ ufs/chpc.utah.edu/sys/installdir/intel/compilers」

$\hookrightarrow$ _and_libraries_2018.1.163/linux/mkl/lib/intel64_」

$\hookrightarrow \quad$ lin/locale/\%1_\%t/\%N:2;/uufs/chpc.utah.edu/sys/in」

$\hookrightarrow$ stalldir/intel/debugger_2018/gdb/intel64/share/1」

$\hookrightarrow \quad$ ocale/\%1_\%t/\%N:2

_ModuleTable001_=X01vZHVsZVRhYmxlXz17WyJNVHZlcnNpb24」

$\hookrightarrow \quad$ iXTOzLFsiY19yZWJ1aWxkVG1tZSJdPWZhbHNILFsiY19zaG9」

$\hookrightarrow \quad$ ydFRpbWUiXT1mYWxZZSxkZXB0aFQ9e30sZmFtaWx5PXtbIkN

$\hookrightarrow \quad$ vbXBpbGVyIl09ImdjYyIsWyJjdWRhI109ImN1ZGEiLH0sbVQ 」

$\hookrightarrow$ 9e2NocGM9e1siZm4iXT0iL3V1ZnMvY2hwYy51dGFoLmVkdS9 」

$\hookrightarrow$ zeXMvbW9kdWxlZmlsZXMvQ0hQQy0xOC9Db3J1L2NocGMvMS4」

$\hookrightarrow \quad$ wLmx1YSIsWyJmdWxsTmFtZSJdPSJjaHBjLzEuMCIsWyJsb2F 」

$\hookrightarrow$ kT3JkZXIiXT0xLHByb3BUPXt9LFsic3RhY2tEZXB0aCJdPTA 」

$\hookrightarrow \quad$ sWyJzdGFOdXMiXT0iYWN0aXZlII ixbInVzZXJOYW11II09ImN 」

$\hookrightarrow$ ocGMiLH0sY3VkYT17WyJmbiJdPSIvdXVmcy9jaHBjLnV0YWg」

$\hookrightarrow \quad$ uZWR1L3N5cy9tb2R1bGVmaWxlcy9DSFBDLTE4L0NvcmUv

MAIL=/var/spool/mail/USER

PATH=/uufs/chpc. utah. edu/sys/installdir/gcc/6.4.0/bi J

$\hookrightarrow \mathrm{n}$ :/uufs/chpc.utah.edu/sys/installdir/cuda/9.2.14」

$\hookrightarrow$ 8/bin:/usr/lib64/qt-3.3/bin:/usr/kerberos/sbin:/

$\hookrightarrow$ usr/kerberos/bin:/usr/lib64/ccache:/usr/local/bi 」

$\hookrightarrow \mathrm{n}$ :/usr/bin:/usr/local/sbin:/usr/sbin:/uufs/kings」

$\hookrightarrow$ peak.peaks/sys/pkg/slurm/std/bin:/opt/dell/srvad」

$\hookrightarrow \mathrm{min} / \mathrm{bin}$ :/uufs/chpc.utah.edu/common/home/USER/bin

SLURM_TASKS_PER_NODE $=1$

SLURM_STEP_LAUNCHER_PORT $=45416$

SLURM_WORKING_CLUSTER=kingspeak:10.242.67.13:6817:81」

$\hookrightarrow 92$

_=/usr/bin/env

SLURM_JOB_ID $=6956232$

SLURM_STEP_GPUS $=1$

PWD=/uufs/chpc. utah. edu/common/home/USER/brickv2/Aut 」

$\hookrightarrow$ hor-Kit

SLURM_STEPID $=0$

SLURM_JOB_USER=USER

_LMFILES_=/uufs/chpc.utah.edu/sys/modulefiles/CHPC-1」

$\hookrightarrow$ 8/Core/chpc/1.0.lua:/uufs/chpc.utah.edu/sys/modu」

$\hookrightarrow$ lefiles/CHPC-18/Core/cuda/9.2.lua:/uufs/chpc.uta」

$\hookrightarrow$ h.edu/sys/modulefiles/CHPC-18/Core/gcc/6.4.0.lua

SLURM_SRUN_COMM_HOST $=10.242 .67 .31$

CUDA_VISIBLE_DEVICES $=1$

SLURM_CPU_BIND_TYPE=none

LANG=en_US.UTF-8

MODULEPATH=/uufs/chpc. utah. edu/sys/modulefiles/CHPC- 」

$\hookrightarrow$ 18/Compiler/kp/gcc/6.4.0:/uufs/chpc.utah.edu/sys」

$\hookrightarrow$ /modulefiles/CHPC-18/Compiler/gcc/6.4.0:/uufs/ch」

$\hookrightarrow$ pc.utah.edu/sys/modulefiles/CHPC-18/Compiler/cud 」

$\hookrightarrow$ a/9.2:/uufs/chpc.utah.edu/sys/modulefiles/CHPC-1」

$\hookrightarrow$ 8/Linux:/uufs/chpc.utah.edu/sys/modulefiles/CHPC 」

$\hookrightarrow-18 /$ Core:/uufs/chpc.utah.edu/sys/installdir/lmod/」

$\hookrightarrow \quad 7.7 .29 /$ modulefiles/Core

SLURM_UMASK $=0022$

SLURM_PTY_WIN_COL=212
KDEDIRS $=/$ usr

_ModuleTable_Sz_=4

LOADEDMODULES $=\mathrm{chpc} / 1.0: \mathrm{cuda} / 9.2: \mathrm{gcc} / 6.4 .0$

SLURM_JOB_UID $=1080628$

SLURM_NODEID $=0$

SLURM_SUBMIT_DIR=/uufs/chpc.utah.edu/common/home/USE 」

$\hookrightarrow$ R/brickv2/Author-Kit

LMOD_CMD=/uufs/chpc. utah.edu/sys/installdir/lmod/7.7 」

$\hookrightarrow \quad .29 / 1 \mathrm{ibexec} / 1 \mathrm{mod}$

SLURM_NPROCS $=1$

SLURM_TASK_PID $=191863$

SLURM_DISTRIBUTION=cyclic

SLURM_CPUS_ON_NODE=1

SSH_ASKPASS=/usr/libexec/openssh/gnome-ssh-askpass

HISTCONTROL=ignoredups

KRB5CCNAME=KEYRING : persistent : 1080628

SLURM_PROCID $=0$

SLURM_JOB_NODELIST $=\mathrm{kp} 362$

SHLVL=3

HOME=/uufs/chpc. utah.edu/common/home/USER

SLURM_PTY_PORT $=46262$

__LMOD_REF_COUNT_PATH=/uufs/chpc.utah.edu/sys/instal 」

$\hookrightarrow$ ldir/gcc/6.4.0/bin:1;/uufs/chpc.utah.edu/sys/ins」

$\hookrightarrow$ talldir/cuda/9.2.148/bin:1;/usr/lib64/qt-3.3/bin」

$\hookrightarrow \quad: 1 ; /$ usr/kerberos/sbin:1;/usr/kerberos/bin:1;/usr 」

$\hookrightarrow$ /lib64/ccache:1;/usr/local/bin:1;/usr/bin:1;/usr 」

$\hookrightarrow /$ local/sbin:1;/usr/sbin:1;/uufs/kingspeak.peaks/」

$\hookrightarrow$ sys/pkg/slurm/std/bin:2;/opt/dell/srvadmin/bin: 1

$\hookrightarrow \quad ; /$ uufs/chpc.utah.edu/common/home/USER/bin:2

SLURM_LOCALID $=0$

_ModuleTable002_=Y3VkYS85LjIubHVhI ixbImZ1bGx0YW11Ilo,

$\hookrightarrow$ 9ImN1ZGEvOS4yIixbImxvYWRPcmRlciJdPTIscHJvcFQ9e2F

$\hookrightarrow \quad$ yY2g9e1siZ3B1I109MSx9LH0sWyJzdGFja0RlcHRoIl09MCX 」

$\hookrightarrow$ bInNOYXR1cyJdPSJhY3RpdmUiLFsidXN1ck5hbWUiXT0iY3V」

$\hookrightarrow \quad$ kYS85LjIiLH0sZ2NjPXtbImZuI109Ii91dWZzL2NocGMudXR 」

$\hookrightarrow$ haC51ZHUvc31zL21vZHVsZWZpbGVZLONIUEMtMTgvQ29yZS9」

$\hookrightarrow$ nY2MvNi40LjAubHVhIixbImZ1bGx0YW11Il09ImdjYy82LjQ 」

$\hookrightarrow \quad$ UMCIsWyJsb2FkT3JkZXIiXTOzLHByb3BUPXt9LFsic3RhY2t

$\hookrightarrow$ EZXB0aCJdPTAsWyJzdGF0dXMiXT0i YWN0aXZlIixbInVzZXJ

$\hookrightarrow \quad$ OYW11Il09ImdjYy82LjQuMCIsfSx9LG1wYXRoQT17Ii 191dWZ ।

$\hookrightarrow \quad$ zL2NocGMudXRhaC51ZHUvc3lzL21vZHVsZWZpbGVzL0NI

SLURM_CLUSTER_NAME=kingspeak

SLURM_JOB_CPUS_PER_NODE $=1$

SLURM_JOB_GID $=2030$

SLURM_SUBMIT_HOST=kingspeak1

SLURM_GTIDS $=0$

GCC_LIB=/uufs/chpc. utah. edu/sys/installdir/gcc/6.4.0, $\hookrightarrow \quad / 1$ ib64

__LMOD_REF_COUNT_INCLUDE=/uufs/chpc.utah.edu/sys/ins 」

$\hookrightarrow$ talldir/gcc/6.4.0/include:1

BASH_ENV=/uufs/chpc.utah. edu/sys/installdir/lmod/7.7 」

$\hookrightarrow .29 /$ init/bash

SLURM_JOB_PARTITION=soc-gpu-kp

MODULERCFILE=/uufs/chpc. utah. edu/sys/modulefiles/etc 」

$\hookrightarrow \quad / \mathrm{rC}$

LOGNAME $=$ USER 
QTLIB=/usr/lib64/qt-3.3/lib

CVS_RSH=ssh

SLURM_STEP_NUM_TASKS $=1$

BASHRC_LOADED $=1$

XDG_DATA_DIRS=/uufs/chpc. utah.edu/common/home/USER/. 」

$\hookrightarrow$ local/share/flatpak/exports/share:/var/lib/flatp」

$\hookrightarrow$ ak/exports/share:/usr/local/share:/usr/share

SSH_CONNECTION=155.98.69.105 45432 155.101.26.20 22

SLURM_JOB_ACCOUNT $=$ ACCT

GPU_DEVICE_ORDINAL $=1$

MODULESHOME=/uufs/chpc. utah. edu/sys/installdir/lmod/ 」

$\hookrightarrow \quad 7.7 .29$

SLURM_JOB_NUM_NODES $=1$

__LMOD_REF_COUNT_LD_LIBRARY_PATH=/uufs/chpc.utah.edu 」

$\hookrightarrow$ /sys/installdir/gcc/6.4.0/lib64:1;/uufs/chpc.uta

$\hookrightarrow$ h.edu/sys/installdir/gcc/6.4.0/lib:1;/uufs/chpc. 」

$\hookrightarrow$ utah.edu/sys/installdir/cuda/9.2.148/lib64:1;/uu」

$\hookrightarrow \mathrm{fs} / \mathrm{kingspeak}$. peaks/sys/pkg/slurm/std/lib:2

LESSOPEN $=|| /$ usr $/$ bin/lesspipe. sh \%s

LMOD_SETTARG_FULL_SUPPORT=no

_-_Init_Default_Modules $=1$

SLURM_STEP_TASKS_PER_NODE=1

LMOD_FAMILY_COMPILER $=\mathrm{gCC}$

SLURM_STEP_NODELIST $=\mathrm{kp} 362$

XDG_RUNTIME_DIR=/run/user $/ 1080628$

QT_PLUGIN_PATH=/usr/lib64/kde4/plugins: /usr/lib/kde4」

$\hookrightarrow$ /plugins

OSREL=CentOS

__LMOD_REF_COUNT_MANPATH=/uufs/chpc.utah.edu/sys/ins 」

$\hookrightarrow$ talldir/gcc/6.4.0/share/man:1;/uufs/chpc.utah.ed」

$\hookrightarrow$ u/sys/installdir/lmod/7.7.29/share/man:1;/usr/ke」

$\hookrightarrow$ rberos/man:1;/usr/local/share/man:1;/usr/share/m」

$\hookrightarrow$ an/overrides:1;/usr/share/man: 1;/uufs/kingspeak. 」

$\hookrightarrow$ peaks/sys/pkg/slurm/std/share/man: 3

LMOD_DIR=/uufs/chpc.utah.edu/sys/installdir/lmod/7.7」

$\hookrightarrow \quad .29 /$ libexec

SLURM_CPU_BIND=quiet, none

INCLUDE=/uufs/chpc. utah. edu/sys/installdir/gcc/6.4.0 」

$\hookrightarrow$ /include

BASH_FUNC_module ()$=()$ \{ eval $\$\left(\$ L M O D \_C M D\right.$ bash " $\$ Q$ ")

$\hookrightarrow \quad$ \&\& eval $\$\left(\$\left\{L M O D \_S E T T A R G \_C M D:-:\right\}-s\right.$ sh)

\}

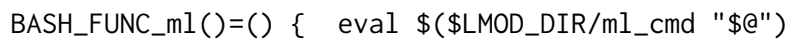

\}

+ lsb_release -a

LSB Version: :core-4.1-amd64:core-4.1-noarch: 」

$\hookrightarrow \quad c x x-4.1-a m d 64: c x x-4.1-n o a r c h:$ desktop-4.1-amd64:d」

$\hookrightarrow$ esktop-4.1-noarch: languages-4.1-amd64: languages- 」

$\hookrightarrow$ 4.1-noarch:printing-4.1-amd64:printing-4.1-noarch

Distributor ID: Centos

Description: CentOS Linux release 7.6.1810

$\hookrightarrow$ (Core)

Release:

Codename:

7.6.1810

+ uname $-a$
Linux kp362 3.10.0-957.1.3.el7.x86_64 \#1 SMP Thu Nov

$\hookrightarrow \quad 2914: 49: 43$ UTC $2018 \times 86 \_64 \times 86 \_64 \times 86 \_64$

$\hookrightarrow$ GNU/Linux

+ lscpu

Architecture:

x86_64

CPU op-mode(s): $\quad 32-$ bit, 64-bit

Byte Order:

$\mathrm{CPU}(\mathrm{s})$ :

Little Endian

On-line CPU(s) list: $0-55$

Thread(s) per core: 2

Core(s) per socket: 14

Socket (s): 2

NUMA node(s): $\quad 2$

Vendor ID: GenuineIntel

CPU family: 6

Model: $\quad 79$

Model name: Intel(R) Xeon(R) CPU E5-2680 v4

$\hookrightarrow$ a $2.40 \mathrm{GHz}$

Stepping: 1

CPU MHz: $\quad 3277.001$

CPU $\max \mathrm{MHz}: \quad 3300.0000$

CPU min MHz: $\quad 1200.0000$

BogoMIPS: $\quad 4799.66$

Virtualization: $\quad \mathrm{VT}-\mathrm{x}$

L1d cache: $\quad 32 \mathrm{~K}$

L1i cache: $\quad 32 \mathrm{~K}$

L2 cache: $\quad 256 \mathrm{~K}$

L3 cache: $\quad 35840 \mathrm{~K}$

NUMA node $0 \mathrm{CPU}(\mathrm{s}): \quad 0,2,4,6,8,10,12,14,16,18,20,22$ 」

$\hookrightarrow \quad, 24,26,28,30,32,34,36,38,40,42,44,46,48,50,52,54$

NUMA node1 CPU(s): $\quad 1,3,5,7,9,11,13,15,17,19,21,23$ 」

$\hookrightarrow \quad, 25,27,29,31,33,35,37,39,41,43,45,47,49,51,53,55$

Flags: fpu vme de pse tsc msr pae mce

$\hookrightarrow$ cx8 apic sep mtrr pge mca cmov pat pse36 clflush

$\hookrightarrow$ dts acpi mmx fxsr sse sse2 ss ht tm pbe syscall $n x$

$\hookrightarrow$ pdpe1gb rdtscp $1 \mathrm{~m}$ constant_tsc arch_perfmon pebs

$\hookrightarrow$ bts rep_good nopl xtopology nonstop_tsc aperfmperf

$\hookrightarrow$ eagerfpu pni pclmulqdq dtes64 monitor ds_cpl vmx

$\hookrightarrow$ smx est tm2 ssse3 sdbg fma cx16 xtpr pdcm pcid dca

$\hookrightarrow$ sse4_1 sse4_2 x2apic movbe popcnt aes xsave avx

$\hookrightarrow \quad$ f16c rdrand lahf_lm abm 3dnowprefetch epb cat_13

$\hookrightarrow$ cdp_13 intel_pt tpr_shadow vnmi flexpriority ept

$\hookrightarrow$ vpid fsgsbase tsc_adjust bmi1 hle avx2 smep bmi2

$\hookrightarrow$ erms invpcid rtm cqm rdt_a rdseed adx smap

$\hookrightarrow \quad x$ saveopt cqm_llc cqm_occup_llc cqm_mbm_total

$\hookrightarrow$ cqm_mbm_local dtherm ida arat pln pts

+ cat /proc/meminfo

MemTotal: $\quad 264033424 \mathrm{kB}$

MemFree: $\quad 241238380 \mathrm{kB}$

MemAvailable: $239329644 \mathrm{kB}$

Buffers: $0 \mathrm{kB}$

Cached: $\quad 19087192 \mathrm{kB}$

SwapCached: $\quad 0 \mathrm{kB}$

Active: $\quad 978212 \mathrm{kB}$

Inactive: $\quad 19121056 \mathrm{kB}$

Active(anon): $\quad 900108 \mathrm{kB}$ 
Exploiting Reuse and Vectorization in Blocked Stencil Computations on CPUs and GPUs

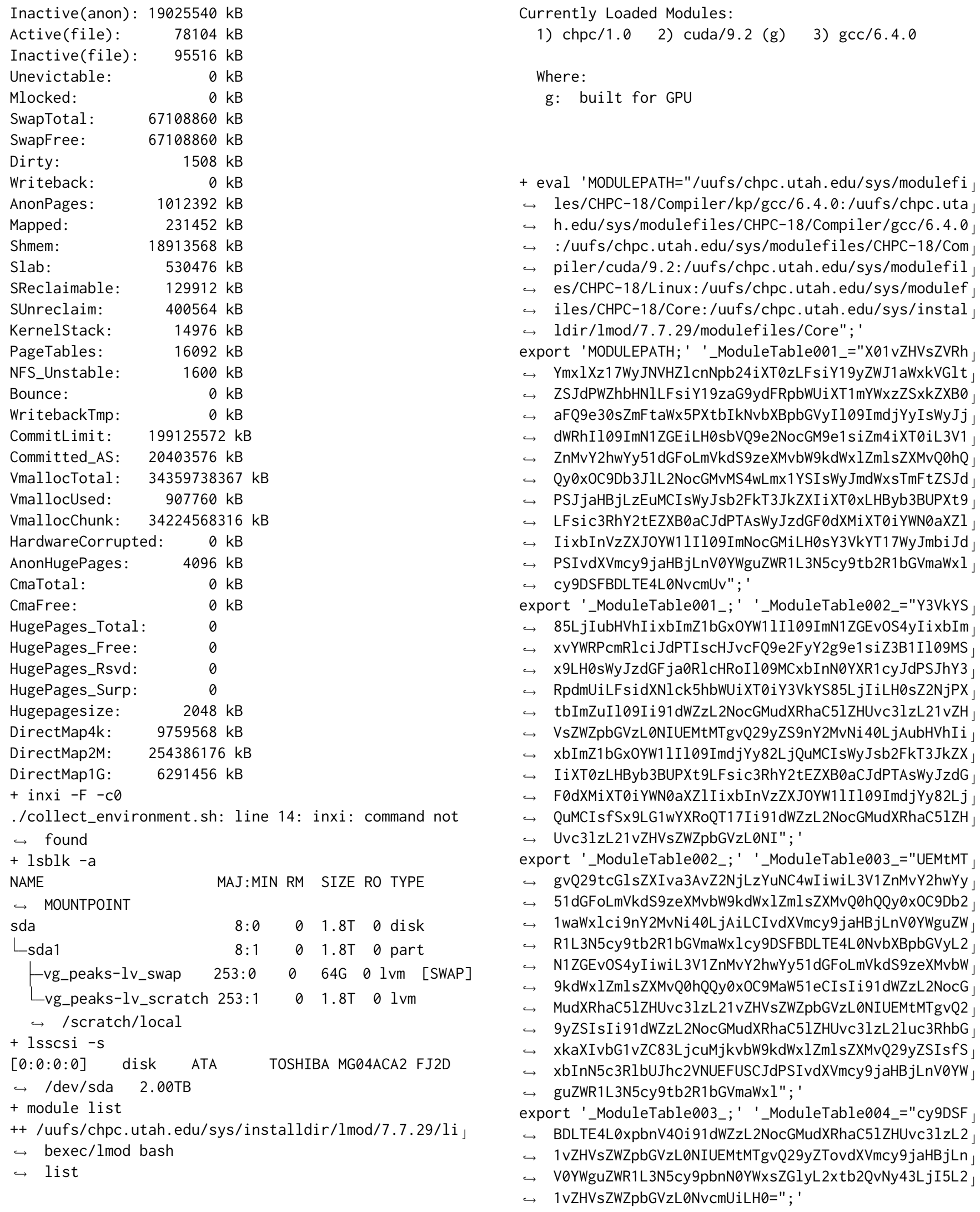


export '_ModuleTable004_; '_ModuleTable_Sz_="4"; export '_ModuleTable_Sz_;'

++ MODULEPATH=/uufs/chpc.utah.edu/sys/modulefiles $/ \mathrm{CH}_{\rfloor}$ $\hookrightarrow \mathrm{PC}-18 /$ Compiler/kp/gcc/6.4.0:/uufs/chpc.utah.edu/」 $\hookrightarrow$ sys/modulefiles/CHPC-18/Compiler/gcc/6.4.0:/uufs」 $\hookrightarrow$ /chpc.utah.edu/sys/modulefiles/CHPC-18/Compiler/」 $\hookrightarrow$ cuda/9.2:/uufs/chpc.utah.edu/sys/modulefiles/CHP」 $\hookrightarrow$ C-18/Linux:/uufs/chpc.utah.edu/sys/modulefiles/C J $\hookrightarrow$ HPC-18/Core:/uufs/chpc.utah.edu/sys/installdir/l」 $\hookrightarrow \mathrm{mod} / 7.7 .29 / \mathrm{modulefiles} /$ Core

$++$

export MODULEPATH

++_ModuleTable001_=X01vZHVsZVRhYmxlXz17WyJNVHZlcnNp

$\hookrightarrow$ b24iXT0zLFsiY19yZWJ1aWxkVGltZSJdPWZhbHN1LFsiY19z」

$\hookrightarrow \quad$ aG9ydFRpbWUiXT1mYWxzZSxkZXB0aFQ9e30sZmFtaWx5PXtb」

$\hookrightarrow \quad$ IkNvbXBpbGVyI109ImdjYyIsWyJjdWRhII09ImN1ZGEiLH0s 」

$\hookrightarrow$ bVQ9e2NocGM9e1siZm4iXT0iL3V1ZnMvY2hwYy51dGFoLmVk 」

$\hookrightarrow$ dS9zeXMvbW9kdWxlZmlsZXMvQ0hQQy0xOC9Db3J1L2NocGMv 」

$\hookrightarrow$ MS4wLmx1YSIsWyJmdWxsTmFtZSJdPSJjaHBjLzEuMCIsWyJs 」

$\hookrightarrow$ b2FkT3JkZXIiXT0xLHByb3BUPXt9LFsic3RhY2tEZXBOaCJd」

$\hookrightarrow$ PTAsWyJzdGF0dXMiXT0iYWN0aXZlIixbInVzZXJOYW11Il09」

$\hookrightarrow \quad$ ImNocGMiLH0sY3VkYT17WyJmbi JdPSIvdXVmcy9jaHBjLnV0,

$\hookrightarrow \quad$ YWguZWR1L3N5cy9tb2R1bGVmaWxlcy9DSFBDLTE4LONvcmUv

$++$

export_ModuleTable001_

++_ModuleTable002_=Y3VkYS85LjIubHVhIixbImZ1bGx0YW11

$\hookrightarrow \quad$ Il09ImN1ZGEv0S4yIixbImxvYWRPcmRlci JdPTIscHJvcFQ9 」

$\hookrightarrow$ e2FyY2g9e1siZ3B1I109MSx9LH0sWyJzdGFja0RlcHRoIl09」

$\hookrightarrow \quad$ MCXbInNOYXR1cyJdPSJhY3RpdmUiLFsidXNlck5hbWUiXT0i 」

$\hookrightarrow \quad$ Y3VkYS85LjIiLH0sZ2NjPXtbImZuI109Ii91dWZzL2NocGMu」

$\hookrightarrow$ dXRhaC51ZHUvc3lzL21vZHVsZWZpbGVzLONIUEMtMTgvQ29y 」

$\hookrightarrow$ ZS9nY2MvNi40LjAubHVhIixbImZ1bGxOYW11Il09ImdjYy82 」

$\hookrightarrow$ LjQuMCIsWyJsb2FkT3JkZXIiXTOzLHByb3BUPXt9LFsic3Rh」

$\hookrightarrow$ Y2tEZXB0aCJdPTAsWyJzdGFOdXMiXT0iYWNOaXZlIixbInVz

$\hookrightarrow \quad$ ZXJOYW11I109ImdjYy82LjQUMCIsfSX9LG1wYXRoQT17Ii91 」

$\hookrightarrow$ dWZzL2NocGMudXRhaC51ZHUvc3lzL21vZHVsZWZpbGVLONI

$++$

export_ModuleTable002

++ _ModuleTable003_=UEMtMTgvQ29tcGlsZXIva3AvZ2NjLzYu」

$\hookrightarrow \quad$ NC4wIiwiL3V1ZnMvY2hwYy51dGFoLmVkdS9zeXMvbW9kdWxl」

$\hookrightarrow \quad$ ZmlsZXMvQ0hQQy0xOC9Db21waWxlci9nY2MvNi40LjAiLCIv」

$\hookrightarrow$ dXVmcy9jaHBjLnV0YWguZWR1L3N5cy9tb2R1bGVmaWxlcy9D 」

$\hookrightarrow \quad$ SFBDLTE4LONvbXBpbGVyL2N1ZGEvOS4yI iwiL3V1ZnMvY2hw 」

$\hookrightarrow \quad$ Yy51dGFoLmVkdS9zeXMvbW9kdWxlZmlsZXMvQ0hQQy0x0C9M」

$\hookrightarrow \quad$ aW51eCIsIi91dWZzL2NocGMudXRhaC51ZHUvc3lzL21vZHVs 」

$\hookrightarrow \quad$ ZWZpbGVZL0NIUEMtMTgVQ29yZSIsIi91dWZzL2NocGMudXRh」

$\hookrightarrow \quad$ CC51ZHUvc3lzL2luc3RhbGxkaXIvbG1vZC83LjcuMjkvbW9k 」

$\hookrightarrow \quad d W x 1 Z m l s Z X M v Q 29 y Z S I s f S x b I n N 5 c 3 R I b U J h c 2 V N U E F U S C J d$ 」

$\hookrightarrow$ PSIvdXVmcy9jaHBjLnV0YWguZWR1L3N5cy9tb2R1bGVmaWxl

$++$

export_ModuleTable003_

++_ModuleTable004_=cy9DSFBDLTE4LOxpbnV40i91dWZzL2No 」

$\hookrightarrow \quad$ cGMudXRhaC51ZHUvc3lzL21vZHVsZWZpbGVzLONIUEMtMTgv 」

$\hookrightarrow$ Q29yZTovdXVmcy9jaHBjLnV0YWguZWR1L3N5cy9pbnNOYWXs 」

$\hookrightarrow$ ZGlyL2xtb2QvNy43LjI5L21vZHVsZWZpbGVzLONvcmUiLH0=

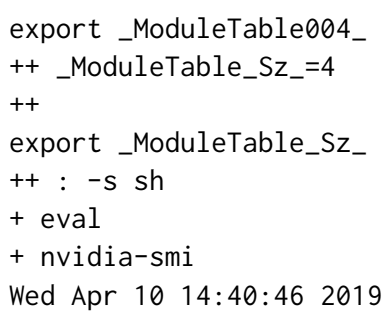

system

bus
Computer Motherboard 
Exploiting Reuse and Vectorization in Blocked Stencil Computations on CPUs and GPUs




10/1a generic Xeon E7 $\hookrightarrow \quad v 4 /$ Xeon E5 v4/Xeon E3 v4/Xeon D Caching Agent $10 / 1 \mathrm{~b} \quad$ generic Xeon E7 $\hookrightarrow \quad v 4 /$ Xeon E5 v4/Xeon E3 v4/Xeon D Caching Agent 10/1c generic Xeon E7 $\hookrightarrow \quad v 4 /$ Xeon E5 v4/Xeon E3 v4/Xeon D Caching Agent /0/1d generic Xeon E7 $\hookrightarrow \quad v 4 /$ Xeon E5 v4/Xeon E3 v4/Xeon D Caching Agent 10/1e generic Xeon E7 $\hookrightarrow \quad v 4 /$ Xeon E5 v4/Xeon E3 v4/Xeon D Caching Agent 10/1f generic Xeon E7 $\hookrightarrow \quad v 4 /$ Xeon E5 v4/Xeon E3 v4/Xeon D Caching Agent $10 / 20 \quad$ generic Xeon E7 $\hookrightarrow \quad v 4 /$ Xeon E5 v4/Xeon E3 v4/Xeon D Caching Agent 10/21 generic Xeon E7 $\hookrightarrow \quad v 4 /$ Xeon E5 v4/Xeon E3 v4/Xeon D Caching Agent 10/22 generic Xeon E7 $\hookrightarrow \quad v 4 /$ Xeon E5 v4/Xeon E3 v4/Xeon D Caching Agent /0/23 generic Xeon E7 $\hookrightarrow \quad v 4 /$ Xeon E5 v4/Xeon E3 v4/Xeon D Caching Agent 10/24 generic Xeon E7 $\hookrightarrow \quad v 4 /$ Xeon E5 v4/Xeon E3 v4/Xeon D Caching Agent $10 / 25$ generic Xeon E7 $\hookrightarrow \quad v 4 /$ Xeon E5 v4/Xeon E3 v4/Xeon D Caching Agent 10/26 generic Xeon E7 $\hookrightarrow \quad$ v4/Xeon E5 v4/Xeon E3 v4/Xeon D R2PCIe Agent 10/27 generic Xeon E7 $\hookrightarrow \quad$ v4/Xeon E5 v4/Xeon E3 v4/Xeon D R2PCIe Agent $10 / 28 \quad$ generic Xeon E7 $\hookrightarrow \quad v 4 /$ Xeon E5 v4/Xeon E3 v4/Xeon D Ubox $10 / 29$ generic Xeon E7 $\hookrightarrow \quad v 4 /$ Xeon E5 v4/Xeon E3 v4/Xeon D Ubox $10 / 2$ a generic Xeon E7 $\hookrightarrow \quad v 4 /$ Xeon E5 $v 4 /$ Xeon E3 v4/Xeon D Ubox $10 / 2 \mathrm{~b} \quad$ generic Xeon E7 $\hookrightarrow \quad v 4 /$ Xeon E5 $v 4 /$ Xeon E3 v4/Xeon D Home Agent 0 $10 / 2 c \quad$ generic $\quad X e o n E 7$ $\hookrightarrow \quad v 4 /$ Xeon E5 v4/Xeon E3 $v 4 /$ Xeon D Home Agent 0 $10 / 2 d \quad$ generic Xeon E7 $\hookrightarrow \quad v 4 /$ Xeon E5 v4/Xeon E3 $v 4 /$ Xeon D Home Agent $\theta$ Debug 10/2e generic Xeon E7 $\hookrightarrow \quad v 4 /$ Xeon E5 $v 4 /$ Xeon E3 $v 4 /$ Xeon D Home Agent 1 $10 / 2 \mathrm{f}$ generic Xeon E7 $\hookrightarrow \quad v 4 /$ Xeon E5 $v 4 /$ Xeon E3 $v 4 /$ Xeon $D$ Home Agent 1 $10 / 30 \quad$ generic Xeon E7 $\hookrightarrow \quad v 4 /$ Xeon E5 v4/Xeon E3 v4/Xeon D Home Agent 1 Debug 10/31 generic Xeon E7 $\hookrightarrow \quad v 4 /$ Xeon E5 v4/Xeon E3 v4/Xeon D Memory Controller $\hookrightarrow 0$ - Target Address/Thermal/RAS

10/32 generic Xeon E7

$\hookrightarrow \quad$ v4/Xeon E5 v4/Xeon E3 v4/Xeon D Memory Controller $\hookrightarrow 0$ - Target Address/Thermal/RAS
10/33 generic Xeon E7 $\hookrightarrow \quad v 4 /$ Xeon E5 v4/Xeon E3 v4/Xeon D Memory Controller $\hookrightarrow 0$ - Channel Target Address Decoder 10/34 generic Xeon E7 $\hookrightarrow \quad$ v4/Xeon E5 v4/Xeon E3 v4/Xeon D Memory Controller $\hookrightarrow \quad$ - Channel Target Address Decoder

10/35 generic Xeon E7

$\hookrightarrow \quad v 4 /$ Xeon E5 v4/Xeon E3 v4/Xeon D DDRIO Channel 0/1

$\hookrightarrow$ Broadcast

10/36 generic Xeon E7

$\hookrightarrow \quad$ v4/Xeon E5 v4/Xeon E3 v4/Xeon D DDRIO Global

$\hookrightarrow$ Broadcast

10/37 generic Xeon E7

$\hookrightarrow \quad v 4 /$ Xeon E5 v4/Xeon E3 v4/Xeon D Memory Controller

$\hookrightarrow \quad 0$ - Channel 0 Thermal Control

10/38 generic Xeon E7

$\hookrightarrow \quad v 4 /$ Xeon E5 v4/Xeon E3 v4/Xeon D Memory Controller

$\hookrightarrow \quad 0$ - Channel 1 Thermal Control

10/39 generic Xeon E7

$\hookrightarrow \quad$ v4/Xeon E5 v4/Xeon E3 v4/Xeon D Memory Controller

$\hookrightarrow \quad 0$ - Channel 0 Error

10/3a generic Xeon E7

$\hookrightarrow \quad v 4 /$ Xeon E5 v4/Xeon E3 v4/Xeon D Memory Controller

$\hookrightarrow \quad 0$ - Channel 1 Error

10/3b generic Xeon E7

$\hookrightarrow \quad v 4 /$ Xeon E5 v4/Xeon E3 v4/Xeon D DDRIO Channel 0/1

$\hookrightarrow$ Interface

10/3c generic Xeon E7

$\hookrightarrow \quad$ v4/Xeon E5 v4/Xeon E3 v4/Xeon D DDRIO Channel 0/1

$\hookrightarrow$ Interface

10/3d generic Xeon E7

$\hookrightarrow \quad$ v4/Xeon E5 v4/Xeon E3 v4/Xeon D DDRIO Channel $0 / 1$

$\hookrightarrow$ Interface

$10 / 3$ e generic Xeon E7

$\hookrightarrow \quad v 4 /$ Xeon E5 v4/Xeon E3 v4/Xeon D DDRIO Channel $0 / 1$

$\hookrightarrow$ Interface

10/3f generic Xeon E7

$\hookrightarrow \quad v 4 /$ Xeon E5 v4/Xeon E3 v4/Xeon D Target

$\hookrightarrow$ Address/Thermal/RAS

10/40 generic Xeon E7

$\hookrightarrow \quad$ v4/Xeon E5 v4/Xeon E3 v4/Xeon D Target

$\hookrightarrow$ Address/Thermal/RAS

10/41 generic Xeon E7

$\hookrightarrow \quad$ v4/Xeon E5 v4/Xeon E3 v4/Xeon D Channel Target

$\hookrightarrow$ Address Decoder

10/42 generic Xeon E7

$\hookrightarrow \quad$ v4/Xeon E5 v4/Xeon E3 v4/Xeon D Channel Target

$\hookrightarrow$ Address Decoder

10/43 generic Xeon E7

$\hookrightarrow \quad$ v4/Xeon E5 v4/Xeon E3 v4/Xeon D DDRIO Channel 2/3

$\hookrightarrow$ Broadcast

10/44 generic Xeon E7

$\hookrightarrow \quad$ v4/Xeon E5 v4/Xeon E3 v4/Xeon D DDRIO Global

$\hookrightarrow$ Broadcast

10/45 generic Xeon E7

$\hookrightarrow \quad v 4 /$ Xeon E5 v4/Xeon E3 v4/Xeon D Memory Controller

$\hookrightarrow 1$ - Channel 0 Thermal Control 
Exploiting Reuse and Vectorization in Blocked Stencil Computations on CPUs and GPUs

$\begin{array}{lcc}\text { /0/46 } & \text { generic } & \text { Xeon E7 } \\ \hookrightarrow \quad \text { v4/Xeon E5 v4/Xeon E3 v4/Xeon D Memory Controller }\end{array}$

$\hookrightarrow 1$ - Channel 1 Thermal Control

10/47 generic Xeon E7

$\hookrightarrow \quad$ v4/Xeon E5 v4/Xeon E3 v4/Xeon D Memory Controller

$\hookrightarrow 1$ - Channel 0 Error

$10 / 48 \quad$ generic Xeon E7

$\hookrightarrow \quad$ v4/Xeon E5 v4/Xeon E3 v4/Xeon D Memory Controller

$\hookrightarrow 1$ - Channel 1 Error

10/49 generic Xeon E7

$\hookrightarrow \quad$ v4/Xeon E5 v4/Xeon E3 v4/Xeon D DDRIO Channel 2/3

$\hookrightarrow$ Interface

$10 / 4 a \quad$ generic Xeon E7

$\hookrightarrow \quad$ v4/Xeon E5 v4/Xeon E3 v4/Xeon D DDRIO Channel 2/3

$\hookrightarrow$ Interface

$10 / 4 b \quad$ generic Xeon E7

$\hookrightarrow \quad$ v4/Xeon E5 v4/Xeon E3 v4/Xeon D DDRIO Channel 2/3

$\hookrightarrow$ Interface

$10 / 4 c \quad$ generic Xeon E7

$\hookrightarrow \quad$ v4/Xeon E5 v4/Xeon E3 v4/Xeon D DDRIO Channel 2/3

$\hookrightarrow$ Interface

10/4d generic Xeon E7

$\hookrightarrow \quad v 4 /$ Xeon E5 v4/Xeon E3 v4/Xeon D Power Control Unit

$10 / 4 \mathrm{e} \quad$ generic Xeon E7

$\hookrightarrow \quad v 4 /$ Xeon E5 v4/Xeon E3 v4/Xeon D Power Control Unit

$10 / 4 f \quad$ generic Xeon E7

$\hookrightarrow \quad v 4 /$ Xeon E5 v4/Xeon E3 v4/Xeon D Power Control Unit

$10 / 50 \quad$ generic Xeon E7

$\hookrightarrow \quad v 4 /$ Xeon E5 v4/Xeon E3 v4/Xeon D Power Control Unit

/0/51 generic Xeon E7

$\hookrightarrow \quad v 4 /$ Xeon E5 v4/Xeon E3 v4/Xeon D Power Control Unit

$10 / 52 \quad$ generic Xeon E7

$\hookrightarrow \quad v 4 /$ Xeon E5 v4/Xeon E3 v4/Xeon D Power Control Unit

$10 / 53 \quad$ generic Xeon E7

$\hookrightarrow \quad$ v4/Xeon E5 v4/Xeon E3 v4/Xeon D Power Control Unit

$10 / 1 \quad$ bridge Xeon E7

$\hookrightarrow \quad$ v4/Xeon E5 v4/Xeon E3 v4/Xeon D PCI Express Root

$\hookrightarrow$ Port 1

$10 / 2 \quad$ bridge Xeon E7

$\hookrightarrow \quad v 4 /$ Xeon E5 v4/Xeon E3 v4/Xeon D PCI Express Root

$\hookrightarrow$ Port 2

$10 / 2 / 0$ display GP100GL

$\hookrightarrow \quad$ [Tesla P100 PCIe 16GB]

$10 / 3$ bridge Xeon E7

$\hookrightarrow \quad v 4 /$ Xeon E5 v4/Xeon E3 v4/Xeon D PCI Express Root

$\hookrightarrow$ Port 3

$10 / 3 / 0$ ibo network MT27500

$\hookrightarrow$ Family [ConnectX-3]

10/3.2 bridge Xeon E7

$\hookrightarrow \quad$ v4/Xeon E5 v4/Xeon E3 v4/Xeon D PCI Express Root

$\hookrightarrow$ Port 3

$10 / 5 \quad$ generic Xeon E7

$\hookrightarrow \quad$ v4/Xeon E5 v4/Xeon E3 v4/Xeon D

$\hookrightarrow$ Map/VTd_Misc/System Management

$10 / 5.1 \quad$ generic Xeon E7

$\hookrightarrow \quad$ v4/Xeon E5 v4/Xeon E3 v4/Xeon D IIO Hot Plug
10/5.2 generic Xeon E7

$\hookrightarrow \quad v 4 /$ Xeon E5 v4/Xeon E3 v4/Xeon D IIO RAS/Control

$\hookrightarrow$ Status/Global Errors

10/5.4 generic Xeon E7

$\hookrightarrow \quad v 4 /$ Xeon E5 v4/Xeon E3 v4/Xeon D I/O APIC

10/54 generic Xeon E7

$\hookrightarrow \quad \mathrm{v} 4 /$ Xeon E5 v4/Xeon E3 v4/Xeon D QPI Link 0

10/55 generic Xeon E7

$\hookrightarrow \quad v 4 /$ Xeon E5 $v 4 /$ Xeon E3 $v 4 /$ Xeon D QPI Link 0

10/56 generic Xeon E7

$\hookrightarrow \quad v 4 /$ Xeon E5 v4/Xeon E3 v4/Xeon D QPI Link 0

10/57 generic Xeon E7

$\hookrightarrow \quad v 4 /$ Xeon E5 $v 4 /$ Xeon E3 $v 4 /$ Xeon D QPI Link 1

10/58 generic Xeon E7

$\hookrightarrow \quad v 4 /$ Xeon E5 v4/Xeon E3 v4/Xeon D QPI Link 1

10/59 generic Xeon E7

$\hookrightarrow \quad v 4 /$ Xeon E5 v4/Xeon E3 v4/Xeon D QPI Link 1

10/5a generic Xeon E7

$\hookrightarrow \quad v 4 /$ Xeon E5 v4/Xeon E3 v4/Xeon D R3 QPI Link $0 / 1$

10/5b generic Xeon E7

$\hookrightarrow \quad$ v4/Xeon E5 v4/Xeon E3 v4/Xeon D R3 QPI Link $0 / 1$

$10 / 5 \mathrm{c}$ generic Xeon E7

$\hookrightarrow \quad v 4 /$ Xeon E5 v4/Xeon E3 v4/Xeon D R3 QPI Link $0 / 1$

10/5d generic Xeon E7

$\hookrightarrow \quad v 4 /$ Xeon E5 v4/Xeon E3 v4/Xeon D R3 QPI Link Debug

$10 / 5 \mathrm{e} \quad$ generic Xeon E7

$\hookrightarrow \quad v 4 /$ Xeon E5 v4/Xeon E3 v4/Xeon D Caching Agent

10/5f generic Xeon E7

$\hookrightarrow \quad v 4 /$ Xeon E5 v4/Xeon E3 v4/Xeon D Caching Agent

10/60 generic Xeon E7

$\hookrightarrow \quad v 4 /$ Xeon E5 v4/Xeon E3 v4/Xeon D Caching Agent

/0/61 generic Xeon E7

$\hookrightarrow \quad v 4 /$ Xeon E5 v4/Xeon E3 v4/Xeon D Caching Agent

10/62 generic Xeon E7

$\hookrightarrow \quad v 4 /$ Xeon E5 v4/Xeon E3 v4/Xeon D Caching Agent

10/63 generic Xeon E7

$\hookrightarrow \quad v 4 /$ Xeon E5 $v 4 /$ Xeon E3 $v 4 /$ Xeon D Caching Agent

10/64 generic Xeon E7

$\hookrightarrow \quad v 4 /$ Xeon E5 v4/Xeon E3 v4/Xeon D Caching Agent

10/65 generic Xeon E7

$\hookrightarrow \quad v 4 /$ Xeon E5 v4/Xeon E3 v4/Xeon D Caching Agent

10/66 generic Xeon E7

$\hookrightarrow \quad v 4 /$ Xeon E5 v4/Xeon E3 v4/Xeon D Caching Agent

10/67 generic Xeon E7

$\hookrightarrow \quad v 4 /$ Xeon E5 v4/Xeon E3 v4/Xeon D Caching Agent

10/68 generic Xeon E7

$\hookrightarrow \quad v 4 /$ Xeon E5 v4/Xeon E3 v4/Xeon D Caching Agent

10/69 generic Xeon E7

$\hookrightarrow \quad v 4 /$ Xeon E5 v4/Xeon E3 v4/Xeon D Caching Agent

10/6a generic Xeon E7

$\hookrightarrow \quad v 4 /$ Xeon E5 v4/Xeon E3 v4/Xeon D Caching Agent

/0/6b generic Xeon E7

$\hookrightarrow \quad v 4 /$ Xeon E5 v4/Xeon E3 v4/Xeon D Caching Agent 
$10 / 6 \mathrm{c} \quad$ generic Xeon E7 $\hookrightarrow \quad v 4 /$ Xeon E5 v4/Xeon E3 v4/Xeon D Caching Agent $10 / 6 \mathrm{~d} \quad$ generic Xeon E7 $\hookrightarrow \quad v 4 /$ Xeon E5 v4/Xeon E3 v4/Xeon D Caching Agent $10 / 6$ e generic Xeon E7 $\hookrightarrow \quad v 4 /$ Xeon E5 v4/Xeon E3 v4/Xeon D Caching Agent /0/6f generic Xeon E7 $\hookrightarrow \quad v 4 /$ Xeon E5 v4/Xeon E3 v4/Xeon D Caching Agent 10/70 generic Xeon E7 $\hookrightarrow \quad v 4 /$ Xeon E5 v4/Xeon E3 v4/Xeon D Caching Agent 10/71 generic Xeon E7 $\hookrightarrow \quad v 4 /$ Xeon E5 v4/Xeon E3 v4/Xeon D Caching Agent 10/72 generic Xeon E7 $\hookrightarrow \quad v 4 /$ Xeon E5 v4/Xeon E3 v4/Xeon D Caching Agent 10/73 generic Xeon E7 $\hookrightarrow \quad v 4 /$ Xeon E5 v4/Xeon E3 v4/Xeon D R2PCIe Agent 10/74 generic Xeon E7 $\hookrightarrow \quad v 4 /$ Xeon E5 v4/Xeon E3 v4/Xeon D R2PCIe Agent $10 / 75 \quad$ generic Xeon E7 $\hookrightarrow \quad v 4 /$ Xeon E5 v4/Xeon E3 v4/Xeon D Ubox 10/76 generic Xeon E7 $\hookrightarrow \quad v 4 /$ Xeon E5 $v 4 /$ Xeon E3 v4/Xeon D Ubox /0/77 generic Xeon E7 $\hookrightarrow \quad v 4 /$ Xeon E5 v4/Xeon E3 v4/Xeon D Ubox $10 / 78 \quad$ generic Xeon E7 $\hookrightarrow \quad v 4 /$ Xeon E5 v4/Xeon E3 v4/Xeon D Home Agent 0 10/79 generic Xeon E7 $\hookrightarrow \quad v 4 /$ Xeon E5 $v 4 /$ Xeon E3 $v 4 /$ Xeon $D$ Home Agent 0 10/7a generic Xeon E7 $\hookrightarrow \quad v 4 /$ Xeon E5 v4/Xeon E3 v4/Xeon D Home Agent $\theta$ Debug $10 / 7 \mathrm{~b} \quad$ generic Xeon E7 $\hookrightarrow \quad v 4 /$ Xeon E5 v4/Xeon E3 v4/Xeon D Home Agent 1 10/7c generic Xeon E7 $\hookrightarrow \quad v 4 /$ Xeon E5 $v 4 /$ Xeon E3 $v 4 /$ Xeon D Home Agent 1 10/7d generic Xeon E7 $\hookrightarrow \quad v 4 /$ Xeon E5 v4/Xeon E3 v4/Xeon D Home Agent 1 Debug 10/7e generic Xeon E7 $\hookrightarrow \quad$ v4/Xeon E5 v4/Xeon E3 v4/Xeon D Memory Controller $\hookrightarrow 0$ - Target Address/Thermal/RAS

10/7f generic Xeon E7

$\hookrightarrow \quad$ v4/Xeon E5 v4/Xeon E3 v4/Xeon D Memory Controller

$\hookrightarrow 0$ - Target Address/Thermal/RAS

10/80 generic Xeon E7

$\hookrightarrow \quad$ v4/Xeon E5 v4/Xeon E3 v4/Xeon D Memory Controller

$\hookrightarrow 0$ - Channel Target Address Decoder

10/81 generic Xeon E7

$\hookrightarrow \quad$ v4/Xeon E5 v4/Xeon E3 v4/Xeon D Memory Controller

$\hookrightarrow \quad$ - Channel Target Address Decoder

10/82 generic Xeon E7

$\hookrightarrow \quad v 4 /$ Xeon E5 v4/Xeon E3 v4/Xeon D DDRIO Channel $0 / 1$

$\hookrightarrow$ Broadcast

10/83 generic Xeon E7

$\hookrightarrow \quad v 4 /$ Xeon E5 v4/Xeon E3 v4/Xeon D DDRIO Global

$\hookrightarrow$ Broadcast
10/84 generic Xeon E7 $\hookrightarrow \quad v 4 /$ Xeon E5 v4/Xeon E3 v4/Xeon D Memory Controller $\hookrightarrow \quad 0$ - Channel 0 Thermal Control

10/85 generic Xeon E7

$\hookrightarrow \quad v 4 /$ Xeon E5 v4/Xeon E3 v4/Xeon D Memory Controller

$\hookrightarrow \quad 0$ - Channel 1 Thermal Control

10/86 generic Xeon E7

$\hookrightarrow \quad v 4 /$ Xeon E5 v4/Xeon E3 v4/Xeon D Memory Controller

$\hookrightarrow \quad 0$ - Channel 0 Error

10/87 generic Xeon E7

$\hookrightarrow \quad v 4 /$ Xeon E5 v4/Xeon E3 v4/Xeon D Memory Controller

$\hookrightarrow \quad 0$ - Channel 1 Error

10/88 generic Xeon E7

$\hookrightarrow \quad v 4 /$ Xeon E5 v4/Xeon E3 v4/Xeon D DDRIO Channel $0 / 1$

$\hookrightarrow$ Interface

10/89 generic Xeon E7

$\hookrightarrow \quad v 4 /$ Xeon E5 v4/Xeon E3 v4/Xeon D DDRIO Channel $0 / 1$

$\hookrightarrow$ Interface

10/8a generic Xeon E7

$\hookrightarrow \quad v 4 /$ Xeon E5 v4/Xeon E3 v4/Xeon D DDRIO Channel $0 / 1$

$\hookrightarrow$ Interface

10/8b generic Xeon E7

$\hookrightarrow \quad v 4 /$ Xeon E5 v4/Xeon E3 v4/Xeon D DDRIO Channel $0 / 1$

$\hookrightarrow$ Interface

10/8c generic Xeon E7

$\hookrightarrow \quad v 4 /$ Xeon E5 v4/Xeon E3 v4/Xeon D Target

$\hookrightarrow$ Address/Thermal/RAS

10/8d generic Xeon E7

$\hookrightarrow \quad$ v4/Xeon E5 v4/Xeon E3 v4/Xeon D Target

$\hookrightarrow$ Address/Thermal/RAS

10/8e generic Xeon E7

$\hookrightarrow \quad$ v4/Xeon E5 v4/Xeon E3 v4/Xeon D Channel Target

$\hookrightarrow$ Address Decoder

10/8f generic Xeon E7

$\hookrightarrow \quad v 4 /$ Xeon E5 v4/Xeon E3 v4/Xeon D Channel Target

$\hookrightarrow$ Address Decoder

10/90 generic Xeon E7

$\hookrightarrow$ v4/Xeon E5 v4/Xeon E3 v4/Xeon D DDRIO Channel 2/3

$\hookrightarrow$ Broadcast

/0/91 generic Xeon E7

$\hookrightarrow \quad$ v4/Xeon E5 v4/Xeon E3 v4/Xeon D DDRIO Global

$\hookrightarrow$ Broadcast

10/92 generic Xeon E7

$\hookrightarrow \quad v 4 /$ Xeon E5 v4/Xeon E3 v4/Xeon D Memory Controller

$\hookrightarrow 1$ - Channel 0 Thermal Control

10/93 generic Xeon E7

$\hookrightarrow \quad$ v4/Xeon E5 v4/Xeon E3 v4/Xeon D Memory Controller

$\hookrightarrow \quad 1$ - Channel 1 Thermal Control

10/94 generic Xeon E7

$\hookrightarrow \quad v 4 /$ Xeon E5 v4/Xeon E3 v4/Xeon D Memory Controller

$\hookrightarrow 1$ - Channel 0 Error

10/95 generic Xeon E7

$\hookrightarrow \quad$ v4/Xeon E5 v4/Xeon E3 v4/Xeon D Memory Controller

$\hookrightarrow 1$ - Channel 1 Error

10/96 generic Xeon E7

$\hookrightarrow \quad$ v4/Xeon E5 v4/Xeon E3 v4/Xeon D DDRIO Channel 2/3

$\hookrightarrow$ Interface 
Exploiting Reuse and Vectorization in Blocked Stencil Computations on CPUs and GPUs

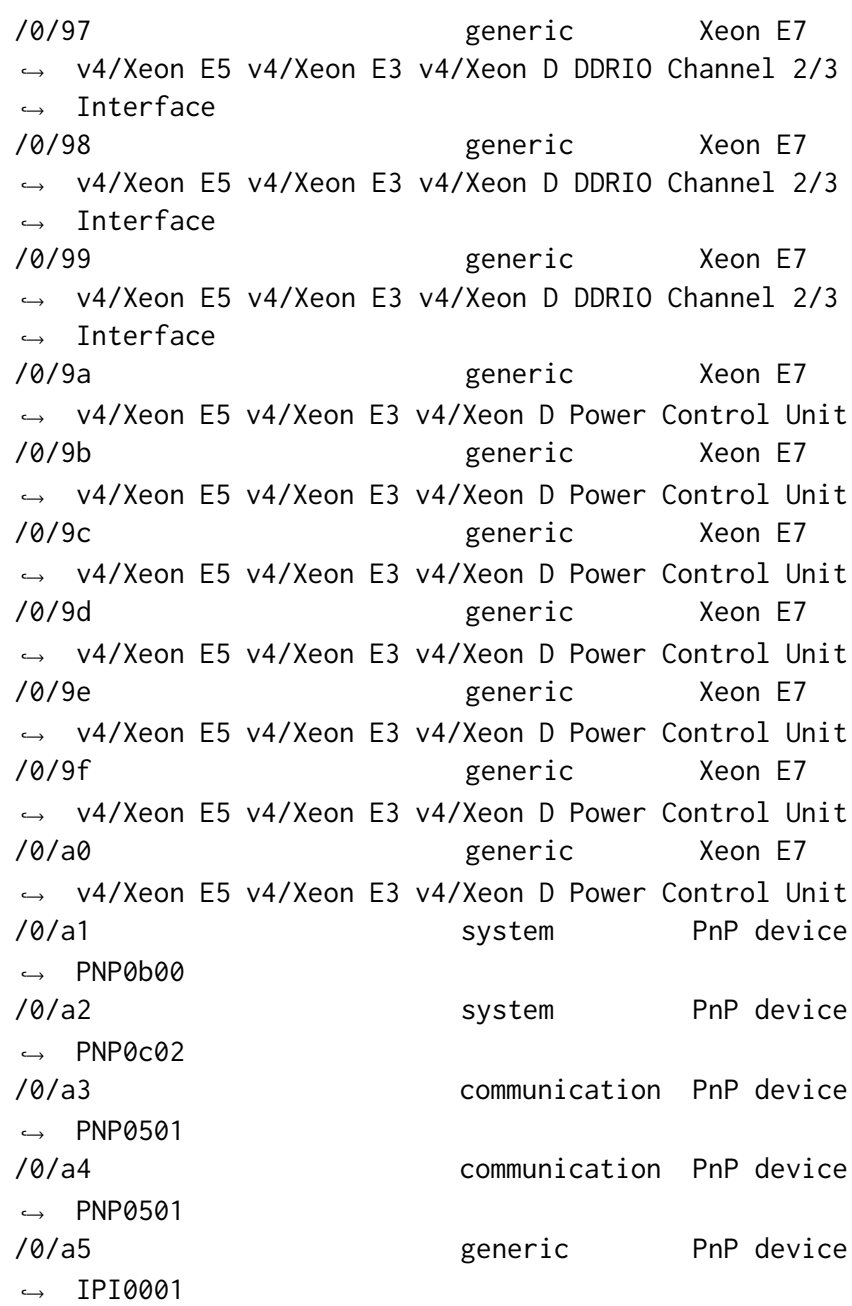

$\hookrightarrow \quad$ IPI0001

WARNING: output may be incomplete or inaccurate, you $\hookrightarrow$ should run this program as super-user.

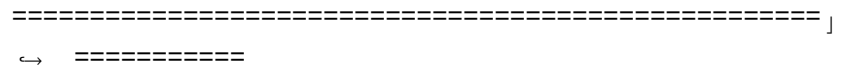

Skylake-X node:

SLURM_NODELIST=notch035

SLURM_CHECKPOINT_IMAGE_DIR=/var/slurm/checkpoint LMOD_FAMILY_COMPILER_VERSION=2019.2.187

MKLROOT=/uufs/chpc . utah. edu/sys/installdir/intel/com 」

$\hookrightarrow$ pilers_and_libraries_2019.2.187/linux/mkl

MANPATH=/uufs/chpc. utah.edu/sys/installdir/intel/doc 」

$\hookrightarrow$ umentation_2019/en/man/common:/uufs/chpc.utah.ed」

$\hookrightarrow$ u/sys/installdir/intel/compilers_and_libraries_2」

$\hookrightarrow$ 019.2.187/linux/mpi/man:/uufs/chpc.utah.edu/sys/」

$\hookrightarrow$ installdir/intel/documentation_2019/en/debugger/।

$\hookrightarrow$ gdb-ia/man:/uufs/chpc.utah.edu/sys/installdir/lm」

$\hookrightarrow$ od/7.7.29/share/man:/usr/kerberos/man:/usr/local」

$\hookrightarrow$ /share/man:/usr/share/man/overrides:/usr/share/m」

$\hookrightarrow$ an:/uufs/notchpeak. peaks/sys/installdir/slurm/st 」

$\hookrightarrow d /$ share/man

SLURM_JOB_NAME=hello
XDG_SESSION_ID $=127512$

HOSTNAME $=$ not $\operatorname{ch} 035$

SLURM_TOPOLOGY_ADDR=notch035

SLURMD_NODENAME=notch035

_ModuleTable003_=cmUiLHOsWyJzeXN0ZW1CYXNITVBBVEgiXT0

$\hookrightarrow \quad$ iL3V1ZnMvY2hwYy51dGFoLmVkdS9zeXMvbW9kdWxlZmlsZXM

$\hookrightarrow \quad$ VQ0hQQy0xOC9MaW51 eDovdXVmcy9jaHBjLnVOYWguZWR1L3N

$\hookrightarrow \quad 5 c y 9 t b 2 R 1 b G V m a W x 1 c y 9 D S F B D L T E 4 L 0 N v c m U 6 L 3 V 1 Z n M v Y 2 h$ 」

$\hookrightarrow \quad$ wYy51dGFoLmVkdS9zeXMvaW5zdGFsbGRpci9sbW9kLzcuNy4」

$\hookrightarrow$ yOS9tb2R1bGVmaWxlcy9Db3J1Iix9

SLURM_PRIO_PROCESS $=0$

SLURM_NODE_ALIASES $=($ null $)$

INTEL_LICENSE_FILE=/uufs/chpc.utah.edu/sys/installdi 」

$\hookrightarrow \quad r /$ intel/licenses

IPPROOT=/uufs/chpc. utah. edu/sys/installdir/intel/com 」

$\hookrightarrow$ pilers_and_libraries_2019.2.187/linux/ipp

__LMOD_REF_COUNT_MODULEPATH=/uufs/chpc.utah.edu/sys /

$\hookrightarrow$ modulefiles/CHPC-18/Compiler/intel/2019.2.187:1;

$\hookrightarrow$ /uufs/chpc.utah.edu/sys/modulefiles/CHPC-18/Linu

$\hookrightarrow \mathrm{x}: 1$;/uufs/chpc.utah.edu/sys/modulefiles/CHPC-18/

$\hookrightarrow$ Core: 1 ;/uufs/chpc.utah.edu/sys/installdir/lmod/7

$\hookrightarrow \quad .7 .29 /$ modulefiles/Core: 1

TERM $=x$ term-256color

SHELL=/bin/bash

HISTSIZE $=1000$

SLURM_JOB_QOS=notchpeak - freecycle

KMP_HOT_TEAMS_MODE $=1$

LMOD_SYSTEM_DEFAULT_MODULES $=c h p c$

MODULEPATH_ROOT=/uufs/chpc.utah.edu/sys/modulefiles/

$\hookrightarrow \quad$ CHPC -18

SSH_CLIENT $=155.97 .232 .2355320022$

SLURM_TOPOLOGY_ADDR_PATTERN=node

TMPDIR $=/ \mathrm{tmp}$

LIBRARY_PATH=/uufs/chpc.utah.edu/sys/installdir/inte

$\hookrightarrow \quad 1 / /$ compilers_and_libraries_2019.2.187/linux/mpi/ .

$\hookrightarrow$ intel64/libfabric/lib:/uufs/chpc.utah.edu/sys/in」

$\hookrightarrow$ stalldir/intel/compilers_and_libraries_2019.2.18」

$\hookrightarrow$ 7/linux/ipp/lib/intel64:/uufs/chpc.utah.edu/sys/」

$\hookrightarrow$ installdir/intel/compilers_and_libraries_2019.2.」

$\hookrightarrow$ 187/linux/compiler/lib/intel64_lin:/uufs/chpc.ut 」

$\hookrightarrow \quad$ ah.edu/sys/installdir/intel/compilers_and_librar

$\hookrightarrow$ ies_2019.2.187/linux/mkl/lib/intel64_lin:/uufs/c

$\hookrightarrow \mathrm{hpc} . u t a h . e d u / s y s /$ installdir/intel/compilers_and_」

$\hookrightarrow$ libraries_2019.2.187/linux/tbb/lib/intel64/gcc4.

$\hookrightarrow 7:$ /uufs/chpc.utah.edu/sys/installdir/intel/compi

$\hookrightarrow$ lers_and_libraries_2019.2.187/linux/tbb/lib/inte

$\hookrightarrow$ 164/gcc4.7:/uufs/chpc.utah.edu/sys/installdir/in」

$\hookrightarrow$ tel/compilers_and_libraries_2019.2.187/linux/daa

$\hookrightarrow$ 1/lib/intel64_lin:/uufs/chpc.utah.edu/sys/instal」

$\hookrightarrow$ ldir/intel/compilers_and_libraries_2019.2.187/li

$\hookrightarrow$ nux/daal/../tbb/lib/intel64_lin/gcc4.4

LMOD_PKG=/uufs/chpc.utah.edu/sys/installdir/lmod/7.7」

$\hookrightarrow \quad .29$

QTDIR=/usr/lib64/qt-3.3

QTINC=/usr/lib64/qt-3.3/include

LMOD_VERSION=7.7.29 
SSH_TTY=/dev/pts $/ 8$

__LMOD_REF_COUNT_LOADEDMODULES=chpc $/ 1.0: 1$; intel $/ 2019$ 」

$\hookrightarrow \quad .2 .187: 1$

QT_GRAPHICSSYSTEM_CHECKED $=1$

OSVER $=7.6 .1810$

USER=USER

\section{SLURM_NNODES $=1$}

LD_LIBRARY_PATH=/uufs/chpc.utah.edu/sys/installdir/g ।

$\hookrightarrow \mathrm{cc} / 8.1 .0 / \mathrm{ib64:/uufs/chpc.utah.edu/sys/installdi}$

$\hookrightarrow \mathrm{r} / \mathrm{gcc} / 8.1 .0 / \mathrm{ib}$ /uufs/chpc.utah.edu/sys/installd

$\hookrightarrow \quad \mathrm{ir} /$ intel/compilers_and_libraries_2019.2.187/linu

$\hookrightarrow \quad x /$ compiler/lib/intel64_lin:/uufs/chpc.utah.edu/s 」

$\hookrightarrow$ ys/installdir/intel//compilers_and_libraries_201」

$\hookrightarrow$ 9.2.187/linux/mpi/intel64/libfabric/lib:/uufs/ch」

$\hookrightarrow$ pc.utah.edu/sys/installdir/intel//compilers_and_-

$\hookrightarrow$ libraries_2019.2.187/linux/mpi/intel64/lib/relea 」

$\hookrightarrow$ se:/uufs/chpc.utah.edu/sys/installdir/intel//com

$\hookrightarrow$ pilers_and_libraries_2019.2.187/linux/mpi/intel6

$\hookrightarrow$ 4/lib:/uufs/chpc.utah.edu/sys/installdir/intel/c 」

$\hookrightarrow$ ompilers_and_libraries_2019.2.187/linux/ipp/lib/

$\hookrightarrow$ intel64:/uufs/chpc.utah.edu/sys/installdir/intel」

$\hookrightarrow$ /compilers_and_libraries_2019.2.187/linux/compil」

$\hookrightarrow$ er/lib/intel64_lin:/uufs/chpc.utah.edu/sys/insta

$\hookrightarrow$ lldir/intel/compilers_and_libraries_2019.2.187/1 」

$\hookrightarrow \quad$ inux/mkl/lib/intel64_lin:/uufs/chpc.utah.edu/sys 」

$\hookrightarrow$ /installdir/intel/compilers_and_libraries_2019.2」

$\hookrightarrow .187 /$ inux/tbb/lib/intel64/gcc4.7:/uufs/chpc.uta

$\hookrightarrow$ h.edu/sys/installdir/intel/compilers_and_librari 」

$\hookrightarrow$ es_2019.2.187/linux/tbb/lib/intel64/gcc4.7:/uufs ।

$\hookrightarrow$ /chpc.utah.edu/sys/installdir/intel/debugger_201」

$\hookrightarrow$ 9/libipt/intel64/lib:/uufs/chpc.utah.edu/sys/ins

$\hookrightarrow$ talldir/intel/compilers_and_libraries_2019.2.187

$\hookrightarrow /$ linux/daal/lib/intel64_lin:/uufs/chpc.utah.edu/ 」

$\hookrightarrow$ sys/installdir/intel/compilers_and_libraries_201」

$\hookrightarrow$ 9.2.187/linux/daal/../tbb/lib/intel64_lin/gcc4.4
LS_COLORS $=r s=0: \mathrm{di}=38 ; 5 ; 27: \ln =38 ; 5 ; 51: \mathrm{mh}=44 ; 38 ; 5 ; 15: \mathrm{p}$ 」

$\hookrightarrow \quad \mathrm{i}=40 ; 38 ; 5 ; 11: \mathrm{so}=38 ; 5 ; 13: \mathrm{do}=38 ; 5 ; 5: \mathrm{bd}=48 ; 5 ; 232 ; 38$ 」

$\hookrightarrow \quad ; 5 ; 11: \mathrm{cd}=48 ; 5 ; 232 ; 38 ; 5 ; 3:$ or $=48 ; 5 ; 232 ; 38 ; 5 ; 9: \mathrm{mi}=0$ 」

$\hookrightarrow \quad 5 ; 48 ; 5 ; 232 ; 38 ; 5 ; 15: \mathrm{su}=48 ; 5 ; 196 ; 38 ; 5 ; 15: \mathrm{sg}=48 ; 5 ; 1$ 」

$\hookrightarrow \quad 1 ; 38 ; 5 ; 16: \mathrm{ca}=48 ; 5 ; 196 ; 38 ; 5 ; 226: \mathrm{tw}=48 ; 5 ; 10 ; 38 ; 5 ; 1$ 」

$\hookrightarrow \quad 6: \mathrm{ow}=48 ; 5 ; 10 ; 38 ; 5 ; 21: \mathrm{st}=48 ; 5 ; 21 ; 38 ; 5 ; 15: \mathrm{ex}=38 ; 5 ;$ 」

$\hookrightarrow \quad 34: * . \operatorname{tar}=38 ; 5 ; 9: * . \operatorname{tgz}=38 ; 5 ; 9: * . \operatorname{arc}=38 ; 5 ; 9: * . \operatorname{arj}=$ 」

$\hookrightarrow \quad 38 ; 5 ; 9: *$. taz $=38 ; 5 ; 9: * .1 \mathrm{ha}=38 ; 5 ; 9: * .1 \mathrm{z} 4=38 ; 5 ; 9: *$.

$\hookrightarrow \quad l z h=38 ; 5 ; 9: * .1 z m a=38 ; 5 ; 9: * . t l z=38 ; 5 ; 9: * . t x z=38 ; 5$ 」

$\hookrightarrow \quad ; 9: * . t z o=38 ; 5 ; 9: * . t 7 z=38 ; 5 ; 9: * . z i p=38 ; 5 ; 9: * . z=38$ ।

$\hookrightarrow \quad ; 5 ; 9: * . z=38 ; 5 ; 9: * . d z=38 ; 5 ; 9: * . g z=38 ; 5 ; 9: * .1 r z=38$ ।

$\hookrightarrow \quad ; 5 ; 9: * .1 z=38 ; 5 ; 9: * .1 z 0=38 ; 5 ; 9: * . x z=38 ; 5 ; 9: * . b z 2=$ 」

$\hookrightarrow \quad 38 ; 5 ; 9: * . b z=38 ; 5 ; 9: * . t b z=38 ; 5 ; 9: * . t b z 2=38 ; 5 ; 9: *$.

$\hookrightarrow \quad \mathrm{tz}=38 ; 5 ; 9: * . \mathrm{deb}=38 ; 5 ; 9: * . \mathrm{rpm}=38 ; 5 ; 9: *$.jar $=38 ; 5 ; 9$ ।

$\hookrightarrow \quad: *$.war $=38 ; 5 ; 9: *$.ear $=38 ; 5 ; 9: *$. sar $=38 ; 5 ; 9: *$. rar $=38$

$\hookrightarrow \quad ; 5 ; 9: *$.alz $=38 ; 5 ; 9: *$. ace $=38 ; 5 ; 9: * . z 00=38 ; 5 ; 9: * . c p$ ।

$\hookrightarrow$ io $=38 ; 5 ; 9: * .7 z=38 ; 5 ; 9: * . r z=38 ; 5 ; 9: * . c a b=38 ; 5 ; 9: *$ 」

$\hookrightarrow \quad$.jpg $=38 ; 5 ; 13: *$.jpeg $=38 ; 5 ; 13: *$.gif $=38 ; 5 ; 13: * . b m p=$ 」

$\hookrightarrow \quad 38 ; 5 ; 13: *$. pbm=38;5;13:*.pgm=38;5;13:*.ppm=38;5;1 」

$\hookrightarrow \quad 3: *$.tga $=38 ; 5 ; 13: * . x b m=38 ; 5 ; 13: * . x p m=38 ; 5 ; 13: *$.ti 」

$\hookrightarrow \mathrm{f}=38 ; 5 ; 13: *$.tiff $=38 ; 5 ; 13: *$.png $=38 ; 5 ; 13: *$.svg $=38$;.

$\hookrightarrow \quad 5 ; 13: *$. svgz $=38 ; 5 ; 13: * . m n g=38 ; 5 ; 13: * . p c x=38 ; 5 ; 13:$ 」

$\hookrightarrow \quad$ *.mov $=38 ; 5 ; 13: * . \mathrm{mpg}=38 ; 5 ; 13: *$.mpeg $=38 ; 5 ; 13: * . \mathrm{m} 2 \mathrm{v}$ ।

$\hookrightarrow \quad=38 ; 5 ; 13: * . m k v=38 ; 5 ; 13: *$. webm $=38 ; 5 ; 13: *$ ogm $=38 ; 5$ 」

$\hookrightarrow \quad ; 13: * . \mathrm{mp} 4=38 ; 5 ; 13: * . \mathrm{m} 4 \mathrm{v}=38 ; 5 ; 13: * . \mathrm{mp} 4 \mathrm{v}=38 ; 5 ; 13: *$ 」

$\hookrightarrow \quad$ vob $=38 ; 5 ; 13: *$.qt $=38 ; 5 ; 13: *$.nuv $=38 ; 5 ; 13: *$. wmv $=38$ 」

$\hookrightarrow \quad ; 5 ; 13: *$. asf $=38 ; 5 ; 13: * . r m=38 ; 5 ; 13: *$.rmvb $=38 ; 5 ; 13:$

$\hookrightarrow \quad * . f l c=38 ; 5 ; 13: *$ avi $=38 ; 5 ; 13: * . f l i=38 ; 5 ; 13: * . f l v=$ ।

$\hookrightarrow \quad 38 ; 5 ; 13: * . g l=38 ; 5 ; 13: * . d l=38 ; 5 ; 13: * . x c f=38 ; 5 ; 13:$ 」

$\hookrightarrow \quad * . x w d=38 ; 5 ; 13: *$. yuv $=38 ; 5 ; 13: *$. cgm $=38 ; 5 ; 13: *$. emf $=$ 」

$\hookrightarrow \quad 38 ; 5 ; 13: *$. axv $=38 ; 5 ; 13: *$. an $x=38 ; 5 ; 13: *$.ogv $=38 ; 5 ; 1$ 」

$\hookrightarrow \quad 3: *$. ogx $=38 ; 5 ; 13: *$. aac $=38 ; 5 ; 45: *$. au $=38 ; 5 ; 45: * . \mathrm{fla}\rfloor$

$\hookrightarrow \mathrm{c}=38 ; 5 ; 45: *$. mid $=38 ; 5 ; 45: *$. midi $=38 ; 5 ; 45: *$. mka=38; ।

$\hookrightarrow \quad 5 ; 45: * . \mathrm{mp} 3=38 ; 5 ; 45: * . \mathrm{mpc}=38 ; 5 ; 45: *$. ogg $=38 ; 5 ; 45: *$ 」

$\hookrightarrow \quad . r a=38 ; 5 ; 45: * . w a v=38 ; 5 ; 45: * . a x a=38 ; 5 ; 45: *$ oga $=38$ 」

$\hookrightarrow \quad ; 5 ; 45: *$. spx $=38 ; 5 ; 45: * . x s p f=38 ; 5 ; 45:$

LMOD_sys $=$ Linux

UUFSCELL=notchpeak . peaks

PSTLROOT=/uufs/chpc. utah. edu/sys/installdir/intel/co 」

$\hookrightarrow$ mpilers_and_libraries_2019.2.187/linux/pstl

FI_PROVIDER_PATH=/uufs/chpc.utah.edu/sys/installdir/ 」

$\hookrightarrow$ intel//compilers_and_libraries_2019.2.187/linux/ 」

$\hookrightarrow \mathrm{mpi} /$ intel64/libfabric/lib/prov

SSH_AUTH_SOCK=/tmp/ssh-ElHXvX90p0/agent. 252968

SLURM_JOBID $=289502$ 
CPATH=/uufs/chpc. utah.edu/sys/installdir/intel/compi 」

$\hookrightarrow$ lers_and_libraries_2019.2.187/linux/ipp/include: 」

$\hookrightarrow$ /uufs/chpc.utah.edu/sys/installdir/intel/compile」

$\hookrightarrow$ rs_and_libraries_2019.2.187/linux/mkl/include:/u」

$\hookrightarrow$ ufs/chpc.utah.edu/sys/installdir/intel/compilers」

$\hookrightarrow$ _and_libraries_2019.2.187/linux/pstl/include:/uu」

$\hookrightarrow$ fs/chpc.utah.edu/sys/installdir/intel/compilers_」

$\hookrightarrow$ and_libraries_2019.2.187/linux/tbb/include:/uufs 」

$\hookrightarrow$ /chpc.utah.edu/sys/installdir/intel/compilers_an」

$\hookrightarrow$ d_libraries_2019.2.187/linux/tbb/include:/uufs/c 」

$\hookrightarrow \mathrm{hpc} . u t a h$. edu/sys/installdir/intel/compilers_and_」

$\hookrightarrow$ libraries_2019.2.187/linux/daal/include

TMOUT $=0$

__LMOD_REF_COUNT__LMFILES_=/uufs/chpc.utah.edu/sys $/ \mathrm{m}$ 」

$\hookrightarrow$ odulefiles/CHPC-18/Core/chpc/1.0.lua: 1 ;/uufs/chp

$\hookrightarrow$ c.utah.edu/sys/modulefiles/CHPC-18/Core/intel/20」

$\hookrightarrow$ 19.2.187.lua:1

KMP_HOT_TEAMS_MAX_LEVEL $=2$

SLURM_NTASKS $=1$

VIRTUAL_ENV=/uufs/chpc. utah.edu/common/home/USER/bri 」

$\hookrightarrow \quad \mathrm{ckv} 2 /$ env

NLSPATH=/uufs/chpc. utah. edu/sys/installdir/intel/com 」

$\hookrightarrow$ pilers_and_libraries_2019.2.187/linux/compiler/1 」

$\hookrightarrow \quad$ ib/intel64/locale/\%1_\%t/\%N:/uufs/chpc.utah.edu/s 」

$\hookrightarrow$ ys/installdir/intel/compilers_and_libraries_2019」

$\hookrightarrow \quad .2 .187 /$ linux/mkl/lib/intel64_lin/locale/\%1_\%t/\%

$\hookrightarrow \quad:$ /uufs/chpc.utah.edu/sys/installdir/intel/debugg」

$\hookrightarrow$ er_2019/gdb/intel64/share/locale/\%1_\%t/\%N

_ModuleTable001_=X01vZHVsZVRhYmxlXz17WyJNVHZlcnNpb24 」

$\hookrightarrow \quad$ iXTOzLFsiY19yZWJ1aWxkVGltZSJdPWZhbHN1LFsiY19zaG9 」

$\hookrightarrow \quad$ ydFRpbWUiXT1mYWxzZSxkZXB0aFQ9e30sZmFtaWx5PXtbIkN

$\hookrightarrow$ vbXBpbGVyI109ImludGVsIix9LG1UPXtjaHBjPXtbImZuIl0

$\hookrightarrow$ 9Ii91dWZzL2NocGMudXRhaC51ZHUvc3lzL21vZHVsZWZpbGV 」

$\hookrightarrow \quad$ zLONIUEMtMTgVQ29yZS9jaHBjLzEuMC5sdWEiLFsiZnVsbE5」

$\hookrightarrow$ hbWUiXT0iY2hwYy8xLjAiLFsibG9hZE9yZGVyIl09MSxwcm9 」

$\hookrightarrow \quad$ wVD17fSxbInN0YWNrRGVwdGgiXTOWLFsic3RhdHVzI109ImF ।

$\hookrightarrow$ jdG12ZSIsWyJ1c2VyTmFtZSJdPSJjaHBjIix9LGludGVsPXt

$\hookrightarrow$ bImZuI109Ii91dWZzL2NocGMudXRhaC51ZHUvc3lzL21vZHV 」

$\hookrightarrow$ sZWZpbGVZLONIUEMtMTgVQ29yZS9pbnRIbC8yMDE5LjIu

MAIL=/var/spool/mail/USER

$\mathrm{PATH}=/ \mathrm{uufs} / \mathrm{chpc}$. utah. edu/sys/installdir/gcc/8.1.0/bi $\hookrightarrow \mathrm{n}: /$ uufs/chpc.utah.edu/sys/installdir/intel/compi 」

$\hookrightarrow$ lers_and_libraries_2019.2.187/linux/bin/intel64:」

$\hookrightarrow$ /uufs/chpc.utah.edu/sys/installdir/intel/compile 」

$\hookrightarrow$ rs_and_libraries_2019.2.187/linux/mpi/intel64/li 」

$\hookrightarrow$ bfabric/bin:/uufs/chpc.utah.edu/sys/installdir/i 」

$\hookrightarrow$ ntel/compilers_and_libraries_2019.2.187/linux/mp 」

$\hookrightarrow \quad$ i/intel64/bin:/uufs/chpc.utah.edu/sys/installdir 」

$\hookrightarrow$ /intel/debugger_2019/gdb/intel64/bin:/uufs/chpc. 」

$\hookrightarrow$ utah.edu/common/home/USER/brickv2/env/bin:/usr/l」

$\hookrightarrow$ ib64/qt-3.3/bin:/usr/kerberos/sbin:/usr/kerberos」

$\hookrightarrow$ /bin:/usr/lib64/ccache:/usr/local/bin:/usr/bin:/」

$\hookrightarrow$ usr/local/sbin:/usr/sbin:/uufs/notchpeak. peaks/s」

$\hookrightarrow$ ys/installdir/slurm/std/bin:/opt/dell/srvadmin/b」

$\hookrightarrow$ in:/uufs/chpc.utah.edu/common/home/USER/bin

SLURM_TASKS_PER_NODE $=1$
SLURM_WORKING_CLUSTER=notchpeak: 10.242.67.21:6817:81」

$\hookrightarrow \quad 92$

_ $=/$ usr $/ \mathrm{bin} / \mathrm{env}$

SLURM_JOB_ID $=289502$

TBBROOT=/uufs/chpc . utah. edu/sys/installdir/intel/com 」

$\hookrightarrow$ pilers_and_libraries_2019.2.187/linux/tbb

$\mathrm{PWD}=/ \mathrm{uufs} / \mathrm{chpc}$. utah . edu/common/home/USER/brickv2/Aut 」

$\hookrightarrow$ hor-Kit

SLURM_JOB_USER=USER

_LMFILES_=/uufs/chpc.utah.edu/sys/modulefiles/CHPC- 1 」

$\hookrightarrow$ 8/Core/chpc/1.0.lua:/uufs/chpc.utah.edu/sys/modu」

$\hookrightarrow$ lefiles/CHPC-18/Core/intel/2019.2.187.lua

LANG=en_US.UTF-8

MODULEPATH=/uufs/chpc. utah. edu/sys/modulefiles/CHPC- 」

$\hookrightarrow$ 18/Compiler/intel/2019.2.187:/uufs/chpc.utah.edu J

$\hookrightarrow$ /sys/modulefiles/CHPC-18/Linux:/uufs/chpc.utah.e

$\hookrightarrow$ du/sys/modulefiles/CHPC-18/Core:/uufs/chpc.utah.

$\hookrightarrow$ edu/sys/installdir/lmod/7.7.29/modulefiles/Core

KDEDIRS $=/$ usr

ModuleTable_Sz_=3

LOADEDMODULES $=$ chpc $/ 1.0:$ intel $/ 2019.2 .187$

SLURM_JOB_UID $=1080628$

SLURM_NODEID $=0$

SLURM_SUBMIT_DIR=/uufs/chpc. utah.edu/common/home/USE 」

$\hookrightarrow$ R/brickv2/Author-Kit

LMOD_CMD=/uufs/chpc. utah.edu/sys/installdir/lmod/7.7 」 $\hookrightarrow \quad .29 / 1 \mathrm{ibexec} / 1 \mathrm{mod}$

SLURM_NPROCS $=1$

SLURM_TASK_PID $=385164$

SLURM_CPUS_ON_NODE $=32$

DAALROOT=/uufs/chpc . utah. edu/sys/installdir/intel/co 」

$\hookrightarrow$ mpilers_and_libraries_2019.2.187/linux/daal

SSH_ASKPASS=/usr/libexec/openssh/gnome-ssh-askpass

HISTCONTROL=ignoredups

KRB5CCNAME=KEYRING : persistent : 1080628

ENVIRONMENT $=$ BATCH

SLURM_PROCID $=0$

SLURM_JOB_NODELIST $=$ notch 035

INTEL_PYTHONHOME=/uufs/chpc.utah.edu/sys/installdir/ 」 $\hookrightarrow \quad$ intel/debugger_2019/python/intel64/

SHLVL=3

HOME=/uufs/chpc. utah. edu/common/home/USER

SLURM_LOCALID $=0$ 
__LMOD_REF_COUNT_PATH=/uufs/chpc.utah.edu/sys/instal 」 $\hookrightarrow \quad$ ldir/intel/compilers_and_libraries_2019.2.187/li 」 $\hookrightarrow$ nux/bin/intel64:1;/uufs/chpc.utah.edu/sys/instal」 $\hookrightarrow \quad$ ldir/intel/compilers_and_libraries_2019.2.187/li 」 $\hookrightarrow$ nux/mpi/intel64/libfabric/bin:1;/uufs/chpc.utah. 」 $\hookrightarrow$ edu/sys/installdir/intel/compilers_and_libraries」 $\hookrightarrow$ _2019.2.187/linux/mpi/intel64/bin:1;/uufs/chpc.u」 $\hookrightarrow$ tah.edu/sys/installdir/intel/debugger_2019/gdb/i 」 $\hookrightarrow$ ntel64/bin:1;/uufs/chpc.utah.edu/common/home/USE 」 $\hookrightarrow$ R/brickv2/env/bin:1;/usr/lib64/qt-3.3/bin:1;/usr 」 $\hookrightarrow$ /kerberos/sbin:1;/usr/kerberos/bin:1;/usr/lib64/」 $\hookrightarrow$ ccache:1;/usr/local/bin:1;/usr/bin:1;/usr/local/」 $\hookrightarrow$ sbin:1;/usr/sbin:1;/uufs/notchpeak.peaks/sys/ins」 $\hookrightarrow$ talldir/slurm/std/bin:1;/opt/dell/srvadmin/bin:1」 $\hookrightarrow \quad$;/uufs/chpc.utah.edu/common/home/USER/bin:1

_ModuleTable002_=MTg3Lmx1YSIsWyJmdWxsTmFtZSJdPSJpbnR 」

$\hookrightarrow \quad$ lbc8yMDE5LjIUMTg3IixbImxvYWRPcmRlciJdPTIscHJvcFQ 」

$\hookrightarrow 9 e 30$ sWyJzdGFja0RlcHRoIl09MCXbInN0YXR1cyJdPSJhY3R」

$\hookrightarrow$ pdmUiLFsidXNlck5hbWUiXT0iaW50ZWwVMjAxOS4yLjE4NyI

$\hookrightarrow$ sfSX9LG1wYXRoQT17Ii91dWZzL2NocGMudXRhaC51ZHUvc31」

$\hookrightarrow \quad$ zL21vZHVsZWZpbGVZLONIUEMtMTgVQ29tcGlsZXIvaW50ZWw

$\hookrightarrow \quad$ VMjAxOS4yLjE4NyIsIi91dWZzL2NocGMudXRhaC51ZHUvc31 」

$\hookrightarrow \quad$ zL21vZHVsZWZpbGVZLONIUEMtMTgVTGludXgiLCIvdXVmcy9

$\hookrightarrow \quad$ jaHBjLnV0YWguZWR1L3N5cy9tb2R1bGVmaWxlcy9DSFBDLTE 」

$\hookrightarrow \quad 4 L 0 N v c m U i L C I v d X V m c y 9 j a H B j L n V 0 Y W g u Z W R 1 L 3 N 5 c y 9 p b n N_{J}$

$\hookrightarrow$ OYWxsZGlyL2xtb2QvNy43LjI5L21vZHVsZWZpbGVzLONv

SLURM_CLUSTER_NAME=notchpeak

SLURM_JOB_CPUS_PER_NODE $=32$

SLURM_JOB_GID $=2030$

SLURM_SUBMIT_HOST=notchpeak1

SLURM_GTIDS $=0$

BASH_ENV=/uufs/chpc.utah.edu/sys/installdir/lmod/7.7」 $\hookrightarrow .29 /$ init/bash

SLURM_JOB_PARTITION=notchpeak - freecycle

MODULERCFILE=/uufs/chpc. utah. edu/sys/modulefiles/etc 」

$\hookrightarrow \quad / \mathrm{rC}$

LOGNAME $=$ USER

QTLIB=/usr/lib64/qt-3.3/lib

CVS_RSH=ssh

BASHRC_LOADED $=1$

XDG_DATA_DIRS=/uufs/chpc. utah. edu/common/home/USER/. ।

$\hookrightarrow$ local/share/flatpak/exports/share:/var/lib/flatp」

$\hookrightarrow$ ak/exports/share:/usr/local/share:/usr/share

SSH_CONNECTION=155.97.232.235 53200 155.101.26.78 22 SLURM_JOB_ACCOUNT=ACCT

CLASSPATH=/uufs/chpc. utah. edu/sys/installdir/intel// ।

$\hookrightarrow$ compilers_and_libraries_2019.2.187/linux/mpi/int 」

$\hookrightarrow$ el64/lib/mpi.jar:/uufs/chpc.utah.edu/sys/install」

$\hookrightarrow$ dir/intel/compilers_and_libraries_2019.2.187/lin」

$\hookrightarrow \mathrm{ux} / \mathrm{daal} / \mathrm{lib} / \mathrm{daal}$.jar

KMP_HW_SUBSET $=1 \mathrm{~s}$

MODULESHOME=/uufs/chpc . utah. edu/sys/installdir/lmod/ 」

$\hookrightarrow \quad 7.7 .29$

SLURM_JOB_NUM_NODES $=1$

LESSOPEN=||/usr/bin/lesspipe. sh \%s

LMOD_SETTARG_FULL_SUPPORT=no
PKG_CONFIG_PATH=/uufs/chpc.utah.edu/sys/installdir/i 」

$\hookrightarrow$ ntel/compilers_and_libraries_2019.2.187/linux/mk」

$\hookrightarrow \quad 1 /$ bin/pkgconfig

OMP_PLACES=cores

_Init_Default_Modules=1

INTELROOT=/uufs/chpc. utah.edu/sys/installdir/intel/c 」

$\hookrightarrow$ ompilers_and_libraries_2019.2.187/linux

arch=intel64

INFOPATH=/uufs/chpc . utah. edu/sys/installdir/intel/do 」

$\hookrightarrow$ cumentation_2019/en/debugger/gdb-ia/info/

LMOD_FAMILY_COMPILER=intel

XDG_RUNTIME_DIR=/run/user $/ 1080628$

QT_PLUGIN_PATH=/usr/lib64/kde4/plugins: /usr/lib/kde4 」

$\hookrightarrow$ /plugins

OSREL=CentOS

LMOD_DIR=/uufs/chpc. utah.edu/sys/installdir/lmod/7.7 」

$\hookrightarrow .29 / 1$ ibexec

__LMOD_REF_COUNT_MANPATH=/uufs/chpc.utah.edu/sys/ins 」

$\hookrightarrow$ talldir/intel/documentation_2019/en/man/common:1 」

$\hookrightarrow \quad ; /$ uufs/chpc.utah.edu/sys/installdir/intel/compil」

$\hookrightarrow$ ers_and_libraries_2019.2.187/linux/mpi/man:1;/uu 」

$\hookrightarrow$ fs/chpc.utah.edu/sys/installdir/intel/documentat

$\hookrightarrow$ ion_2019/en/debugger/gdb-ia/man: 1 ;/uufs/chpc.uta

$\hookrightarrow$ h.edu/sys/installdir/lmod/7.7.29/share/man:1;/us」

$\hookrightarrow \mathrm{r} /$ kerberos/man:1;/usr/local/share/man: 1 ;/usr/sha

$\hookrightarrow \mathrm{re} / \mathrm{man} /$ overrides:1;/usr/share/man:1;/uufs/notchp

$\hookrightarrow$ eak.peaks/sys/installdir/slurm/std/share/man:2

SLURM_MEM_PER_NODE $=102400$

I_MPI_ROOT=/uufs/chpc.utah.edu/sys/installdir/intel/ 」

$\hookrightarrow$ /compilers_and_libraries_2019.2.187/linux/mpi

BASH_FUNC_module ()$=()\left\{\right.$ eval $\$\left(\$ L M O D \_C M D\right.$ bash "\$Q")

$\hookrightarrow \quad$ \&\& eval $\$\left(\$\left\{L M O D \_S E T T A R G \_C M D:-:\right\}-s \quad s h\right)$

\}

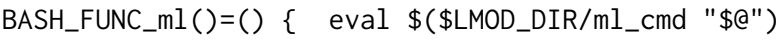

\}

+ 1sb_release -a

LSB Version: :core-4.1-amd64:core-4.1-noarch:

$\hookrightarrow$ cxx-4.1-amd64:cxx-4.1-noarch:desktop-4.1-amd64:d J

$\hookrightarrow$ esktop-4.1-noarch: languages-4.1-amd64: languages-

$\hookrightarrow$ 4.1-noarch:printing-4.1-amd64:printing-4.1-noarch Distributor ID: CentOS

Description: CentOS Linux release 7.6.1810

$\hookrightarrow$ (Core)

Release: $\quad$ 7.6.1810

Codename: Core

+ uname -a

Linux notch035 3.10.0-957.1.3.el7.x86_64 \#1 SMP Thu

$\hookrightarrow$ Nov 29 14:49:43 UTC 2018 x86_64 x86_64 x86_64

$\hookrightarrow \quad$ GNU/Linux

+ lscpu

Architecture:

x86_64

CPU op-mode(s): $\quad$ 32-bit, 64-bit

Byte Order:

$\mathrm{CPU}(\mathrm{s})$ :

Little Endian

On-line CPU(s) list: $0-63$

Thread(s) per core: 2 
Exploiting Reuse and Vectorization in Blocked Stencil Computations on CPUs and GPUs




+ eval 'MODULEPATH="/uufs/chpc.utah.edu/sys/modulefi

$\hookrightarrow$ les/CHPC-18/Compiler/intel/2019.2.187:/uufs/chpc」

$\hookrightarrow$.utah.edu/sys/modulefiles/CHPC-18/Linux:/uufs/ch」

$\hookrightarrow$ pc.utah.edu/sys/modulefiles/CHPC-18/Core:/uufs/c 」

$\hookrightarrow$ hpc.utah.edu/sys/installdir/lmod/7.7.29/modulefi

$\hookrightarrow$ les/Core"; '

export 'MODULEPATH;' '_ModuleTable001_="X01vZHVsZVRh」

$\hookrightarrow \quad Y m x 1 X z 17$ WyJNVHZlcnNpb24iXT0zLFsiY19yZWJ1aWxkVGlt

$\hookrightarrow$ ZSJdPWZhbHN1LFsiY19zaG9ydFRpbWUiXT1mYWxzZSxkZXB0,

$\hookrightarrow \quad$ aFQ9e30sZmFtaWx5PXtbIkNvbXBpbGVyI109ImludGVsIix9 」

$\hookrightarrow$ LG1UPXtjaHBjPXtbImZuI109Ii91dWZzL2NocGMudXRhaC51」

$\hookrightarrow \quad$ ZHUvc3lzL21vZHVsZWZpbGVZLONIUEMtMTgvQ29yZS9jaHBj」

$\hookrightarrow$ LzEuMC5sdWEiLFsiZnVsbE5hbWUiXT0iY2hwYy8xLjAiLFsi

$\hookrightarrow$ bG9hZE9yZGVyI109MSxwcm9wVD17fSxbInNOYWNrRGVwdGgi ।

$\hookrightarrow \quad$ XT0wLFsic3RhdHVzI109ImFjdGl2ZSIsWyJ1c2VyTmFtZSJd

$\hookrightarrow$ PSJjaHBjIix9LGludGVsPXtbImZuI109Ii91dWZzL2NocGMu 」

$\hookrightarrow$ dXRhaC51ZHUvc3lzL21vZHVsZWZpbGVzLONIUEMtMTgVQ29y

$\hookrightarrow \quad$ ZS9pbnRlbC8yMDE5LjIu"; '

export '_ModuleTable001_;' '_ModuleTable002_="MTg3Lm」

$\hookrightarrow \quad x 1 Y S I s W y J m d W x s T m F t Z S J d P S J p b n R I b C 8 y M D E 5 L j I$ IUMTg3Ii

$\hookrightarrow \quad x b I m x v Y W R P c m R l c i J d P T I s c H J v c F Q 9 e 30 s W y J z d G F j a 0 R l c H$ 」

$\hookrightarrow$ RoI109MCXbInNOYXR1cyJdPSJhY3RpdmUiLFsidXNlck5hbW

$\hookrightarrow \quad$ UiXT0iaW50ZWwVMjAxOS4yLjE4NyIsfSx9LG1wYXRoQT17Ii 」

$\hookrightarrow \quad 91 \mathrm{dWZzL} 2$ NocGMudXRhaC51ZHUvc3lzL21vZHVsZWZpbGVzL0 」

$\hookrightarrow \quad$ NIUEMtMTgvQ29tcGlsZXIvaW50ZWwvMjAxOS4yLjE4NyIsIi 」

$\hookrightarrow \quad 91 \mathrm{dWZzL} 2$ NocGMudXRhaC51ZHUvc3lzL21vZHVsZWZpbGVzL0 」

$\hookrightarrow \quad$ NIUEMtMTgvTGludXgiLCIvdXVmcy9jaHBjLnVOYWguZWR1L3 」

$\hookrightarrow \quad$ N5cy9tb2R1bGVmaWx lcy9DSFBDLTE4LONvcmUiLCIvdXVmcy 」

$\hookrightarrow \quad$ 9jaHBjLnVOYWguZWR1L3N5cy9pbnNOYWxsZGlyL2xtb2QvNy 」

$\hookrightarrow$ 43LjI5L21vZHVsZWZpbGVZLONv"; '

export '_ModuleTable002_;'

$\hookrightarrow$ '_ModuleTable003_="cmUiLH0sWyJzeXNOZW1CYXNITVBBV」

$\hookrightarrow$ EgiXT0iL3V1ZnMvY2hwYy51dGFoLmVkdS9zeXMvbW9kdWxlZ 」

$\hookrightarrow \quad$ mlsZXMvQ0hQQy0xOC9MaW51eDovdXVmcy9jaHBjLnV0YWguZ」

$\hookrightarrow \quad$ WR1L3N5cy9tb2R1bGVmaWxlcy9DSFBDLTE4LONvcmU6L3V1Z 」

$\hookrightarrow$ nMvY2hwYy51dGFoLmVkdS9zeXMvaW5zdGFsbGRpci9sbW9kL 」

$\hookrightarrow \quad$ zcuNy4y0S9tb2R1bGVmaWxlcy9Db3JlIix9"; '

export '_ModuleTable003_; ' 'ModuleTable_Sz_="3";

export '_ModuleTable_Sz_;'

++ MODULEPATH=/uufs/chpc.utah.edu/sys/modulefiles/CH」

$\hookrightarrow$ PC-18/Compiler/intel/2019.2.187:/uufs/chpc.utah. 」

$\hookrightarrow$ edu/sys/modulefiles/CHPC-18/Linux:/uufs/chpc.uta J

$\hookrightarrow$ h.edu/sys/modulefiles/CHPC-18/Core:/uufs/chpc.ut

$\hookrightarrow$ ah.edu/sys/installdir/lmod/7.7.29/modulefiles/Co」

$\hookrightarrow$ re

$++$

export MODULEPATH
++_ModuleTable001_=X01 vZHVsZZVRhYmxIXz17WyJNVHZlcnNp 」

$\hookrightarrow$ b24iXT0ZLFsiY19yZWJ1aWxkVGltZSJdPWZhbHN1LFsiY19z 」

$\hookrightarrow \quad$ aG9ydFRpbWUiXT1mYWxzZSxkZXB0aFQ9e30sZmFtaWx5PXtb」

$\hookrightarrow \quad$ IkNvbXBpbGVyI109ImludGVsIix9LG1UPXtjaHBjPXtbImZu」

$\hookrightarrow \quad$ Il09Ii91dWZzL2NocGMudXRhaC51ZHUvc3lzL21vZHVsZWZp 」

$\hookrightarrow \quad$ bGVZLONIUEMtMTgVQ29yZS9jaHBjLzEuMC5sdWEiLFsiZnVs 」

$\hookrightarrow \quad$ bE5hbWUiXT0iY2hwYy8xLjAiLFsibG9hZE9yZGVyIl09MSxw

$\hookrightarrow \quad \mathrm{cm} 9 w V D 17 f S x b I n N 0 Y W N r R G V w d G g i X T 0 w L F s i c 3 R h d H V z I 109$ 」

$\hookrightarrow \quad$ ImFjdGl2ZSIsWyJ1c2VyTmFtZSJdPSJjaHBjIix9LGludGVs 」

$\hookrightarrow$ PXtbImZuI109Ii91dWZzL2NocGMudXRhaC51ZHUvc3lzL21v」

$\hookrightarrow \quad$ ZHVsZWZpbGVzLONIUEMtMTgVQ29yZS9pbnRIbC8yMDE5LjIu

$++$

export_ModuleTable001_

++ _ModuleTable002_=MTg3Lmx1YSIsWyJmdWxsTmFtZSJdPSJp 」

$\hookrightarrow$ bnRIbC8yMDE5LjIuMTg3I i xbImxvYWRPcmRIciJdPTIscHJv 」

$\hookrightarrow \quad$ cFQ9e30sWyJzdGFja0RlcHRoI109MCxbInNOYXR1cyJdPSJh

$\hookrightarrow \quad$ Y3RpdmUiLFsidXNlck5hbWUiXT0iaW50ZWwvMjAxOS4yLjE4」

$\hookrightarrow$ NyIsfSx9LG1wYXRoQT17Ii91dWZzL2NocGMudXRhaC51ZHUv 」

$\hookrightarrow$ c3lzL21VZHVsZWZpbGVZL0NIUEMtMTgVQ29tcGlsZXIvaW50 」

$\hookrightarrow \quad$ ZWwvMjAxOS4yLjE4NyIsIi91dWZzL2NocGMudXRhaC51ZHUv 」

$\hookrightarrow \quad c 31 z L 21 v Z H V s Z W Z p b G V z L 0 N I U E M t M T g v T G l u d X g i L C I v d X V m_{」}$

$\hookrightarrow \quad$ cy9jaHBjLnV0YWguZWR1L3N5cy9tb2R1bGVmaWxlcy9DSFBD 」

$\hookrightarrow \quad$ LTE4LONvcmUiLCIvdXVmcy9jaHBjLnV0YWguZWR1L3N5cy9p 」

$\hookrightarrow \quad$ bnNOYWxsZGlyL2xtb2QvNy43LjI5L21vZHVsZWZpbGVzLONv

$++$

export_ModuleTable002_

++_ModuleTable003_=cmUiLH0sWyJzeXN0ZW1CYXN1TVBBVEgi 」

$\hookrightarrow \quad X T 0 i L 3 V 1 Z n M v Y 2 h w Y y 51 d G F o L m V k d S 9 z e X M v b W 9 k d W x 1 Z m l s$ 」

$\hookrightarrow \quad$ ZXMvQ0hQQy0xOC9MaW51eDovdXVmcy9jaHBjLnV0YWguZWR1」

$\hookrightarrow \quad$ L3N5cy9tb2R1bGVmaWxlcy9DSFBDLTE4LONvcmU6L3V1ZnMv 」

$\hookrightarrow \quad$ Y2hwYy51dGFoLmVkdS9zeXMvaW5zdGFsbGRpci9sbW9kLzcu」

$\hookrightarrow$ Ny4y0S9tb2R1bGVmaWxlcy9Db3J1Iix9

$++$

export_ModuleTable003_

++ _ModuleTable_Sz_=3

$++$

export_ModuleTable_Sz_

++ : $-\mathrm{s}$ sh

+ eval

+ nvidia-smi

NVIDIA-SMI has failed because it couldn't communicate

$\hookrightarrow$ with the NVIDIA driver. Make sure that the latest

$\hookrightarrow$ NVIDIA driver is installed and running.

+ lshw -short -quiet -sanitize

+ cat

WARNING: you should run this program as super-user.

$\mathrm{H} / \mathrm{W}$ path Device Class Description

\begin{tabular}{|c|c|c|}
\hline & system & Computer \\
\hline 10 & bus & Motherboard \\
\hline $10 / 1$ & memory & 190GiB System \\
\hline \multicolumn{3}{|c|}{$\hookrightarrow \quad$ memory } \\
\hline $10 / 3$ & processor & Intel(R) \\
\hline
\end{tabular}


Exploiting Reuse and Vectorization in Blocked Stencil Computations on CPUs and GPUs

\begin{tabular}{|c|c|c|c|c|}
\hline processor & Intel (R) & $10 / 2$ & bridge & Sky Lake-E PCI \\
\hline $\begin{array}{c}\hookrightarrow \quad \operatorname{Xeon}(\mathrm{R}) \text { Gold } 6130 \mathrm{CPU} \text { @ } 2.10 \mathrm{GHz} \\
10 / 100 \quad \text { bridge }\end{array}$ & Sky Lake-E DMI3 & $\begin{array}{l}\hookrightarrow \text { Express Root } \\
/ 0 / 2 / 0\end{array}$ & storage & MegaRAID SAS-3 \\
\hline$\hookrightarrow$ Registers & & $\hookrightarrow \quad 3008$ [Fury] & & \\
\hline$/ 0 / 100 / 5$ & Sky Lake-E & $10 / 6$ & generic & Intel Corporation \\
\hline$\hookrightarrow \quad M M / V t-d$ Configuration Registers & & $10 / 7$ & generic & Sky Lake-E RAS \\
\hline $10 / 100 / 5.2$ & Intel & $\hookrightarrow$ Configuration & sters & \\
\hline$\hookrightarrow$ Corporation & & $10 / 9$ & generic & Intel Corporation \\
\hline $10 / 100 / 5.4$ & Intel & $10 / a$ & generic & Sky Lake-E CHA \\
\hline$\hookrightarrow$ Corporation & & $\hookrightarrow$ Registers & & \\
\hline $\begin{array}{l}10 / 100 / 8 \\
\hookrightarrow \quad \text { Registers }\end{array}$ & Sky Lake-E Ubox & $\begin{array}{l}10 / \mathrm{b} \\
\hookrightarrow \text { Registers }\end{array}$ & generic & Sky Lake-E CHA \\
\hline $\begin{array}{l}10 / 100 / 8.1 \\
\hookrightarrow \quad \text { Registers }\end{array}$ & Sky Lake-E Ubox & $\begin{array}{l}10 / c \\
\hookrightarrow \quad \text { Registers }\end{array}$ & generic & Sky Lake-E CHA \\
\hline $\begin{array}{ll}10 / 100 / 8.2 & \text { generic } \\
\hookrightarrow \quad \text { Registers } & \end{array}$ & Sky Lake-E Ubox & $\begin{array}{l}/ 0 / d \\
\hookrightarrow \text { Registers }\end{array}$ & generic & Sky Lake-E CHA \\
\hline$/ 0 / 100 / 11$ & C620 Series & $10 / \mathrm{e}$ & generic & Sky Lake-E CHA \\
\hline $\begin{array}{l}\hookrightarrow \text { Chipset Family MROM } 0 \\
/ 0 / 100 / 11.5\end{array}$ & C620 Series & $\begin{array}{l}\hookrightarrow \text { Registers } \\
/ 0 / f\end{array}$ & generic & Sky Lake-E CHA \\
\hline $\begin{array}{l}\hookrightarrow \text { Chipset Family SSATA Controller [AF } \\
10 / 100 / 14 \\
\text { bus }\end{array}$ & $\begin{array}{l}\text { HCI mode] } \\
\text { C620 Series }\end{array}$ & $\begin{array}{l}\hookrightarrow \quad \text { Registers } \\
/ 0 / 10\end{array}$ & generic & Sky Lake-E CHA \\
\hline$\hookrightarrow$ Chipset Family USB 3.0 xHCI Contro] & ller & $\hookrightarrow$ Registers & & \\
\hline $\begin{array}{l}\text { /0/100/14.2 } \\
\hookrightarrow \quad \text { Chipset Family Thermal Subsystem }\end{array}$ & C620 Series & $\begin{array}{l}\text { /0/11 } \\
\hookrightarrow \quad \text { Registers }\end{array}$ & generic & Sky Lake-E CHA \\
\hline $10 / 100 / 16 \quad$ communication & C620 Series & $10 / 12$ & generic & Sky Lake-E CHA \\
\hline $\begin{array}{l}\hookrightarrow \quad \text { Chipset Family MEI Controller \#1 } \\
/ 0 / 100 / 16.1\end{array}$ & C620 Series & $\begin{array}{l}\hookrightarrow \text { Registers } \\
/ 0 / 13\end{array}$ & generic & Sky Lake-E CHA \\
\hline $\begin{array}{l}\hookrightarrow \quad \text { Chipset Family MEI Controller \#2 } \\
/ 0 / 100 / 16.4 \\
\text { communication }\end{array}$ & C620 Series & $\begin{array}{l}\hookrightarrow \text { Registers } \\
/ 0 / 14\end{array}$ & generic & Sky Lake-E CHA \\
\hline $\begin{array}{l}\hookrightarrow \quad \text { Chipset Family MEI Controller \#3 } \\
/ 0 / 100 / 17\end{array}$ & C620 Series & $\begin{array}{l}\hookrightarrow \text { Registers } \\
/ 0 / 15\end{array}$ & generic & Sky Lake-E CHA \\
\hline $\begin{array}{l}\hookrightarrow \quad \text { Chipset Family SATA Controller [AHC } \\
10 / 100 / 1 \mathrm{c} \\
\text { bridge }\end{array}$ & $\begin{array}{l}\text { CI mode] } \\
\text { C620 Series }\end{array}$ & $\begin{array}{l}\hookrightarrow \quad \text { Registers } \\
/ 0 / 16\end{array}$ & generic & Sky Lake-E CHA \\
\hline$\hookrightarrow$ Chipset Family PCI Express Root Por & t \#1 & $\hookrightarrow$ Registers & & \\
\hline 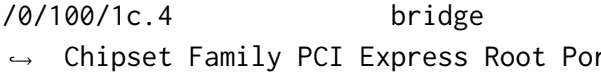 & $\begin{array}{l}\text { C620 Series } \\
\text { t \#5 }\end{array}$ & $\begin{array}{l}\text { /0/17 } \\
\hookrightarrow \quad \text { Registers }\end{array}$ & generic & Sky Lake-E CHA \\
\hline $\begin{array}{ll}/ 0 / 100 / 1 c \cdot 4 / 0 & \text { bridge } \\
/ 0 / 100 / 1 c \cdot 4 / 0 / 0 & \text { display }\end{array}$ & $\begin{array}{l}\text { PLDA } \\
\text { Integrated }\end{array}$ & $\begin{array}{l}\text { /0/18 } \\
\hookrightarrow \quad \text { Registers }\end{array}$ & generic & Sky Lake-E CHA \\
\hline $\begin{array}{l}\hookrightarrow \text { Matrox G200eW3 Graphics Controller } \\
10 / 100 / 1 \text { c. } 5 \\
\text { bridge }\end{array}$ & C620 Series & $\begin{array}{l}/ 0 / 19 \\
\hookrightarrow \quad \text { Registers }\end{array}$ & generic & Sky Lake-E CHA \\
\hline $\begin{array}{l}\hookrightarrow \text { Chipset Family PCI Express Root Por } \\
10 / 100 / 1 \mathrm{c} .5 / 0 \quad \text { eth } 0 \text { network }\end{array}$ & $\begin{array}{l}\text { NetXtreme } \\
\text { Net }\end{array}$ & $\begin{array}{l}/ 0 / 1 a \\
\hookrightarrow \quad \text { Registers }\end{array}$ & generic & Sky Lake-E CHA \\
\hline $\begin{array}{l}\hookrightarrow \quad \text { BCM5720 Gigabit Ethernet PCIe } \\
10 / 100 / 1 \text { c.5/0.1 eth1 network }\end{array}$ & NetXtreme & $\begin{array}{l}10 / 1 \mathrm{~b} \\
\hookrightarrow \quad \text { Registers }\end{array}$ & generic & Sky Lake-E CHA \\
\hline $\begin{array}{l}\hookrightarrow \quad \text { BCM5720 Gigabit Ethernet PCIe } \\
10 / 100 / 1 \mathrm{f} \quad \text { bridge }\end{array}$ & C621 Series & $\begin{array}{l}10 / 1 \mathrm{c} \\
\hookrightarrow \quad \text { Registers }\end{array}$ & generic & Sky Lake-E CHA \\
\hline$\hookrightarrow \quad$ Chipset LPC/eSPI Controller & & $/ 0 / 1 d$ & generic & Sky Lake-E CHA \\
\hline $\begin{array}{ll}10 / 100 / 1 \mathrm{f} .2 & \text { memory } \\
\hookrightarrow \quad \text { controller } & \end{array}$ & Memory & $\begin{array}{l}\hookrightarrow \text { Registers } \\
/ 0 / 1 \mathrm{e}\end{array}$ & generic & Sky Lake-E CHA \\
\hline $10 / 100 / 1 \mathrm{f} .4$ bus & C620 Series & $\begin{array}{l}\hookrightarrow \quad \text { Registers } \\
/ 0 / 1 \mathrm{f}\end{array}$ & & \\
\hline $\begin{array}{l}\hookrightarrow \quad \text { Chipset Family SMBus } \\
10 / 100 / 1 \mathrm{f} .5 \quad \text { bus }\end{array}$ & C620 Series & $\begin{array}{l}\text { 10/1f } \\
\hookrightarrow \quad \text { Registers }\end{array}$ & generic & Sky Lake-E CHA \\
\hline$\hookrightarrow$ Chipset Family SPI Controller & & $\begin{array}{l}/ 0 / 20 \\
\hookrightarrow \quad \text { Registers }\end{array}$ & generic & Sky Lake-E CHA \\
\hline
\end{tabular}




\begin{tabular}{|c|c|c|c|c|c|}
\hline $10 / 21$ & generic & Sky Lake-E CHA & $10 / 3 c$ & generic & Sky Lake-E CHA \\
\hline$\hookrightarrow$ Registers & & & $\hookrightarrow$ Registers & & \\
\hline $10 / 22$ & generic & Sky Lake-E CHA & $10 / 3 d$ & generic & Sky Lake-E CHA \\
\hline$\hookrightarrow$ Registers & & & $\hookrightarrow$ Registers & & \\
\hline $10 / 23$ & generic & Sky Lake-E CHA & $10 / 3 \mathrm{e}$ & generic & Sky Lake-E CHA \\
\hline$\hookrightarrow$ Registers & & & $\hookrightarrow$ Registers & & \\
\hline $10 / 24$ & generic & Sky Lake-E CHA & $10 / 3 f$ & generic & Sky Lake-E CHA \\
\hline$\hookrightarrow$ Registers & & & $\hookrightarrow$ Registers & & \\
\hline $10 / 25$ & generic & Sky Lake-E CHA & $10 / 40$ & generic & Sky Lake-E CHA \\
\hline$\hookrightarrow$ Registers & & & $\hookrightarrow$ Registers & & \\
\hline $10 / 26$ & generic & Sky Lake-E CHA & $10 / 41$ & generic & Sky Lake-E CHA \\
\hline$\hookrightarrow$ Registers & & & $\hookrightarrow$ Registers & & \\
\hline $10 / 27$ & generic & Sky Lake-E CHA & $10 / 42$ & generic & Sky Lake-E CHA \\
\hline$\hookrightarrow$ Registers & & & $\hookrightarrow$ Registers & & \\
\hline $10 / 28$ & generic & Sky Lake-E CHA & $10 / 43$ & generic & Sky Lake-E CHA \\
\hline$\hookrightarrow$ Registers & & & $\hookrightarrow$ Registers & & \\
\hline $10 / 29$ & generic & Sky Lake-E CHA & $10 / 44$ & generic & Sky Lake-E CHA \\
\hline$\hookrightarrow$ Registers & & & $\hookrightarrow$ Registers & & \\
\hline $10 / 2 a$ & generic & Sky Lake-E CHA & $10 / 45$ & generic & Sky Lake-E CHA \\
\hline$\hookrightarrow$ Registers & & & $\hookrightarrow$ Registers & & \\
\hline $10 / 2 b$ & generic & Sky Lake-E CHA & $10 / 46$ & generic & Sky Lake-E PCU \\
\hline$\hookrightarrow$ Registers & & & $\hookrightarrow$ Registers & & \\
\hline $10 / 2 c$ & generic & Sky Lake-E CHA & $10 / 47$ & generic & Sky Lake-E PCU \\
\hline$\hookrightarrow$ Registers & & & $\hookrightarrow$ Registers & & \\
\hline $10 / 2 d$ & generic & Sky Lake-E CHA & $10 / 48$ & generic & Sky Lake-E PCU \\
\hline$\hookrightarrow$ Registers & & & $\hookrightarrow$ Registers & & \\
\hline $10 / 2 \mathrm{e}$ & generic & Sky Lake-E CHA & $10 / 49$ & generic & Sky Lake-E PCU \\
\hline$\hookrightarrow$ Registers & & & $\hookrightarrow$ Registers & & \\
\hline $10 / 2 f$ & generic & Sky Lake-E CHA & $10 / 4 a$ & generic & Sky Lake-E PCU \\
\hline$\hookrightarrow$ Registers & & & $\hookrightarrow$ Registers & & \\
\hline $10 / 30$ & generic & Sky Lake-E CHA & $10 / 4 b$ & generic & Sky Lake-E PCU \\
\hline$\hookrightarrow$ Registers & & & $\hookrightarrow$ Registers & & \\
\hline $10 / 31$ & generic & Sky Lake-E CHA & $10 / 4 c$ & generic & Sky Lake-E PCU \\
\hline$\hookrightarrow$ Registers & & & $\hookrightarrow$ Registers & & \\
\hline $10 / 32$ & generic & Sky Lake-E CHA & $10 / 4 d$ & generic & Intel Corporation \\
\hline$\hookrightarrow$ Registers & & & $10 / 4 \mathrm{e}$ & generic & Sky Lake-E RAS \\
\hline $10 / 33$ & generic & Sky Lake-E CHA & $\hookrightarrow$ Configura & sters & \\
\hline$\hookrightarrow$ Registers & & & $10 / 4 f$ & generic & Intel Corporation \\
\hline $10 / 34$ & generic & Sky Lake-E CHA & $10 / 50$ & generic & Intel Corporation \\
\hline$\hookrightarrow$ Registers & & & $/ 0 / 51$ & generic & Intel Corporation \\
\hline $10 / 35$ & generic & Sky Lake-E CHA & $10 / 52$ & generic & Intel Corporation \\
\hline$\hookrightarrow$ Registers & & & $10 / 53$ & generic & Intel Corporation \\
\hline $10 / 36$ & generic & Sky Lake-E CHA & $10 / 54$ & generic & Intel Corporation \\
\hline$\hookrightarrow$ Registers & & & $10 / 55$ & generic & Intel Corporation \\
\hline $10 / 37$ & generic & Sky Lake-E CHA & $10 / 56$ & generic & Intel Corporation \\
\hline$\hookrightarrow$ Registers & & & $10 / 57$ & generic & Intel Corporation \\
\hline $10 / 38$ & generic & Skv Lake-E CHA & $10 / 58$ & generic & Intel Corporation \\
\hline$\hookrightarrow$ Registers & & & $10 / 59$ & generic & Intel Corporation \\
\hline $10 / 39$ & generic & Skv I ake-F CHA & $10 / 5 a$ & generic & Intel Corporation \\
\hline$\hookrightarrow$ Registers & generic & SKy Lake-E CHA & $10 / 5 b$ & generic & Intel Corporation \\
\hline $\begin{array}{l}\hookrightarrow 0 / 3 a \\
10\end{array}$ & & & $10 / 5 c$ & generic & Intel Corporation \\
\hline 10/3a & generıc & Sky Lake-E CHA & $10 / 5 d$ & generic & Intel Corporation \\
\hline$\hookrightarrow$ Registers & & & $10 / 5 \mathrm{e}$ & generic & Intel Corporation \\
\hline $10 / 3 b$ & generic & Sky Lake-E CHA & $10 / 5 f$ & generic & Intel Corporation \\
\hline$\hookrightarrow$ Registers & & & $10 / 60$ & generic & Intel Corporation \\
\hline
\end{tabular}


Exploiting Reuse and Vectorization in Blocked Stencil Computations on CPUs and GPUs

\begin{tabular}{|c|c|c|c|c|c|}
\hline$/ 0 / 61$ & generic & Intel Corporation & $10 / 8.2$ & generic & Sky Lake-E CHA \\
\hline $10 / 62$ & generic & Intel Corporation & $\hookrightarrow$ Registers & & \\
\hline $10 / 63$ & generic & Intel Corporation & $10 / 86$ & generic & Sky Lake-E CHA \\
\hline $10 / 64$ & generic & Intel Corporation & $\hookrightarrow$ Registers & & \\
\hline $10 / 65$ & generic & Intel Corporation & $10 / 87$ & generic & Sky Lake-E CHA \\
\hline $10 / 66$ & generic & Intel Corporation & $\hookrightarrow$ Registers & & \\
\hline $10 / 67$ & generic & Intel Corporation & $10 / 88$ & generic & Sky Lake-E CHA \\
\hline $10 / 68$ & generic & Intel Corporation & $\hookrightarrow$ Registers & & \\
\hline $10 / 69$ & generic & Intel Corporation & $10 / 89$ & generic & Sky Lake-E CHA \\
\hline 10/6a & generic & Intel Corporation & $\hookrightarrow$ Registers & & \\
\hline $\begin{array}{l}\text { 10/6b } \\
\hookrightarrow \quad \text { Configuration Re }\end{array}$ & generic & Sky Lake-E RAS & $10 / 8 a$ & generic & Sky Lake-E CHA \\
\hline $\begin{array}{l}\hookrightarrow \text { Contiguration Re } \\
10 / 6 c\end{array}$ & $\begin{array}{l}\text { sters } \\
\text { generic }\end{array}$ & Intel Corporation & $\hookrightarrow$ Registers & & \\
\hline $10 / 6 \mathrm{~d}$ & generic & Intel Corporation & /0/8b & generic & Sky Lake-E CHA \\
\hline $10 / 6 \mathrm{e}$ & generic & Intel Corporation & $\hookrightarrow$ Registers & & \\
\hline $10 / 6 f$ & generic & Intel Corporation & 10/8C & generıc & Sky Lake-E CHA \\
\hline $10 / 70$ & generic & Intel Corporation & $\hookrightarrow$ Registers & & \\
\hline $10 / 71$ & generic & Intel Corporation & $/ 0 / 8 d$ & generic & Sky Lake-E CHA \\
\hline $10 / 72$ & generic & Intel Corporation & $\hookrightarrow$ Registers & & \\
\hline $10 / 73$ & generic & Sky Lake-E & $10 / 8 \mathrm{e}$ & generic & Sky Lake-E CHA \\
\hline$\hookrightarrow \quad$ M3KTI Registers & & & $\hookrightarrow$ Registers & & \\
\hline $\begin{array}{l}\text { 10/74 } \\
\quad \text { M3КTT Registers }\end{array}$ & generic & Sky Lake-E & $\begin{array}{l}10 / 8 f \\
\hookrightarrow \quad \text { Registers }\end{array}$ & generic & Sky Lake-E CHA \\
\hline $10 / 75$ & generic & Sky Lake-E & $10 / 90$ & generic & Sky Lake-E CHA \\
\hline$\hookrightarrow$ M3KTI Registers & & & $\hookrightarrow$ Registers & & \\
\hline $10 / 76$ & generic & Sky Lake-E & /0/91 & generic & Sky Lake-E CHA \\
\hline$\hookrightarrow$ M3KTI Registers & & & $\hookrightarrow$ Registers & & \\
\hline $10 / 77$ & generic & Sky Lake-E & $10 / 92$ & generic & Sky Lake-E CHA \\
\hline 10/78 & generic & Sky Lake-E & $10 / 93$ & generic & Sky Lake-E CHA \\
\hline $\begin{array}{l}\hookrightarrow \quad \text { M2PCI Registers } \\
10 / 79\end{array}$ & generic & Sky Lake-E & $\begin{array}{l}\hookrightarrow \text { Registers } \\
/ 0 / 94\end{array}$ & generic & Sky Lake-E CHA \\
\hline$\hookrightarrow \quad$ M2PCI Registers & & & $\hookrightarrow$ Registers & & \\
\hline $10 / 7 a$ & generic & Sky Lake-E & /0/95 & generic & Sky Lake-E CHA \\
\hline$\hookrightarrow \quad$ M2PCI Registers & & & $\hookrightarrow$ Registers & & \\
\hline $10 / 7 \mathrm{~b}$ & generic & Sky Lake-E & /0/96 & generic & Sky Lake-E CHA \\
\hline $\begin{array}{l}\hookrightarrow \quad \text { M2PCI Registers } \\
/ 0 / 7 \mathrm{c}\end{array}$ & & & $\hookrightarrow \quad$ Registers & rononic & Sky L \\
\hline$\hookrightarrow \quad \mathrm{MM} / \mathrm{Vt}-\mathrm{d}$ Configur & $\begin{array}{l}\text { generic } \\
\text { ion Registers }\end{array}$ & Sky Lake-E & $\hookrightarrow$ Registers & generic & Sky Lake-E CHA \\
\hline $10 / 7 d$ & generic & Intel Corporation & $10 / 98$ & generic & Sky Lake-E CHA \\
\hline $10 / 7$ e & generic & Intel Corporation & $\hookrightarrow$ Registers & & \\
\hline $10 / 7 f$ & generic & Sky Lake-E Ubox & 10/99 & generic & Sky Lake-E CHA \\
\hline$\hookrightarrow$ Registers & & & $\hookrightarrow$ Registers & & \\
\hline $10 / 80$ & generic & Sky Lake-E Ubox & $10 / 9 a$ & generic & Sky Lake-E CHA \\
\hline$\hookrightarrow$ Registers & & & $\hookrightarrow$ Registers & & \\
\hline $10 / 81$ & generic & Sky Lake-E Ubox & $10 / 9 b$ & generic & Sky Lake-E CHA \\
\hline$\hookrightarrow$ Registers & & & $\hookrightarrow$ Registers & & \\
\hline $10 / 82$ & generic & Intel Corporation & $10 / 9 c$ & generic & Sky Lake-E CHA \\
\hline $10 / 83$ & generic & Sky Lake-E RAS & $\hookrightarrow$ Registers & & \\
\hline$\hookrightarrow$ Configuration $\mathrm{Re}$ & sters & & $10 / 9 d$ & generic & Sky Lake-E CHA \\
\hline $10 / 84$ & generic & Intel Corporation & $\hookrightarrow$ Registers & & \\
\hline $10 / 85$ & generic & Sky Lake-E CHA & $10 / 9 \mathrm{e}$ & generic & Sky Lake-E CHA \\
\hline$\hookrightarrow$ Registers & & & $\hookrightarrow$ Registers & & \\
\hline 10/8.1 & generic & Sky Lake-E CHA & 10/9f & generic & Sky Lake-E CHA \\
\hline
\end{tabular}




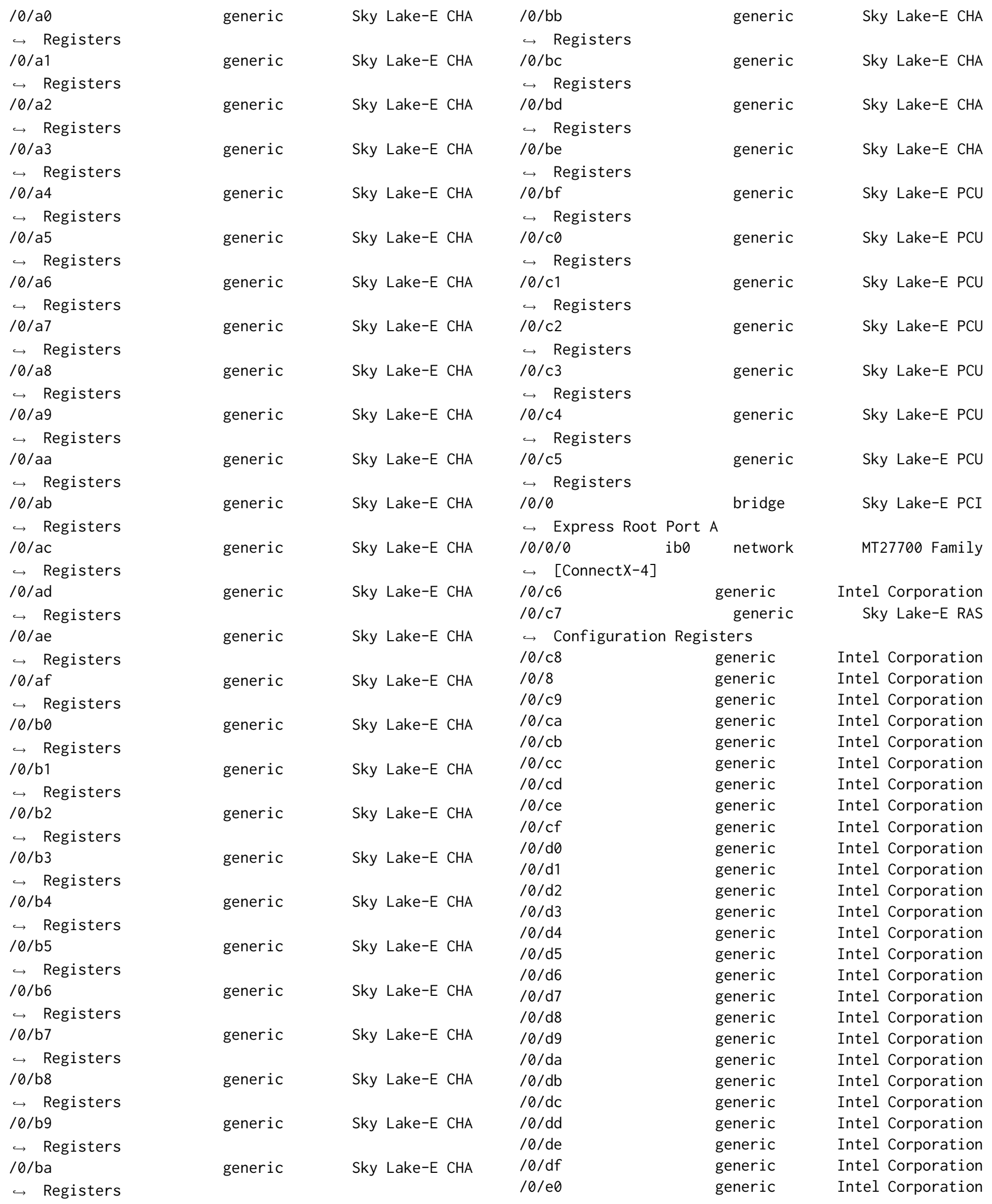


Exploiting Reuse and Vectorization in Blocked Stencil Computations on CPUs and GPUs

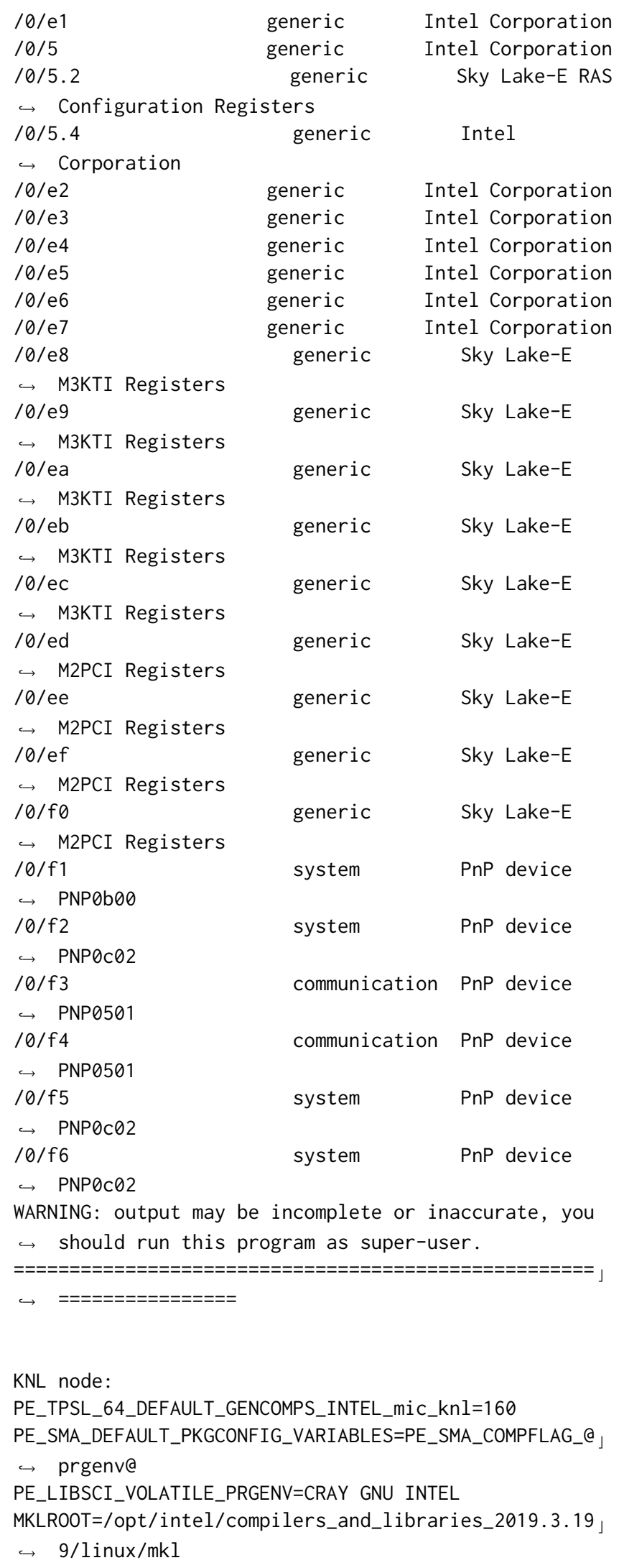

ZAP_LIBPATH=/opt/ovis/lib64/ovis-lib

KSH_AUTOLOAD $=1$

MODULE_VERSION_STACK $=3.2 .10 .6$

LESSKEY=/etc/lesskey.bin

SLURM_NODELIST=nid05865

SLURM_CHECKPOINT_IMAGE_DIR=/var/slurm/checkpoint

GNU_VERSION $=8.2 .0$

DVS_MAXNODES $=1$

PE_CXX_PKGCONFIG_LIBS=mpichcXX

PE_TPSL_DEFAULT_GENCOMPS_INTEL_X86_skylake $=160$

PE_PETSC_DEFAULT_GENCOMPS_CRAY_skylake $=86$

PE_PETSC_DEFAULT_GENCOMPILERS_CRAY_sandybridge $=8.6$

PE_PAPI_DEFAULT_ACCEL_FAMILY_LIBS_nvidia $=,-1$ cupt $i,-1$ 」

$\hookrightarrow$ cudart,-lcuda

NNTPSERVER=news

MANPATH=/usr/common/software/man:/usr/common/mss/man ।

$\hookrightarrow \quad: /$ usr/common/nsg/man:/opt/gcc/8.2.0/snos/share/m」

$\hookrightarrow$ an:/usr/common/software/man:/usr/common/mss/man:

$\hookrightarrow$ /usr/common/nsg/man:/opt/cray/pe/mpt/7.7.3/gni/m」

$\hookrightarrow$ an/mpich:/opt/cray/pe/atp/2.1.3/man:/opt/cray/al」

$\hookrightarrow$ ps/6.6.43-6.0.7.1_5.45__ga796da32.ari/man:/opt/c 」

$\hookrightarrow \mathrm{ray} / \mathrm{job} / 2.2 .3-6.0 .7 .1 \_5.43 \_$g6c4e934.ari/man:/op」

$\hookrightarrow \quad \mathrm{t} / \mathrm{cray} / \mathrm{pe} / \mathrm{pmi} / 5.0 .14 / \mathrm{man}$ :/opt/cray/pe/libsci/18.

$\hookrightarrow \quad 07.1 / \mathrm{man}$ :/opt/cray/pe/man/csmlversion:/opt/cray/」

$\hookrightarrow$ pe/craype/2.5.15/man:/opt/intel/compilers_and_li 」

$\hookrightarrow$ braries_2019.3.199/linux/man/common:/usr/syscom/」

$\hookrightarrow \mathrm{nsg} / \mathrm{man}$ :/opt/cray/pe/modules/3.2.10.6/share/man: 」

$\hookrightarrow$ /usr/local/man:/usr/share/man:/opt/cray/share/ma」

$\hookrightarrow \mathrm{n}: / \mathrm{opt} / \mathrm{cray} / \mathrm{pe} / \mathrm{man}$ :/opt/cray/share/man:/opt/cray」

$\hookrightarrow$ /share/man

SLURM_JOB_NAME $=$ run $8 \times 8 \times 8 . s h$

XDG_SESSION_ID $=4331$

PE_TRILINOS_DEFAULT_GENCOMPS_CRAY_X86_64 $=87$

PE_TPSL_64_DEFAULT_GENCOMPS_INTEL_interlagos $=160$

PE_PETSC_DEFAULT_GENCOMPILERS_INTEL_mic_knl $=16.0$

PE_FFTW_DEFAULT_TARGET_mic_knl=mic_knl

CRAY_UDREG_INCLUDE_OPTS=-I/opt/cray/udreg/2.3.2-6.0. ।

$\hookrightarrow$ 7.1_5.13__g5196236.ari/include

HOSTNAME $=$ nid05865

SLURM_TOPOLOGY_ADDR=s34.s21.nid05865

SLURMD_NODENAME $=$ nid 05865

PE_TRILINOS_DEFAULT_VOLATILE_PKGCONFIG_PATH=/opt/cra 」

$\hookrightarrow \mathrm{y} / \mathrm{pe} /$ trilinos/12.12.1.1/QPRGENV@/QPE_TRILINOS_DE 」

$\hookrightarrow$ FAULT_GENCOMPS@/@PE_TRILINOS_DEFAULT_TARGET@/lib」

$\hookrightarrow$ /pkgconfig

PE_SMA_DEFAULT_COMPFLAG_GNU=-fcray-pointer

PE_PARALLEL_NETCDF_DEFAULT_VOLATILE_PKGCONFIG_PATH=/ 」

$\hookrightarrow$ opt/cray/pe/parallel-netcdf/1.8.1.3/@PRGENV@/@PE 」

$\hookrightarrow$ _PARALLEL_NETCDF_DEFAULT_GENCOMPSe/lib/pkgconf ig

PE_NETCDF_DEFAULT_VOLATILE_PKGCONFIG_PATH=/opt/cray/ ।

$\hookrightarrow \mathrm{pe} / \mathrm{netcdf} / 4.6 .1 .3 /$ @PRGENV@/@PE_NETCDF_DEFAULT_GE 」

$\hookrightarrow$ NCOMPS@/lib/pkgconfig 
LIBRARYMODULES=acml : alps: cray-dwarf : cray-fftw: cray-g 」 $\hookrightarrow$ a:cray-hdf5:cray-hdf5-parallel:cray-libsci:cray-」

$\hookrightarrow$ libsci_acc:cray-mpich:cray-mpich-abi:cray-mpich2」

$\hookrightarrow \quad$ :cray-netcdf:cray-netcdf-hdf5parallel:cray-paral」

$\hookrightarrow$ lel-netcdf:cray-petsc:cray-petsc-complex:cray-sh」

$\hookrightarrow$ mem:cray-tpsl:cray-trilinos:cudatoolkit:fftw:ga:」

$\hookrightarrow$ hdf5:hdf5-parallel:iobuf:libfast:netcdf:netcdf-h」

$\hookrightarrow \quad$ df5parallel:ntk:onesided:papi:petsc:petsc-comple

$\hookrightarrow \quad \mathrm{x}:$ pmi:tpsl:trilinos:xt-libsci:xt-mpich2:xt-mpt: $\mathrm{x}_{\rfloor}$

$\hookrightarrow \quad \mathrm{t}$-papi

CRAY_SITE_LIST_DIR=/etc/opt/cray/pe/modules

XKEYSYMDB=/usr/X11R6/lib/X11/XKeysymDB

SLURM_PRIO_PROCESS $=0$

RCLOCAL_BASEOPTS=true

INTEL_LICENSE_FILE=28518@crayintel . licenses . nersc . go 」

$\hookrightarrow \quad$ V:28518@intel.licenses.nersc.gov

PE_TPSL_64_DEFAULT_GENCOMPILERS_CRAY_X86_64=8.6

PE_SMA_DEFAULT_COMPFLAG=

PE_MPICH_ALTERNATE_LIBS_dpm=_dpm

PE_HDF5_DEFAULT_GENCOMPILERS_GNU=8.2 $7.16 .1 \quad 5.3 \quad 4.9$

PE_ENV=INTEL

SLURM_NODE_ALIASES $=($ null $)$

PKGCONFIG_ENABLED $=1$

PE_TPSL_DEFAULT_GENCOMPS_CRAY_x86_skylake $=86$

HOST $=$ cori 11

TERM $=x$ term-256color

SHELL=/bin/bash

PE_TPSL_DEFAULT_GENCOMPILERS_GNU_x86_skylake=8.2 7.1

$\hookrightarrow \quad 6.1$

PE_PETSC_DEFAULT_GENCOMPS_CRAY_sandybridge $=86$

INTEL_MINOR_VERSION=19

PROFILEREAD=true

HISTSIZE $=1000$

SLURM_JOB_QOS=regular_1

KMP_HOT_TEAMS_MODE=1

TMPDIR $=/$ tmp

PE_TRILINOS_DEFAULT_VOLATILE_PRGENV=CRAY GNU INTEL

PE_TPSL_DEFAULT_REQUIRED_PRODUCTS=PE_MPICH:PE_LIBSCI

PE_TPSL_DEFAULT_GENCOMPS_GNU_sandybridge=82 $7153 \quad 49$

PE_TPSL_64_DEFAULT_GENCOMPS_INTEL_x86_skylake $=160$

PE_PETSC_DEFAULT_GENCOMPS_INTEL_haswell $1=160$

PE_PETSC_DEFAULT_GENCOMPS_GNU_haswell=71 5349

PE_PARALLEL_NETCDF_DEFAULT_VOLATILE_PRGENV=GNU

PE_NETCDF_DEFAULT_VOLATILE_PRGENV $=G N U$

CRAY_XPMEM_POST_LINK_OPTS=-L/opt/cray/xpmem/2.2.15-6」 $\hookrightarrow \quad$ 0.7.1_5.11_g7549d06.ari/lib64

CRAY_UGNI_POST_LINK_OPTS=-L/opt/cray/ugni / 6.0.14.0-6 」

$\hookrightarrow \quad 0.7 .1$ 3.13__gea11d3d.ari/lib64

CRAYPE_DIR=/opt/cray/pe/craype/2.5.15

SSH_CLIENT $=155.97 .232 .2356043822$

SLURM_TOPOLOGY_ADDR_PATTERN=switch. switch. node

SITE_MODULE_NAMES=darshan

ALT_LINKER=/usr/common/software/altd/2.0/bin/ld

ALTD_SELECT_OFF_USERS=

CRAY_MPICH2_DIR=/opt/cray/pe/mpt/7.7.3/gni/mpich-int 」 $\hookrightarrow \mathrm{el} / 16.0$
PE_PETSC_DEFAULT_GENCOMPS_CRAY_interlagos $=86$

PE_NETCDF_HDF5PARALLEL_DEFAULT_VOLATILE_PKGCONFIG_PA 」

$\hookrightarrow \mathrm{TH}=/ \mathrm{opt} / \mathrm{cray} / \mathrm{pe} / \mathrm{netcdf}$-hdf5parallel/4.6.1.3/@PRG」

$\hookrightarrow$ ENV@/@PE_NETCDF_HDF5PARALLEL_DEFAULT_GENCOMPS@ / ।

$\hookrightarrow$ ib/pkgconfig

PE_HDF5_PARALLEL_DEFAULT_VOLATILE_PKGCONFIG_PATH=/op 」

$\hookrightarrow \quad \mathrm{t} / \mathrm{cray} / \mathrm{pe} / \mathrm{hdf5}$-parallel/1.10.2.0/@PRGENVQ/@PE_HD 」

$\hookrightarrow$ F5_PARALLEL_DEFAULT_GENCOMPS@/lib/pkgconfig

PE_HDF5_DEFAULT_VOLATILE_PRGENV=GNU

PE_FFTW_DEFAULT_VOLATILE_PKGCONFIG_PATH=/opt/cray/pe 」

$\hookrightarrow / \mathrm{fftw} / 3.3 .8 .1 /$ QPE_FFTW_DEFAULT_TARGET@/lib/pkgco 」

$\hookrightarrow$ nfig

LIBRARY_PATH=/opt/intel/compilers_and_libraries_2019」

$\hookrightarrow$.3.199/linux/compiler/lib/intel64:/opt/intel/com」

$\hookrightarrow$ pilers_and_libraries_2019.3.199/linux/mkl/lib/in」

$\hookrightarrow$ tel64

CONDA_SHLVL=1

PYTHON_DIR=/usr/common/software/python/3.6-anaconda- 」

$\hookrightarrow \quad 4.4$

ALTD_SELECT_ON=0

PE_TPSL_DEFAULT_GENCOMPS_CRAY_mic_knl=86

PE_TPSL_64_DEFAULT_GENCOMPILERS_CRAY_interlagos $=8.6$

PE_LIBSCI_DEFAULT_GENCOMPS_GNU_X86_64=71 615149

PE_GA_DEFAULT_VOLATILE_PRGENV=GNU

INTEL_PATH=/opt/intel/compilers_and_libraries_2019.3 」

$\hookrightarrow \quad .199$

CONDA_PROMPT_MODIFIER $=($ stenv $)$

PE_MPICH_GENCOMPS_GNU=71 5149

PE_TPSL_DEFAULT_GENCOMPS_INTEL_X86_64=160

PE_PKGCONFIG_PRODUCTS=PE_MPICH:PE_LIBSCI

PE_MPICH_DEFAULT_GENCOMPILERS_GNU=7.1 5.14 .9

FPATH=: /opt/cray/pe/modules/3.2.10.6/init/sh_funcs/n」

$\hookrightarrow$ o_redirect:/opt/cray/pe/modules/3.2.10.6/init/sh」

$\hookrightarrow$ _funcs/no_redirect:/opt/cray/pe/modules/3.2.10.6 」

$\hookrightarrow /$ init/sh_funcs/no_redirect

MORE $=-s l$

ALTD_VERBOSE $=0$

PE_TPSL_64_DEFAULT_VOLATILE_PKGCONFIG_PATH=/opt/cray 」

$\hookrightarrow / \mathrm{pe} / \mathrm{tpsl} / 18.06 .1 /$ @PRGENV@64/@PE_TPSL_64_DEFAULT_」

$\hookrightarrow$ GENCOMPS@/@PE_TPSL_64_DEFAULT_TARGET@/lib/pkgcon」

$\hookrightarrow$ fig

PE_TPSL_64_DEFAULT_GENCOMPS_CRAY_haswell=86

PE_PETSC_DEFAULT_REQUIRED_PRODUCTS=PE_MPICH:PE_LIBSC 」

$\hookrightarrow \quad I: P E \_H D F 5$ PARALLEL:PE_TPSL

PE_PAPI_DEFAULT_ACCEL_LIBS_nvidia35=,-lcupti , -lcudar 」

$\hookrightarrow \quad \mathrm{t},-$-lcuda

SLURM_SPANK_NERSC_ZONESORT_INTERVAL $=0$

PE_TRILINOS_DEFAULT_GENCOMPILERS_CRAY_X86_64=8.7

PE_CRAY_DEFAULT_FIXED_PKGCONFIG_PATH=/opt/cray/pe/pa 」

$\hookrightarrow$ rallel-netcdf/1.8.1.3/CRAY/8.6/lib/pkgconfig:/op」

$\hookrightarrow$ t/cray/pe/netcdf-hdf5parallel/4.6.1.3/CRAY/8.6/1」

$\hookrightarrow \mathrm{ib} / \mathrm{pkgconfig:/opt/cray/pe/netcdf/4.6.1.3/CRAY/8.}$

$\hookrightarrow$ 6/lib/pkgconfig:/opt/cray/pe/hdf5-parallel/1.10.」

$\hookrightarrow$ 2.0/CRAY/8.6/lib/pkgconfig:/opt/cray/pe/hdf5/1.1

$\hookrightarrow \quad 0.2 .0 / \mathrm{CRAY} / 8.6 / \mathrm{lib} / \mathrm{pkgconfig:/opt/cray/pe/ga/5.3}$ 」

$\hookrightarrow \quad .0 .8 /$ CRAY $/ 8.6 / 1 \mathrm{ib} /$ pkgconfig 
Exploiting Reuse and Vectorization in Blocked Stencil Computations on CPUs and GPUs

PE_FORTRAN_PKGCONFIG_LIBS $=$ mpich $f 90$

PE_TPSL_64_DEFAULT_GENCOMPILERS_CRAY_sandybridge $=8.6$ PE_PETSC_DEFAULT_GENCOMPS_CRAY_X86_64=86

PE_LIBSCI_DEFAULT_OMP_REQUIRES_openmp=_mp

SSH_TTY $=/$ dev $/ p t s / 1$

ALLINEA_QUEUE_DLL=/opt/cray/pe/mpt/7.7.3/gni/mpich-i 」

$\hookrightarrow$ ntel/16.0/lib/libtvmpich.so.3.0.1

PE_SMA_DEFAULT_VOLATILE_PKGCONFIG_PATH=/opt/cray/pe/ ।

$\hookrightarrow \mathrm{mpt} / 7.7 .3$ /gni/sma@PE_SMA_DEFAULT_DIR_DEFAULT64@/ 」

$\hookrightarrow \quad$ lib64/pkgconfig

PYTHONUSERBASE=/global/homes/z/USER/ . local/cori/3.6- 」

$\hookrightarrow \quad$ anaconda-4.4

CRAY_MPICH_BASEDIR=/opt/cray/pe/mpt/7.7.3/gni

PE_TRILINOS_DEFAULT_GENCOMPS_INTEL_X86_64=160

ALPS_APP_ID $=18446744065140005382$

PE_TPSL_64_DEFAULT_GENCOMPS_INTEL_haswell $=160$

PE_TPSL_64_DEFAULT_GENCOMPS_CRAY_X86_skylake $=86$

PE_NETCDF_HDF5PARALLEL_DEFAULT_GENCOMPILERS_GNU=8. 2

$\hookrightarrow \quad 7.16 .1 \quad 5.3 \quad 4.9$

PE_HDF5_PARALLEL_DEFAULT_GENCOMPILERS_GNU=8.2 7.16 .1

$\hookrightarrow \quad 5.34 .9$

JRE_HOME=/usr/lib64/jvm/java/jre

USER=USER

SLURM_NNODES $=1$

PE_TRILINOS_DEFAULT_GENCOMPILERS_INTEL_x $86 \_64=16.0$

PE_TRILINOS_DEFAULT_GENCOMPILERS_GNU_X86_64=8.2 7.3

$\hookrightarrow \quad 5.14 .9$

PE_TPSL_DEFAULT_GENCOMPS_CRAY_X86_64=86

PE_TPSL_64_DEFAULT_GENCOMPILERS_INTEL_mic_knl $=16.0$

PE_PETSC_DEFAULT_GENCOMPILERS_INTEL_interlagos $=16.0$

PE_LIBSCI_DEFAULT_VOLATILE_PRGENV=CRAY GNU INTEL

PE_FFTW_DEFAULT_TARGET_interlagos=interlagos

LS_COLORS $=$ no $=00: \mathrm{fi}=00: \mathrm{di}=01 ; 34: \ln =00 ; 36: \mathrm{pi}=40 ; 33: \mathrm{so}=$ 」

$\hookrightarrow \quad 01 ; 35: \mathrm{do}=01 ; 35: \mathrm{bd}=40 ; 33 ; 01: \mathrm{cd}=40 ; 33 ; 01:$ or $=41 ; 33$;

$\hookrightarrow \quad 01:$ ex $=00 ; 32: * . \mathrm{cmd}=00 ; 32: *$. exe $=01 ; 32: *$.com=01;32: ।

$\hookrightarrow \quad *$.bat $=01 ; 32: *$.btm $=01 ; 32: * . d l l=01 ; 32: *$. ar $=00 ; 31:$ 」

$\hookrightarrow \quad * . \mathrm{tbz}=00 ; 31: * . \operatorname{tg} z=00 ; 31: * . \mathrm{rpm}=00 ; 31: * . \mathrm{deb}=00 ; 31:$ 」

$\hookrightarrow \quad * . \operatorname{arj}=00 ; 31: * . \operatorname{taz}=00 ; 31: * .1 \mathrm{zh}=00 ; 31: * .1 z m a=00 ; 31$ 」

$\hookrightarrow \quad: * . z i p=00 ; 31: * . z 00=00 ; 31: * . z=00 ; 31: * . z=00 ; 31: * . g$ 」

$\hookrightarrow \quad z=00 ; 31: * . b z 2=00 ; 31: * . t b 2=00 ; 31: * . t z 2=00 ; 31: * . t b 」$

$\hookrightarrow \quad z 2=00 ; 31: * . x z=00 ; 31: * . a v i=01 ; 35: * . b m p=01 ; 35: * . f 1$ 」

$\hookrightarrow \quad i=01 ; 35: *$.gif $=01 ; 35: * . j p g=01 ; 35: * . j p e g=01 ; 35: * . m_{」}$

$\hookrightarrow \mathrm{ng}=01 ; 35: * . \mathrm{mov}=01 ; 35: * . \mathrm{mpg}=01 ; 35: * . \mathrm{pcx}=01 ; 35: * . \mathrm{p}$

$\hookrightarrow \mathrm{bm}=01 ; 35: * . \mathrm{pgm}=01 ; 35: * . \mathrm{png}=01 ; 35: * . \mathrm{ppm}=01 ; 35: * . \mathrm{t}$ 」

$\hookrightarrow$ ga $=01 ; 35: *$. tif $=01 ; 35: * . x b m=01 ; 35: * . x p m=01 ; 35: * . d$ 」

$\hookrightarrow \quad l=01 ; 35: * . g l=01 ; 35: * . w m v=01 ; 35: *$. aiff $=00 ; 32: * . a u$ 」

$\hookrightarrow \quad=00 ; 32: * . \mathrm{mid}=00 ; 32: * . \mathrm{mp} 3=00 ; 32: * .0 \mathrm{gg}=00 ; 32: * . \mathrm{voc}$ ।

$\hookrightarrow \quad=00 ; 32: *$ wav $=00 ; 32$ :

LD_LIBRARY_PATH=/opt/gcc/8.2.0/snos/lib64:/opt/cray/」

$\hookrightarrow$ job/2.2.3-6.0.7.1_5.43__g6c4e934.ari/lib64:/opt/」

$\hookrightarrow$ intel/compilers_and_libraries_2019.3.199/linux/c 」

$\hookrightarrow$ ompiler/lib/intel64:/opt/intel/compilers_and_lib」

$\hookrightarrow$ raries_2019.3.199/linux/mkl/lib/intel64:/usr/sys 」

$\hookrightarrow \mathrm{com} / \mathrm{nsg} / \mathrm{lib}$

CSCRATCH=/global/cscratch $1 /$ sd/USER

CSHRCREAD=true
PE_MPICH_FIXED_PRGENV=INTEL

PE_TPSL_64_DEFAULT_GENCOMPILERS_INTEL_haswell $=16.0$ PE_TPSL_64_DEFAULT_GENCOMPILERS_GNU_sandybridge $=8.2$

$\hookrightarrow \quad 7.15 .3 \quad 4.9$

PE_PKGCONFIG_LIBS=darshan-runtime: $\mathrm{mpich}:$ AtpSigHandle 」

$\hookrightarrow \quad r: c r a y-r c a: l i b s c i \_m p i: l i b s c i$

PE_PETSC_DEFAULT_VOLATILE_PRGENV=CRAY CRAY64 GNU

$\hookrightarrow$ GNU64 INTEL INTEL64

PE_LIBSCI_PKGCONFIG_VARIABLES=PE_LIBSCI_OMP_REQUIRES 」

$\hookrightarrow$ _@openmp@:PE_SCI_EXT_LIBPATH:PE_SCI_EXT_LIBNAME

CRAY_RCA_POST_LINK_OPTS=-L/opt/cray/rca/2.2.18-6.0.7 」

$\hookrightarrow \quad$.1_5.47__g2aa4f39.ari/lib64

$\hookrightarrow \quad-$ Irca

PE_TPSL_DEFAULT_GENCOMPS_GNU_haswell=82 715349

PE_PETSC_DEFAULT_GENCOMPS_INTEL_sandybridge $=160$

PE_PETSC_DEFAULT_GENCOMPS_INTEL_interlagos $=160$

PE_PETSC_DEFAULT_GENCOMPS_GNU_sandybridge $=715349$

PE_PETSC_DEFAULT_GENCOMPS_GNU_interlagos $=715349$

PE_PETSC_DEFAULT_GENCOMPILERS_INTEL_skylake $=16.0$

PE_PETSC_DEFAULT_GENCOMPILERS_CRAY_X86_64=8.6

PE_PETSC_DEFAULT_GENCOMPILERS_CRAY_mic_knl $=8.6$

$\mathrm{XNLSPATH}=/ \mathrm{usr} / \mathrm{share} / \mathrm{X} 11 / \mathrm{nls}$

CONDA_EXE=/usr/common/software/python/3.6-anaconda-4 」

$\hookrightarrow .4 / \mathrm{bin} /$ conda

ALTD_ON=1

MPICH_DIR=/opt/cray/pe/mpt/7.7.3/gni/mpich-intel/16.0 PE_TPSL_64_DEFAULT_GENCOMPS_INTEL_sandybridge $=160$

PE_TPSL_64_DEFAULT_GENCOMPILERS_GNU_interlagos $=8.2$

$\hookrightarrow \quad 7.1 \quad 5.3 \quad 4.9$

PE_PETSC_DEFAULT_GENCOMPS_INTEL_mic_knl=160

PE_PETSC_DEFAULT_GENCOMPS_GNU_mic_knl=53

PE_PETSC_DEFAULT_GENCOMPILERS_CRAY_haswell=8.6

PE_PAPI_DEFAULT_PKGCONFIG_VARIABLES=PE_PAPI_ACCEL_LI 」

$\hookrightarrow$ BS_@accelerator@

PE_LIBSCI_DEFAULT_GENCOMPS_CRAY_X86_64=86

MPICH_ABORT_ON_ERROR $=1$

INTEL_VERSION=19.0.3.199

LDAPTLS_REQCERT=never

MPICH_MPIIO_DVS_MAXNODES $=32$

PE_MPICH_FORTRAN_PKGCONFIG_LIBS=mpich 90

PE_TPSL_64_DEFAULT_GENCOMPILERS_CRAY_haswell=8. 6

PE_PETSC_DEFAULT_GENCOMPILERS_INTEL_sandybridge $=16.0$

PE_NETCDF_HDF5PARALLEL_DEFAULT_REQUIRED_PRODUCTS=PE _ 」

$\hookrightarrow$ HDF5_PARALLEL

PE_HDF5_PARALLEL_DEFAULT_REQUIRED_PRODUCTS=PE_MPICH

PE_FFTW_DEFAULT_TARGET_sandybridge=sandybridge

PE_FFTW_DEFAULT_REQUIRED_PRODUCTS=PE_MPICH

CPATH=/opt/intel/compilers_and_libraries_2019.3.199/ 」

$\hookrightarrow \quad$ linux/mkl/include

ATP_POST_LINK_OPTS=-WI, -L/opt/cray/pe/atp/2.1.3/libA 」

$\hookrightarrow \mathrm{pp} /$

HOSTTYPE $=x 86 \_64$

SSH_AUTH_SOCK=/tmp/ssh-6PdT5SIWat/agent. 21653

SLURM_JOBID $=20388358$

RCLOCAL_PRGENV=true

PE_PETSC_DEFAULT_GENCOMPILERS_GNU_mic_knl=5.3 
KMP_HOT_TEAMS_MAX_LEVEL $=2$

GCC_VERSION $=8.2 .0$

PE_TPSL_DEFAULT_GENCOMPS_GNU_interlagos=82 715349

PE_TPSL_DEFAULT_GENCOMPILERS_CRAY_X86_64=8.6

PE_PRODUCT_LIST=CRAYPE_MIC-KNL:CRAY_RCA:CRAY_ALPS:DV」

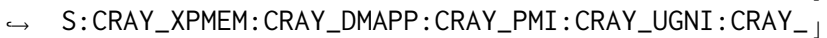

$\hookrightarrow \quad$ UDREG:CRAY_LIBSCI:CRAYPE:INTEL

PE_LIBSCI_GENCOMPS_INTEL_X86_64=160

PE_LIBSCI_DEFAULT_GENCOMPILERS_INTEL_X86_64=16.0

FROM_HEADER=

CRAY_MPICH_ROOTDIR=/opt/cray/pe/mpt/7.7.3

PE_TPSL_DEFAULT_GENCOMPS_GNU_X86_skylake=82 7161

PE_PETSC_DEFAULT_GENCOMPILERS_GNU_X86_64=7.1 5.34 .9

PAGER=less

ALPS_LLI_STATUS_OFFSET $=1$

PE_MPICH_MODULE_NAME=cray-mpich

PE_MPICH_GENCOMPILERS_CRAY $=8.6$

PE_TPSL_64_DEFAULT_GENCOMPILERS_INTEL_X86_64=16.0

PE_PETSC_DEFAULT_GENCOMPS_INTEL_skylake $=160$

PE_PETSC_DEFAULT_GENCOMPS_GNU_skylake $=61$

PE_LIBSCI_GENCOMPILERS_GNU_X86_64=7.1 6.15 .14 .9

CSHEDIT=emacS

ALPS_APP_PE $=0$

PE_TPSL_DEFAULT_GENCOMPS_CRAY_sandybridge $=86$

PE_TPSL_DEFAULT_GENCOMPS_CRAY_haswell $=86$

PE_TPSL_64_DEFAULT_REQUIRED_PRODUCTS=PE_MPICH : PE_LIB 」

$\hookrightarrow \mathrm{SCI}$

PE_MPICH_TARGET_VAR_nvidia20=-lcudart

PE_MPICH_DEFAULT_VOLATILE_PRGENV=CRAY GNU

PE_LIBSCI_GENCOMPS_CRAY_X86_64=86

PE_LIBSCI_DEFAULT_GENCOMPILERS_CRAY_x86_64=8.6

INTEL_MAJOR_VERSION=19

XDG_CONFIG_DIRS=/etc/ $x d g$

PE_TPSL_64_DEFAULT_GENCOMPS_GNU_X86_64=82 715349

PE_TPSL_64_DEFAULT_GENCOMPS_GNU_mic_knl=71 53

PE_PARALLEL_NETCDF_DEFAULT_GENCOMPS_GNU=51 49

PE_NETCDF_DEFAULT_GENCOMPS_GNU=

PE_LIBSCI_PKGCONFIG_LIBS=libsci_mpi : libsci

NLSPATH=/opt/intel/compilers_and_libraries_2019.3.19」

$\hookrightarrow$ 9/1inux/compiler/lib/intel64/locale/\%1_\%t/\%N: /op

$\hookrightarrow \quad$ t/intel/compilers_and_libraries_2019.3.199/linux 」

$\hookrightarrow / \mathrm{mkl} / \mathrm{lib} /$ intel64/locale/\%1_\%t/\%N

DVS_VERSION $=0.9 .0$

CRAY_LIBSCI_DIR=/opt/cray/pe/libsci/18.07.1

CRAY_LIBSCI_BASE_DIR=/opt/cray/pe/libsci/18.07.1

CRAY_DMAPP_INCLUDE_OPTS=-I/opt/cray/dmapp/7.1.1-6.0. 」

$\hookrightarrow$ 7.1_5.45__g5a674e0.ari/include

$\hookrightarrow \quad-$ I/opt/cray/gni-headers/5.0.12.0-6.0.7.1_3.11__g」

$\hookrightarrow \quad 3$ b1768f.ari/include
USERMODULES=PrgEnv-cray:PrgEnv-gnu:PrgEnv-intel:PrgE 」 $\hookrightarrow$ nv-pathscale:PrgEnv-pgi:acml:alps:apprentice:app 」

$\hookrightarrow$ rentice 2 :atp:blcr:cce:chapel:cray-ccdb:cray-fftw」

$\hookrightarrow \quad$ :cray-ga:cray-hdf5:cray-hdf5-parallel:cray-lgdb: 」

$\hookrightarrow$ cray-libsci:cray-libsci_acc:cray-mpich:cray-mpic 」

$\hookrightarrow$ h-compat:cray-mpich2:cray-netcdf:cray-netcdf-hdf」

$\hookrightarrow$ 5parallel:cray-parallel-netcdf:cray-petsc:cray-p」

$\hookrightarrow$ etsc-complex:cray-shmem:cray-snplauncher:cray-tp

$\hookrightarrow$ sl:cray-trilinos:craypat:craype:craypkg-gen:cuda 」

$\hookrightarrow$ toolkit:ddt:fftw:ga:gcc:hdf5:hdf5-parallel:intel」

$\hookrightarrow \quad$ :iobuf:java:lgdb:libfast:libsci_acc:mpich1: netcd 」

$\hookrightarrow \quad f:$ netcdf-hdf5parallel:netcdf-nofsync: netcdf-nofs 」

$\hookrightarrow$ ync-hdf5parallel:ntk:onesided:papi:parallel-netc 」

$\hookrightarrow$ df:pathscale:perftools:perftools-lite:petsc:pets」

$\hookrightarrow \quad$ c-complex:pgi:pmi:stat:totalview:tpsl:trilinos: $x$ 」

$\hookrightarrow$ t-asyncpe: $x t$-craypat:xt-lgdb:xt-libsci:xt-mpich2 」

$\hookrightarrow \quad: x t-m p t: x t-p a p i: x t-s h m e m: x t-t o t a l v i e w$

LIBGL_DEBUG=quiet

MINICOM $=-c$ on

LIBGL_ALWAYS_INDIRECT $=1$

PE_MPICH_GENCOMPILERS_GNU=7.1 5.14 .9

PE_TPSL_DEFAULT_GENCOMPS_CRAY_interlagos $=86$

PE_TPSL_DEFAULT_GENCOMPILERS_GNU_X86_64=8.2 7.15 .3

$\hookrightarrow \quad 4.9$

PE_PKGCONFIG_DEFAULT_PRODUCTS=PE_TRILINOS:PE_TPSL_64 」

$\hookrightarrow \quad: P E \_T P S L: P E \_P E T S C: P E \_P A R A L L E L \_N E T C D F: P E \_N E T C D F \_H$ 」

$\hookrightarrow \quad$ DF5PARALLEL:PE_NETCDF:PE_MPICH:PE_LIBSCI:PE_HDF5」

$\hookrightarrow$ _PARALLEL:PE_HDF5:PE_GA:PE_FFTW2:PE_FFTW

PE_HDF5_DEFAULT_VOLATILE_PKGCONFIG_PATH=/opt/cray/pe 」

$\hookrightarrow$ /hdf5/1.10.2.0/@PRGENV@/@PE_HDF5_DEFAULT_GENCOMP 」

$\hookrightarrow$ S@/lib/pkgconfig

MODULE_VERSION=3.2.10.6

MAIL=/var/mail/USER

$\mathrm{PATH}=/$ global/homes/z/USER/. conda/envs/stenv/bin:/usr 」

$\hookrightarrow$ /common/software/python/3.6-anaconda-4.4/condabi 」

$\hookrightarrow \mathrm{n}: / \mathrm{usr} /$ common/software/bin:/usr/common/mss/bin://

$\hookrightarrow$ usr/common/nsg/bin:/usr/common/software/python/3」

$\hookrightarrow$.6-anaconda-4.4/bin:/usr/common/software/python/」

$\hookrightarrow$ 3.6-anaconda-4.4/1ib/python3.6/site-packages/mpi 」

$\hookrightarrow 4 \mathrm{py} / \mathrm{bin}$ :/opt/gcc/8.2.0/bin:/usr/common/software/」

$\hookrightarrow$ darshan/3.1.4/bin:/usr/common/software/altd/2.0/」

$\hookrightarrow$ bin:/usr/common/software/bin:/usr/common/mss/bin」

$\hookrightarrow \quad:$ /usr/common/nsg/bin:/opt/cray/pe/mpt/7.7.3/gni/」

$\hookrightarrow$ bin:/opt/cray/rca/2.2.18-6.0.7.1_5.47_g2aa4f39.」

$\hookrightarrow$ ari/bin:/opt/cray/alps/6.6.43-6.0.7.1_5.45__ga79」

$\hookrightarrow$ 6da32.ari/sbin:/opt/cray/job/2.2.3-6.0.7.1_5.43_」

$\hookrightarrow$ _g6c4e934.ari/bin:/opt/cray/pe/craype/2.5.15/bin」

$\hookrightarrow \quad$ :/opt/intel/compilers_and_libraries_2019.3.199/1」

$\hookrightarrow$ inux/bin/intel64:/opt/ovis/bin:/opt/ovis/sbin:/u」

$\hookrightarrow$ sr/syscom/nsg/sbin:/usr/syscom/nsg/bin:/opt/cray」

$\hookrightarrow$ /pe/modules/3.2.10.6/bin:/usr/local/bin:/usr/bin」

$\hookrightarrow \quad$ :/bin:/usr/bin/X11:/usr/games:/usr/lib/mit/bin:/」

$\hookrightarrow$ usr/lib/mit/sbin:/opt/cray/pe/bin

SLURM_TASKS_PER_NODE $=1$

PE_TPSL_DEFAULT_GENCOMPILERS_GNU_haswell=8.2 7.15 .3

$\hookrightarrow \quad 4.9$ 
PE_TPSL_64_DEFAULT_GENCOMPILERS_GNU_x86_skylake=8.2 $\hookrightarrow \quad 7.16 .1$

PE_PETSC_DEFAULT_GENCOMPS_CRAY_mic_knl $=86$

PE_PARALLEL_NETCDF_DEFAULT_GENCOMPILERS_GNU=5.1 4.9 PE_NETCDF_DEFAULT_GENCOMPILERS_GNU=8.2 $7.16 .15 .3 \quad 4.9$

PE_FFTW_DEFAULT_TARGET_abudhabi=abudhabi

ATP_IGNORE_SIGTERM $=1$

XTPE_NETWORK_TARGET=aries

$\mathrm{CPU}=\mathrm{x} 86 \_64$

SLURM_WORKING_CLUSTER=cori $:$ ct $11: 6817: 8448$

_=/usr/bin/env

PE_TPSL_64_DEFAULT_GENCOMPILERS_CRAY_x86_skylake $=8.6$

PE_SMA_DEFAULT_DIR_CRAY_DEFAULT $64=64$

PE_NETCDF_HDF5PARALLEL_DEFAULT_GENCOMPS_GNU=

PE_NETCDF_HDF5PARALLEL_DEFAULT_FIXED_PRGENV=CRAY

$\hookrightarrow \quad$ INTEL

PE_HDF5_PARALLEL_DEFAULT_GENCOMPS_GNU=

PE_HDF5_PARALLEL_DEFAULT_FIXED_PRGENV=CRAY INTEL

LDMSD_PLUGIN_LIBPATH=/opt/ovis/lib64/ovis-ldms

JAVA_BINDIR=/usr $/ \mathrm{lib64/jvm/java/bin}$

SLURM_CPUS_PER_TASK $=256$

SLURM_JOB_ID $=20388358$

PMI_NO_FORK $=1$

PE_TPSL_DEFAULT_GENCOMPS_INTEL_interlagos $=160$

PE_TPSL_DEFAULT_GENCOMPILERS_CRAY_mic_knl $=8.6$

PE_TPSL_64_DEFAULT_VOLATILE_PRGENV=CRAY CRAY64 GNU

$\hookrightarrow$ GNU64 INTEL INTEL64

PE_TPSL_64_DEFAULT_GENCOMPS_CRAY_sandybridge $=86$

CRAY_UDREG_POST_LINK_OPTS=-L/opt/cray/udreg/2.3.2-6. 」

$\hookrightarrow \quad 0.7 .1$ 5.13__g5196236.ari/lib64

CONDA_PREFIX=/global/homes/z/USER/.conda/envs/stenv

PE_MPICH_VOLATILE_PRGENV=CRAY GNU

PE_TPSL_DEFAULT_GENCOMPS_GNU_mic_knl $=7153$

CRAY_ALPS_POST_LINK_OPTS=-L/opt/cray/alps/6.6.43-6.0 」

$\hookrightarrow \quad$.7.1_5.45__ga796da32.ari/lib64

CRAYPE_VERSION $=2.5 .15$

INPUTRC $=/$ etc/inputrc

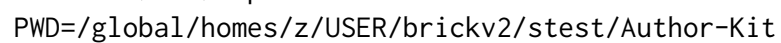

SLURM_JOB_USER=USER

PE_TPSL_DEFAULT_GENCOMPILERS_INTEL_haswell $=16.0$

PE_PETSC_DEFAULT_GENCOMPILERS_GNU_sandybridge $=7.1$

$\hookrightarrow \quad 5.3 \quad 4.9$

PE_MPICH_DEFAULT_GENCOMPS_CRAY $=86$

PE_LIBSCI_DEFAULT_OMP_REQUIRES=
_LMFILES_=/opt/cray/pe/modulefiles/modules/3.2.10.6: 」

$\hookrightarrow /$ /usr/syscom/nsg/modulefiles/nsg/1.2.0:/opt/modul 」

$\hookrightarrow$ efiles/intel/19.0.3.199:/opt/cray/pe/craype/2.5.」

$\hookrightarrow$ 15/modulefiles/craype-network-aries:/opt/cray/pe 」

$\hookrightarrow /$ /modulefiles/craype/2.5.15:/opt/cray/pe/modulefi 」

$\hookrightarrow$ les/cray-libsci/18.07.1:/opt/cray/ari/modulefile」

$\hookrightarrow$ s/udreg/2.3.2-6.0.7.1_5.13__g5196236.ari:/opt/cr 」

$\hookrightarrow \quad$ ay/ari/modulefiles/ugni/6.0.14.0-6.0.7.1_3.13__g」

$\hookrightarrow$ ea11d3d.ari:/opt/cray/pe/modulefiles/pmi/5.0.14: 」

$\hookrightarrow$ /opt/cray/ari/modulefiles/dmapp/7.1.1-6.0.7.1_5.」

$\hookrightarrow$ 45__g5a674e0.ari:/opt/cray/ari/modulefiles/gni-h」

$\hookrightarrow$ eaders/5.0.12.0-6.0.7.1_3.11_g3b1768f.ari:/opt/」

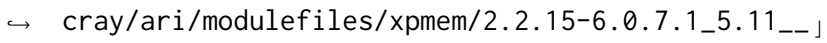

$\hookrightarrow$ g7549d06.ari:/opt/cray/ari/modulefiles/job/2.2.3

$\hookrightarrow-6.0 .7 .1 \_5.43 \_$g6c4e934.ari:/opt/cray/ari/modulef 」

$\hookrightarrow \quad$ iles/dvs/2.7_2.2.118-6.0.7.1_10.1__g58b37a2:/opt 」

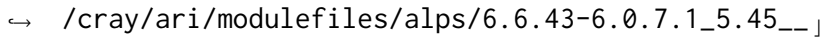

$\hookrightarrow$ ga796da32.ari:/opt/cray/ari/modulefiles/rca/2.2.」

$\hookrightarrow$ 18-6.0.7.1_5.47__g2aa4f39.ari:/opt/cray/pe/modul」

$\hookrightarrow$ efiles/atp/2.1.3:/opt/cray/pe/modulefiles/PrgEnv

$\hookrightarrow-$ intel/6.0.4:/opt/cray/pe/craype/2.5.15/modulefil 」

$\hookrightarrow$ es/craype-mic-knl:/opt/cray/pe/modulefiles/cray-」

$\hookrightarrow$ mpich/7.7.3:/usr/common/software/modulefiles/alt

$\hookrightarrow \mathrm{d} / 2.0: /$ usr/common/software/modulefiles/darshan/3 」

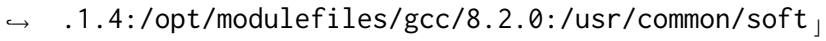

$\hookrightarrow$ ware/modulefiles/python/3.6-anaconda-4.4:/opt/mo 」

$\hookrightarrow$ dulefiles/Base-opts/2.4.135-6.0.7.1_5.6_g718f89」

$\hookrightarrow$ 1.ari

TARGETMODULES=craype-abudhabi : craype-abudhabi-cu: $c r a$ 」

$\hookrightarrow$ ype-accel-host:craype-accel-nvidia20:craype-acce 」

$\hookrightarrow$ 1-nvidia30:craype-accel-nvidia35:craype-barcelon」

$\hookrightarrow$ a:craype-broadwell:craype-haswell:craype-hugepag 」

$\hookrightarrow$ es128K:craype-hugepages128M:craype-hugepages16M: 」

$\hookrightarrow$ craype-hugepages256M:craype-hugepages2M:craype-h」

$\hookrightarrow$ ugepages32M:craype-hugepages4M:craype-hugepages 5 」

$\hookrightarrow \quad 12 \mathrm{~K}:$ craype-hugepages $512 \mathrm{M}:$ craype-hugepages $64 \mathrm{M}: \mathrm{cra}$ ।

$\hookrightarrow$ ype-hugepages8M:craype-intel-knc:craype-interlag」

$\hookrightarrow \quad$ os:craype-interlagos-cu:craype-istanbul:craype-i 」

$\hookrightarrow \quad$ vybridge:craype-mc12:craype-mc8:craype-mic-knl:c」

$\hookrightarrow$ raype-network-aries:craype-network-gemini:craype 」

$\hookrightarrow$-network-infiniband:craype-network-none:craype-ne

$\hookrightarrow$ twork-seastar:craype-sandybridge:craype-shanghai 」

$\hookrightarrow$ :craype-target-compute_node:craype-target-local_」

$\hookrightarrow$ host:craype-target-native:craype-xeon:xtpe-barce」

$\hookrightarrow$ lona:xtpe-interlagos:xtpe-interlagos-cu:xtpe-ist

$\hookrightarrow \quad$ anbul:xtpe-mc12:xtpe-mc8:xtpe-network-gemini:xtp」

$\hookrightarrow$ e-network-seastar:xtpe-shanghai:xtpe-target-nati

$\hookrightarrow \quad$ ve:xtpe-xeon

JAVA_HOME=/usr/lib64/jvm/java

PE_TPSL_DEFAULT_GENCOMPILERS_GNU_mic_knl=7.1 5.3

PE_TPSL_DEFAULT_GENCOMPILERS_CRAY_interlagos $=8.6$

PE_PETSC_DEFAULT_GENCOMPILERS_CRAY_skylake $=8.6$

PE_LIBSCI_MODULE_NAME $=$ cray-libsci $/ 18.07 .1$

PE_INTEL_FIXED_PKGCONFIG_PATH=/opt/cray/pe/mpt/7.7.3 」

$\hookrightarrow$ /gni/mpich-intel/16.0/lib/pkgconfig

PE_TPSL_64_DEFAULT_GENCOMPS_GNU_x86_skylake=82 7161 
PE_MPICH_VOLATILE_PKGCONFIG_PATH=/opt/cray/pe/mpt/7. 」 $\hookrightarrow 7.3 / \mathrm{gni} / \mathrm{mpich}$-@PRGENV@@PE_MPICH_DIR_DEFAULT64@/@ $\hookrightarrow$ PE_MPICH_GENCOMPS@/lib/pkgconfig PE_MPICH_NV_LIBS_nvidia20 $=-$ lcudart PE_LIBSCI_GENCOMPILERS_CRAY_x86_64=8.6

MODULEPATH=/opt/cray/pe/craype/2.5.15/modulefiles: /o 」 $\hookrightarrow \mathrm{pt} / \mathrm{cray} / \mathrm{pe} / \mathrm{modulefiles:/opt/cray/modulefiles:/op}$ $\hookrightarrow$ t/modulefiles:/usr/common/software/modulefiles:/ $\hookrightarrow$ usr/syscom/nsg/modulefiles:/usr/syscom/nsg/opt/m」 $\hookrightarrow$ odulefiles:/usr/common/das/modulefiles:/usr/comm」 $\hookrightarrow$ on/ftg/modulefiles:/opt/cray/craype/default/modu」 $\hookrightarrow$ lefiles:/opt/cray/ari/modulefiles

MAN_POSIXLY_CORRECT $=1$

SHMEM_ABORT_ON_ERROR=1

NSG_HOME=/usr/syscom/nsg

LOADEDMODULES=modules/3.2.10.6:nsg/1.2.0: intel/19.0. ।

$\hookrightarrow \quad 3.199:$ craype-network-aries:craype/2.5.15:cray-li

$\hookrightarrow$ bsci/18.07.1:udreg/2.3.2-6.0.7.1_5.13__g5196236.」

$\hookrightarrow \quad$ ari:ugni/6.0.14.0-6.0.7.1_3.13__gea11d3d.ari:pmi」

$\hookrightarrow \quad / 5.0 .14: \mathrm{dmapp} / 7.1 .1-6.0 .7 .1 \_5.45 \_$_g5a674e0.ari:g」

$\hookrightarrow$ ni-headers/5.0.12.0-6.0.7.1_3.11__g3b1768f.ari:x」

$\hookrightarrow$ pmem/2.2.15-6.0.7.1_5.11__g7549d06.ari:job/2.2.3」

$\hookrightarrow-6.0 .7 .1 \_5.43 \_-g 6 c 4 e 934 . a r i: d v s / 2.7 \_2.2 .118-6.0 .7$ 」

$\hookrightarrow \quad$ 1_10.1__g58b37a2:alps/6.6.43-6.0.7.1_5.45__ga79」

$\hookrightarrow$ 6da32.ari:rca/2.2.18-6.0.7.1_5.47__g2aa4f39.ari:」

$\hookrightarrow$ atp/2.1.3:PrgEnv-intel/6.0.4:craype-mic-knl:cray」

$\hookrightarrow-$ mpich/7.7.3:altd/2.0:darshan/3.1.4:gcc/8.2.0:pyt J

$\hookrightarrow$ hon/3.6-anaconda-4.4:Base-opts/2.4.135-6.0.7.1_5」

$\hookrightarrow \quad$.6_-g718f891.ari

TZ=US/Pacific

SDK_HOME=/usr/lib64/jvm/java

SLURM_JOB_UID $=74457$

PE_TPSL_DEFAULT_GENCOMPILERS_INTEL_mic_knl $=16.0$

PE_TPSL_64_DEFAULT_GENCOMPS_GNU_interlagos $=827153$

$\hookrightarrow \quad 49$

PE_PKG_CONFIG_PATH=/opt/cray/pe/cti $/ 1.0 .7 / \mathrm{lib} / \mathrm{pkgcon}\rfloor$

$\hookrightarrow \mathrm{fig} /$ /opt/cray/pe/cti/1.0.6/lib/pkgconfig

PE_FFTW_DEFAULT_TARGET_x86_skylake=x86_skylake

PE_FFTW_DEFAULT_TARGET_share=share

PE_FFTW_DEFAULT_TARGET_ivybridge=i vybridge

CRAY_DMAPP_POST_LINK_OPTS=-L/opt/cray/dmapp/7.1.1-6. J $\hookrightarrow \quad 0.7 .1 \_5.45 \_$_g5a674e0.ari/lib64

SLURM_NODEID $=0$

PE_TPSL_DEFAULT_GENCOMPILERS_CRAY_x86_skylake $=8.6$

PE_PETSC_DEFAULT_GENCOMPILERS_GNU_skylake $=6.1$

PE_LIBSCI_OMP_REQUIRES_openmp=_mp

CRAY_RCA_INCLUDE_OPTS=-I/opt/cray/rca/2.2.18-6.0.7.1」

$\hookrightarrow \quad$ 5.47__g2aa4f39.ari/include

$\hookrightarrow \quad$-I/opt/cray/krca/2.2.4-6.0.7.1_5.43__g8505b97.ar」

$\hookrightarrow \quad$ i/include

$\hookrightarrow \quad$-I/opt/cray-hss-devel/9.0.0/include

SLURM_SUBMIT_DIR=/global/u1/z/USER/brickv2/stest/Aut 」

$\hookrightarrow$ hor-Kit

PE_MPICH_CXX_PKGCONFIG_LIBS=mpichcxx

CRAY_MPICH_DIR=/opt/cray/pe/mpt/7.7.3/gni/mpich-inte 」

$\hookrightarrow \quad 1 / 16.0$
PE_TPSL_DEFAULT_GENCOMPILERS_INTEL_X86_64=16.0

PE_TPSL_64_DEFAULT_GENCOMPS_CRAY_mic_knl=86

SLURM_TASK_PID $=27877$

PE_MPICH_PKGCONFIG_VARIABLES=PE_MPICH_NV_LIBS_@accel 」

$\hookrightarrow$ erator@:PE_MPICH_ALTERNATE_LIBS_@multithreaded@: ।

$\hookrightarrow$ PE_MPICH_ALTERNATE_LIBS_@dpm@

PE_LIBSCI_DEFAULT_GENCOMPS_INTEL_X86_64=160

SLURM_CPUS_ON_NODE $=256$

PE_MPICH_PKGCONFIG_LIBS=mpich

CRAY_MPICH2_VER=7.7.3

PE_TPSL_DEFAULT_VOLATILE_PKGCONFIG_PATH=/opt/cray/pe 」

$\hookrightarrow$ /tpsl/18.06.1/@PRGENV@/@PE_TPSL_DEFAULT_GENCOMPS ]

$\hookrightarrow$ @/@PE_TPSL_DEFAULT_TARGET@/lib/pkgconfig

PE_TPSL_DEFAULT_GENCOMPILERS_INTEL_X86_skylake $=16.0$

PE_TPSL_64_DEFAULT_GENCOMPILERS_CRAY_mic_knl=8. 6

PE_HDF5_DEFAULT_FIXED_PRGENV=CRAY INTEL

CRAY_PMI_POST_LINK_OPTS=-L/opt/cray/pe/pmi/5.0.14/li 」

$\hookrightarrow$ b64

ENVIRONMENT $=$ BATCH

SLURM_PROCID $=0$

PE_PARALLEL_NETCDF_DEFAULT_FIXED_PRGENV=CRAY INTEL

PE_NETCDF_DEFAULT_FIXED_PRGENV=CRAY INTEL

PE_MPICH_ALTERNATE_LIBS_multithreaded=_mt

PE_LIBSCI_VOLATILE_PKGCONFIG_PATH=/opt/cray/pe/libsc 」

$\hookrightarrow \quad i / 18.07 .1 /$ @PRGENV@/@PE_LIBSCI_GENCOMPS@/@PE_LIBS 」

$\hookrightarrow \quad$ CI_TARGETQ/lib/pkgconfig

PE_GA_DEFAULT_GENCOMPILERS_GNU=5.3 4.9

GPG_TTY=not a tty

SLURM_JOB_NODELIST=nid05865

PE_TPSL_64_DEFAULT_GENCOMPS_GNU_haswell=82 715349

PE_PKGCONFIG_PRODUCTS_DEFAULT=PE_PAPI

PE_NETCDF_HDF5PARALLEL_DEFAULT_VOLATILE_PRGENV=GNU

PE_MPICH_TARGET_VAR_nvidia35=-lcudart

PE_HDF5_PARALLEL_DEFAULT_VOLATILE_PRGENV=GNU

CRAY_LIBSCI_VERSION $=18.07 .1$

QT_SYSTEM_DIR=/usr/share/desktop-data

JDK_HOME=/usr/lib64/jvm/java

SHLVL=3

HOME=/global/homes/z/USER

PE_TPSL_DEFAULT_GENCOMPILERS_INTEL_interlagos $=16.0$

LESS_ADVANCED_PREPROCESSOR=no

OSTYPE=linux

SLURM_LOCALID $=0$

ALTD_PATH=/usr/common/software/altd/2.0

PE_TPSL_DEFAULT_VOLATILE_PRGENV=CRAY CRAY64 GNU GNU64

$\hookrightarrow \quad$ INTEL INTEL64

PE_PETSC_DEFAULT_GENCOMPILERS_CRAY_interlagos $=8.6$

PE_MPICH_DEFAULT_VOLATILE_PKGCONFIG_PATH=/opt/cray/p 」

$\hookrightarrow$ e/mpt/7.7.3/gni/mpich-@PRGENV@@PE_MPICH_DEFAULT_」

$\hookrightarrow$ DIR_DEFAULT64@/@PE_MPICH_DEFAULT_GENCOMPS@/lib/p 」

$\hookrightarrow$ kgconfig

PE_TPSL_DEFAULT_GENCOMPS_INTEL_sandybridge $=160$

PE_TPSL_64_DEFAULT_GENCOMPS_CRAY_interlagos $=86$

CRAY_PMI_INCLUDE_OPTS=-I/opt/cray/pe/pmi/5.0.14/incl 」

$\hookrightarrow$ ude

LS_OPTIONS $=-\mathrm{N}--$ color $=$ none $-\mathrm{T} \theta$ 
Exploiting Reuse and Vectorization in Blocked Stencil Computations on CPUs and GPUs

XCURSOR_THEME $=D M Z$

SLURM_CLUSTER_NAME $=$ cori

SLURM_JOB_CPUS_PER_NODE $=256$

SLURM_JOB_GID $=74457$

GCC_PATH=/opt/gcc/8.2.0

PE_MPICH_DIR_CRAY_DEFAULT $64=64$

PKG_CONFIG_PATH_DEFAULT=/opt/cray/pe/papi/5.6.0.3/li 」

$\hookrightarrow$ b64/pkgconfig

PE_TPSL_DEFAULT_GENCOMPILERS_CRAY_haswell=8.6

ATP_MRNET_COMM_PATH=/opt/cray/pe/atp/2.1.3/1 ibexec/a 」

$\hookrightarrow$ tp_mrnet_commnode_wrapper

CRAYPE_NETWORK_TARGET=aries

WINDOWMANAGER=

PRGENVMODULES=PrgEnv-cray:PrgEnv-gnu:PrgEnv-intel:Pr 」

$\hookrightarrow$ gEnv-pathscale:PrgEnv-pgi

SLURM_SUBMIT_HOST $=$ cori 11

SLURM_GTIDS $=0$

BASH_ENV=/global/homes/z/USER/. bashrc

PE_TPSL_DEFAULT_GENCOMPILERS_INTEL_sandybridge $=16.0$

PE_TPSL_DEFAULT_GENCOMPILERS_GNU_interlagos=8.2 7.1

$\hookrightarrow \quad 5.3 \quad 4.9$

PE_TPSL_64_DEFAULT_GENCOMPILERS_GNU_mic_knl=7.1 5.3

PE_PETSC_DEFAULT_GENCOMPILERS_GNU_haswell=7.1 $5.3 \quad 4.9$

SLURM_JOB_PARTITION=regular

ALTD_SELECT_USERS=

PE_TRILINOS_DEFAULT_REQUIRED_PRODUCTS=PE_MPICH:PE_HD

$\hookrightarrow \quad$ F5_PARALLEL:PE_NETCDF_HDF5PARALLEL:PE_LIBSCI:PE_」

$\hookrightarrow$ TPSL

PE_TPSL_DEFAULT_GENCOMPS_GNU_X86_64=82 715349

PE_TPSL_64_DEFAULT_GENCOMPILERS_INTEL_sandybridge $=16$ 」

$\hookrightarrow .0$

PE_TPSL_64_DEFAULT_GENCOMPILERS_GNU_haswell=8.2 7.1

$\hookrightarrow \quad 5.3 \quad 4.9$

PE_NETCDF_DEFAULT_REQUIRED_PRODUCTS=PE_HDF5

PE_MPICH_NV_LIBS=

PE_HDF5_DEFAULT_GENCOMPS_GNU=

CRAY_LIBSCI_PREFIX_DIR=/opt/cray/pe/libsci/18.07.1/I 」

$\hookrightarrow$ NTEL/16.0/X86_64

CRAY_GNI_HEADERS_INCLUDE_OPTS=-I/opt/cray/gni-header 」

$\hookrightarrow$ s/5.0.12.0-6.0.7.1_3.11_g3b1768f.ari/include

PYTHONPATH=/opt/ovis/lib/python2.7/site-packages

LESS $=-M-I-R$

MACHTYPE $=x 86 \_64-$ suse -1 inux

LOGNAME $=$ USER

CONDA_PYTHON_EXE=/usr/common/software/python/3.6-ana 」

$\hookrightarrow$ conda-4.4/bin/python

PE_MPICH_GENCOMPS_CRAY $=86$

PE_TRILINOS_DEFAULT_GENCOMPS_GNU_X86_64=82 735149

PE_MPICH_DEFAULT_GENCOMPILERS_CRAY $=8.6$

PE_LIBSCI_OMP_REQUIRES=

DMAPP_ABORT_ON_ERROR=1

CVS_RSH=SSh

PE_TPSL_DEFAULT_GENCOMPILERS_CRAY_sandybridge $=8.6$

PE_TPSL_64_DEFAULT_GENCOMPILERS_INTEL_interlagos $=16.0$

PE_MPICH_DEFAULT_GENCOMPS_GNU=71 5149

PE_MPICH_DEFAULT_FIXED_PRGENV=INTEL
PE_LIBSCI_DEFAULT_REQUIRED_PRODUCTS=PE_MPICH

DVS_INCLUDE_OPTS=-I/opt/cray/dvs/2.7_2.2.118-6.0.7.1 」 $\hookrightarrow \quad$ _10.1__g58b37a2/include

XDG_DATA_DIRS=/usr/share

TOOLMODULES=apprentice: apprentice 2 : atp: chapel: $\mathrm{cray}-1$ 」

$\hookrightarrow$ gdb:cray-snplauncher:craypat:craypkg-gen:ddt:gdb」

$\hookrightarrow \quad$ :iobuf:papi:perftools:perftools-lite:stat: totalv

$\hookrightarrow$ iew:xt-craypat:xt-lgdb:xt-papi:xt-totalview

SSH_CONNECTION=155.97.232.235 60438 128.55.209.23 22

SLURM_JOB_ACCOUNT $=$ ACNT

KMP_HW_SUBSET $=64 \mathrm{C}$

PE_TPSL_DEFAULT_GENCOMPILERS_GNU_sandybridge=8.2 7.1

$\hookrightarrow \quad 5.3 \quad 4.9$

PE_LIBSCI_DEFAULT_VOLATILE_PKGCONFIG_PATH=/opt/cray/ 」

$\hookrightarrow$ pe/libsci/18.07.1/@PRGENV@/@PE_LIBSCI_DEFAULT_GE 」

$\hookrightarrow \quad$ NCOMPS@/@PE_LIBSCI_DEFAULT_TARGET@/lib/pkgconfig

PE_GA_DEFAULT_FIXED_PRGENV=CRAY INTEL

PE_FFTW2_DEFAULT_REQUIRED_PRODUCTS=PE_MPICH

CRAY_PRGENVINTEL=loaded

MODULESHOME $=/$ opt $/ \mathrm{cray} / \mathrm{pe} / \mathrm{modules} / 3.2 .10 .6$

SLURM_JOB_NUM_NODES $=1$

PELOCAL_PRGENV=true

PKG_CONFIG_PATH=/usr/common/software/darshan/3.1.4/1 」

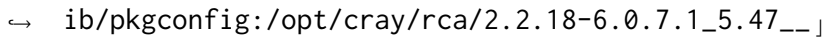

$\hookrightarrow$ g2aa4f39.ari/lib64/pkgconfig:/opt/cray/alps/6.6.」

$\hookrightarrow$ 43-6.0.7.1_5.45__ga796da32.ari/lib64/pkgconfig:/」

$\hookrightarrow \quad$ opt/cray/xpmem/2.2.15-6.0.7.1_5.11__g7549d06.ari 」

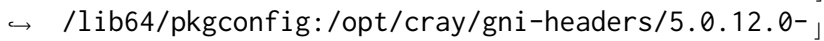

$\hookrightarrow$ 6.0.7.1_3.11__g3b1768f.ari/lib64/pkgconfig:/opt/」

$\hookrightarrow$ cray/dmapp/7.1.1-6.0.7.1_5.45_g5a674e0.ari/lib6」

$\hookrightarrow$ 4/pkgconfig:/opt/cray/pe/pmi/5.0.14/lib64/pkgcon」

$\hookrightarrow \mathrm{fig}$ /opt/cray/ugni/6.0.14.0-6.0.7.1_3.13__gea11d

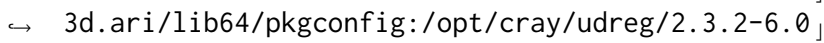

$\hookrightarrow \quad$.7.1_5.13__g5196236.ari/lib64/pkgconfig:/opt/cra 」

$\hookrightarrow$ y/pe/craype/2.5.15/pkg-config:/opt/cray/pe/iobuf 」

$\hookrightarrow$ /2.0.8/lib/pkgconfig:/opt/cray/pe/fftw/2.1.5.9/1

$\hookrightarrow \mathrm{ib} / \mathrm{pkgconfig:/opt/cray/pe/atp/2.1.3/lib/pkgconfig}$

PE_PETSC_DEFAULT_VOLATILE_PKGCONFIG_PATH=/opt/cray/p 」

$\hookrightarrow$ e/petsc/3.8.4.0/complex/@PRGENVQ/@PE_PETSC_DEFAU 」

$\hookrightarrow$ LT_GENCOMPS@/@PE_PETSC_DEFAULT_TARGET@/lib/pkgco 」

$\hookrightarrow$ nfig

PE_MPICH_NV_LIBS_nvidia35=-lcudart

LESSOPEN=lessopen. sh \%s

OMP_PLACES $=$ cores

CONDA_DEFAULT_ENV=stenv

PE_TPSL_64_DEFAULT_GENCOMPS_INTEL_x86_64=160

LIBSCI_BASE_DIR=/opt/cray/pe/libsci/18.07.1

INFOPATH=/opt $/ \mathrm{gcc} / 8.2 .0 / \mathrm{snos} / \mathrm{share} / \mathrm{info}$

PE_TPSL_DEFAULT_GENCOMPS_INTEL_mic_knl=160

PE_TPSL_64_DEFAULT_GENCOMPS_GNU_sandybridge=82 7153

$\hookrightarrow \quad 49$

PE_MPICH_NV_LIBS_nvidia60=-lcudart

PE_LIBSCI_DEFAULT_PKGCONFIG_VARIABLES=PE_LIBSCI_DEFA 」

$\hookrightarrow$ ULT_OMP_REQUIRES_@openmp@:PE_SCI_EXT_LIBPATH:PE $\_」$

$\hookrightarrow$ SCI_EXT_LIBNAME

LIBSCI_VERSION $=18.07 .1$ 
CRAY_CPU_TARGET=mic-knl

PE_TPSL_64_DEFAULT_GENCOMPILERS_GNU_x86_64=8.2 7.1

$\hookrightarrow \quad 5.3 \quad 4.9$

PE_LIBSCI_GENCOMPILERS_INTEL_x86_64=16.0

PE_FFTW_DEFAULT_TARGET_broadwell=broadwell

CRAY_ALPS_INCLUDE_OPTS=-I/opt/cray/alps/6.6.43-6.0.7」

$\hookrightarrow$.1_5.45_ga796da32.ari/include

CRAY_PRE_COMPILE_OPTS=-hnetwork=aries

NERSC_HOST $=$ COri

XDG_RUNTIME_DIR=/run/user $/ 74457$

craype_already_loaded $=0$

PE_TPSL_64_DEFAULT_GENCOMPS_CRAY_x86_64=86

PE_PAPI_DEFAULT_ACCELL_FAMILY_LIBS=

PE_LIBSCI_REQUIRED_PRODUCTS=PE_MPICH

CRAY_XPMEM_INCLUDE_OPTS=-I/opt/cray/xpmem/2.2.15-6.0 」

$\hookrightarrow$.7.1_5.11_g7549d06.ari/include

CRAY_UGNI_INCLUDE_OPTS=-I/opt/cray/ugni/6.0.14.0-6.0 」

$\hookrightarrow \quad$.7.1_3.13_gea11d3d.ari/include

PE_TPSL_DEFAULT_GENCOMPS_INTEL_haswell $=160$

PE_LIBSCI_GENCOMPS_GNU_X86_64=71 615149

PE_LIBSCI_DEFAULT_GENCOMPILERS_GNU_X86_64=7.1 6.15 .1

$\hookrightarrow \quad 4.9$

PE_PETSC_DEFAULT_GENCOMPILERS_INTEL_X86_64=16.0

PE_FFTW_DEFAULT_TARGET_x86_64=x86_64

ATP_HOME $=/$ opt $/ \mathrm{cray} / \mathrm{pe} / \mathrm{atp} / 2.1 .3$

LESSCLOSE=lessclose.sh \%s \%

ALTD_WORKDIR=/global/cscratch1/altd/logs

PE_TPSL_64_DEFAULT_GENCOMPILERS_INTEL_x86_skylake=16 」

$\hookrightarrow .0$

PE_SMA_DEFAULT_DIR_PGI_DEFAULT64 $=64$

PE_PETSC_DEFAULT_GENCOMPILERS_INTEL_haswell=16.0

PE_PETSC_DEFAULT_GENCOMPILERS_GNU_interlagos $=7.15 .3$

$\hookrightarrow \quad 4.9$

PE_PAPI_DEFAULT_ACCEL_LIBS=

PE_INTEL_DEFAULT_FIXED_PKGCONFIG_PATH=/opt/cray $/ \mathrm{pe} / \mathrm{p}$ 」

$\hookrightarrow$ arallel-netcdf/1.8.1.3/INTEL/16.0/1ib/pkgconfig: 」

$\hookrightarrow$ /opt/cray/pe/netcdf-hdf5parallel/4.6.1.3/INTEL/1 」

$\hookrightarrow$ 6.0/lib/pkgconfig:/opt/cray/pe/netcdf/4.6.1.3/IN J

$\hookrightarrow \mathrm{TEL} / 16.0 / \mathrm{ib} / \mathrm{pkgconfig:/opt/cray/pe/mpt/7.7.3/gn」}$

$\hookrightarrow \quad \mathrm{i} / \mathrm{mpich}-i n t e l / 16.0 / \mathrm{lib} / \mathrm{pkgconfig:/opt/cray/pe/hd}$

$\hookrightarrow$ f5-parallel/1.10.2.0/INTEL/16.0/lib/pkgconfig:/o」

$\hookrightarrow \mathrm{pt} / \mathrm{cray} / \mathrm{pe} / \mathrm{hdf5} / 1.10 .2 .0 / \mathrm{INTEL} / 16.0 / \mathrm{ib} / \mathrm{pkgconfi}$ ।

$\hookrightarrow \mathrm{g}: / \mathrm{opt} / \mathrm{cray} / \mathrm{pe} / \mathrm{ga} / 5.3 .0 .8 / \mathrm{INTEL}_{1} 18.0 / \mathrm{ib} / \mathrm{pkgconf}$ 」

$\hookrightarrow$ ig

PE_GA_DEFAULT_VOLATILE_PKGCONFIG_PATH=/opt/cray $/ \mathrm{pe} / \mathrm{g}$ 」

$\hookrightarrow a / 5.3 .0 .8 /$ /QPRGENV@/QPE_GA_DEFAULT_GENCOMPS@/lib/」

$\hookrightarrow$ pkgconfig

PE_GA_DEFAULT_GENCOMPS_GNU=53 49

PE_FFTW_DEFAULT_TARGET_haswell=haswell
CRAY_LD_LIBRARY_PATH=/usr/common/software/darshan/3. . $\hookrightarrow$ 1.4/lib:/opt/cray/pe/mpt/7.7.3/gni/mpich-intel/1

$\hookrightarrow$ 6.0/lib:/opt/cray/rca/2.2.18-6.0.7.1_5.47__g2aa4」

$\hookrightarrow$ f39.ari/lib64:/opt/cray/alps/6.6.43-6.0.7.1_5.45」

$\hookrightarrow$ _-ga796da32.ari/lib64:/opt/cray/xpmem/2.2.15-6.0」

$\hookrightarrow \quad$.7.1_5.11__g7549d06.ari/lib64:/opt/cray/dmapp/7.」

$\hookrightarrow$ 1.1-6.0.7.1_5.45__g5a674e0.ari/lib64:/opt/cray/p

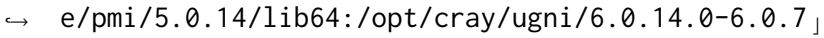

$\hookrightarrow$.1_3.13__gea11d3d.ari/lib64:/opt/cray/udreg/2.3. 」

$\hookrightarrow$ 2-6.0.7.1_5.13__g5196236.ari/lib64:/opt/cray/pe/」

$\hookrightarrow \quad$ libsci/18.07.1/INTEL/16.0/x86_64/1ib

G_BROKEN_FILENAMES $=1$

SCRATCH=/global/cscratch1/sd/USER

SLURM_MEM_PER_NODE $=89088$

intel_already_loaded $=0$

PE_PETSC_DEFAULT_GENCOMPS_INTEL_x86_64=160

PE_PETSC_DEFAULT_GENCOMPS_GNU_X86_64=71 5349

PE_PETSC_DEFAULT_GENCOMPS_CRAY_haswell $=86$

PE_MPICH_DEFAULT_DIR_CRAY_DEFAULT $64=64$

JAVA_ROOT=/usr/lib64/jvm/java

COLORTERM $=1$

BASH_FUNC_module $\%=()$ \{ eval

$\hookrightarrow$ Y /opt/cray/pe/modules/3.2.10.6/bin/modulecmd

$\hookrightarrow$ bash $\$ *^{\prime}$

\}

+ 1sb_release -a

LSB Version:

Distributor ID:

$\mathrm{n} / \mathrm{a}$

Description:

SUSE

$\hookrightarrow \quad \mathrm{SP} 3$

Release: $\quad 12.3$

Codename: $\mathrm{n} / \mathrm{a}$

+ uname -a

Linux nid05865 4.4.103-6.38_4.0.153-cray_ari_c \#1 SMP

$\hookrightarrow$ Thu Nov 1 16:05:05 UTC 2018 (6ef8fef) x86_64

$\hookrightarrow \quad$ x86_64 x86_64 GNU/Linux

+1 scpu

Architecture: $\quad$ x86_64

CPU op-mode(s): $\quad$ 32-bit, 64-bit

Byte Order: Little Endian

CPU(s): $\quad 272$

On-line CPU(s) list: $0-271$

Thread(s) per core: 4

Core(s) per socket: 68

Socket (s): 1

NUMA node(s): $\quad 1$

Vendor ID: GenuineIntel

CPU family: 6

Model: $\quad 87$

Model name: Intel(R) Xeon Phi(TM) CPU 7250

$\hookrightarrow$ @ $1.40 \mathrm{GHz}$

Stepping:

CPU MHz:

CPU max MHz:

$\mathrm{CPU}$ min $\mathrm{MHz}$ :

BogoMIPS:
1

1401.000

1401.0000

1000.0000

2799.85 
Exploiting Reuse and Vectorization in Blocked Stencil Computations on CPUs and GPUs

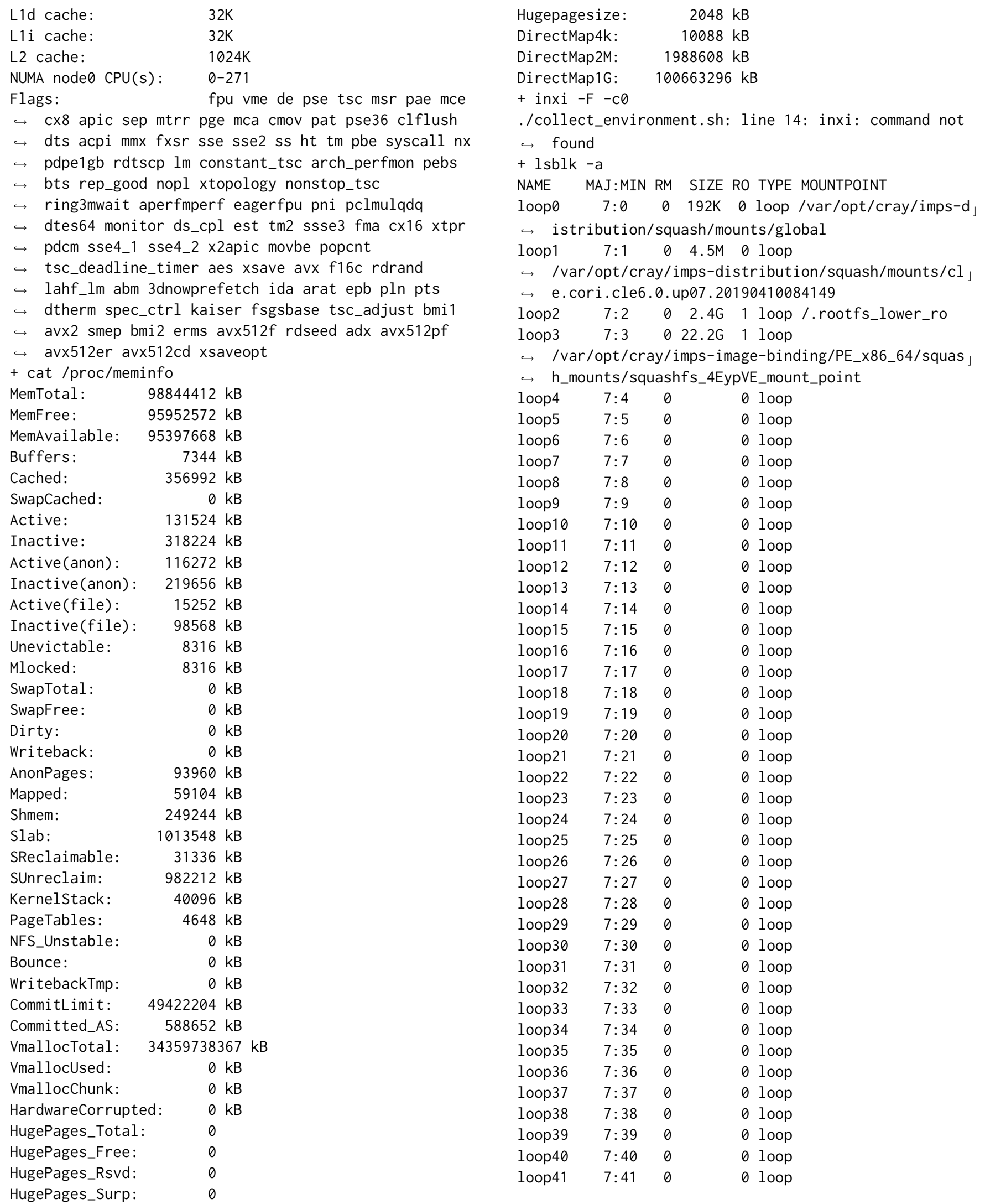




\begin{tabular}{|c|c|c|c|}
\hline loop42 & $7: 42$ & 0 & 0 loop \\
\hline loop43 & $7: 43$ & 0 & 0 loop \\
\hline loop44 & $7: 44$ & 0 & 0 loop \\
\hline loop45 & $7: 45$ & 0 & 0 loop \\
\hline loop46 & $7: 46$ & 0 & 0 loop \\
\hline loop47 & $7: 47$ & 0 & 0 loop \\
\hline loop48 & $7: 48$ & 0 & 0 loop \\
\hline loop49 & $7: 49$ & 0 & 0 loop \\
\hline loop50 & $7: 50$ & 0 & 0 loop \\
\hline loop51 & $7: 51$ & 0 & 0 loop \\
\hline loop52 & $7: 52$ & 0 & 0 loop \\
\hline loop53 & $7: 53$ & 0 & 0 loop \\
\hline loop54 & $7: 54$ & 0 & 0 loop \\
\hline loop55 & $7: 55$ & 0 & 0 loop \\
\hline loop56 & $7: 56$ & 0 & 0 loop \\
\hline loop57 & $7: 57$ & 0 & 0 loop \\
\hline loop58 & $7: 58$ & 0 & 0 loop \\
\hline loop59 & $7: 59$ & 0 & 0 loop \\
\hline loop60 & $7: 60$ & 0 & 0 loop \\
\hline loop61 & $7: 61$ & 0 & 0 loop \\
\hline loop62 & $7: 62$ & 0 & 0 loop \\
\hline loop63 & $7: 63$ & 0 & 0 loop \\
\hline loop64 & $7: 64$ & 0 & 0 loop \\
\hline loop65 & $7: 65$ & 0 & 0 loop \\
\hline loop66 & $7: 66$ & 0 & 0 loop \\
\hline loop67 & $7: 67$ & 0 & 0 loop \\
\hline loop68 & $7: 68$ & 0 & 0 loop \\
\hline loop69 & $7: 69$ & 0 & 0 loop \\
\hline loop70 & $7: 70$ & 0 & 0 loop \\
\hline loop71 & $7: 71$ & 0 & 0 loop \\
\hline loop72 & $7: 72$ & 0 & 0 loop \\
\hline loop73 & $7: 73$ & 0 & 0 loop \\
\hline loop74 & $7: 74$ & 0 & 0 loop \\
\hline loop75 & $7: 75$ & 0 & 0 loop \\
\hline loop76 & $7: 76$ & 0 & 0 loop \\
\hline loop77 & $7: 77$ & 0 & 0 loop \\
\hline loop78 & $7: 78$ & 0 & 0 loop \\
\hline loop79 & $7: 79$ & 0 & 0 loop \\
\hline loop80 & $7: 80$ & 0 & 0 loop \\
\hline loop81 & $7: 81$ & 0 & 0 loop \\
\hline loop82 & $7: 82$ & 0 & 0 loop \\
\hline loop83 & $7: 83$ & 0 & 0 loop \\
\hline loop84 & $7: 84$ & 0 & 0 loop \\
\hline loop85 & $7: 85$ & 0 & 0 loop \\
\hline loop86 & $7: 86$ & 0 & 0 loop \\
\hline loop87 & $7: 87$ & 0 & 0 loop \\
\hline loop88 & $7: 88$ & 0 & 0 loop \\
\hline loop89 & $7: 89$ & 0 & 0 loop \\
\hline loop90 & $7: 90$ & 0 & 0 loop \\
\hline loop91 & $7: 91$ & 0 & 0 loop \\
\hline loop92 & $7: 92$ & 0 & 0 loop \\
\hline loop93 & $7: 93$ & 0 & 0 loop \\
\hline loop94 & $7: 94$ & 0 & 0 loop \\
\hline loop95 & $7: 95$ & 0 & 0 loop \\
\hline loop96 & $7: 96$ & 0 & 0 loop \\
\hline loop97 & $7: 97$ & 0 & 0 loop \\
\hline loop98 & $7: 98$ & 0 & 0 loop \\
\hline
\end{tabular}

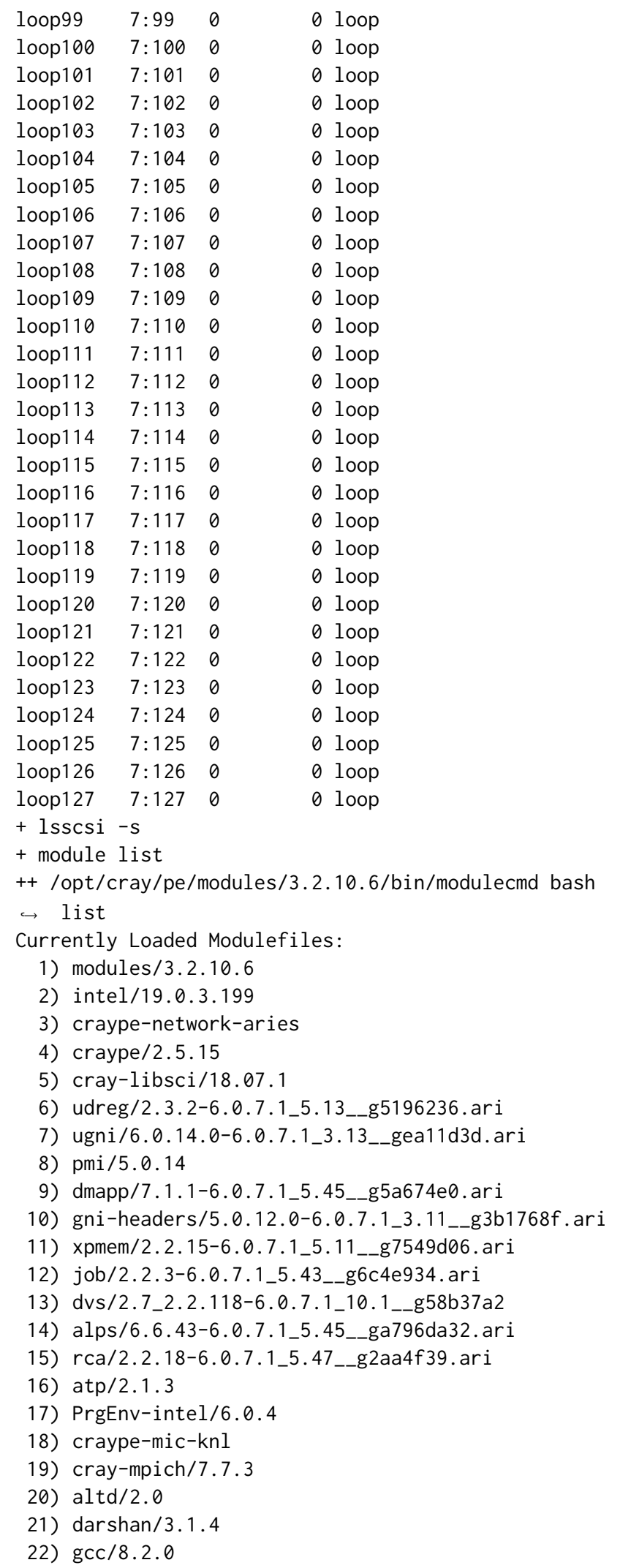


23) python/3.6-anaconda-4.4

24) Base-opts/2.4.135-6.0.7.1_5.6_-g718f891.ari

+ eval

+ nvidia-smi

NVIDIA-SMI has failed because it couldn't communicate

$\hookrightarrow$ with the NVIDIA driver. Make sure that the latest

$\hookrightarrow$ NVIDIA driver is installed and running.

+1 shw -short -quiet -sanitize

+ cat

./collect_environment.sh: line 19: lshw: command not

$\hookrightarrow$ found

+1 spci

./collect_environment.sh: line 19: lspci: command not

$\hookrightarrow$ found

\section{ARTIFACT EVALUATION}

Verification and validation studies: We applied our approach on synthetic stencils with varying shape and order to expose how these factors affect the performance of our approach.

We also gathered hardware profiling data and looked at the generated assembly to identify the differences between our code and the baseline. We performed detailed analysis based on these results.

The results from these analysis agree with the theory we presented in the paper.

Accuracy and precision of timings: On KNL and Skylake-X, for all the stencil variants we drop the first iteration due to cold start, and time the stencils kernel running consecutively until total time exceeds 5 seconds to reduce variation between each stencil iterations. This is enough time for taking the average of at least 25 iterations with the code generated using our approach. Time is taken using omp_wtime(). The throughput is calculated based on the average time taken for one iteration.

On P100, each stencil is run one iteration untimed due to cold start and then timed for 100 iterations using cudaEvent_t. The throughput is calculated based on the average time taken for one iteration.

Used manufactured solutions or spectral properties: Not applicable

Quantified the sensitivity of results to initial conditions and/or parameters of the computational environment: No

Controls, statistics, or other steps taken to make the measurements and analyses robust to variability and unknowns in the system. No 